Bull. Soc. math. France

131 (1), 2003, p. 59-121

\title{
CLASSES DE CHERN ET CLASSES DE CYCLES EN COHOMOLOGIE RIGIDE
}

\author{
PAR Denis Petrequin
}

\begin{abstract}
RÉSUmÉ. - Nous construisons dans cet article les classes de Chern et les classes de cycles en cohomologie rigide. Nous démontrons par la suite que ces constructions vérifient bien les propriétés attendues. La cohomologie rigide est donc une cohomologie de Weil.

ABStRACT (Chern classes and cycle classes in rigid cohomology)

We define in this article Chern classes and cycle classes in rigid cohomology. Then we prove that these constructions verify the expected properties. The rigid cohomology is a Weil cohomology.
\end{abstract}

\section{Table des matières}

Introduction 60

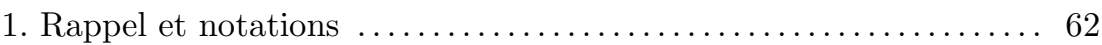

2. Homologie rigide - Formalisme de Bloch-Ogus . ............ 66

3. Classes de cohomologie associées à un pseudo-diviseur . . . . . . 72

4. Classes de Chern ................................... 85

5. Comparaison avec la cohomologie cristalline et additivité ...... 88

6. Comparaison avec le morphisme de Gysin - Classes de cycles . . . 104

7. Intersection de cycles et applications .................... 112

Bibliographie ........................................ 119

Texte reçu le 25 septembre 2001, accepté le 20 mars 2002

Denis Petrequin, IRMAR, Campus de Beaulieu, 35042 Rennes Cedex (France)

E-mail : petrus@maths.univ-rennes1.fr • Url : www.maths.univ-rennes1.fr/ petrus Classification mathématique par sujets (2000). — 14F30.

Mots clefs. - Cohomologie rigide, cohomologie cristalline, classes de cycles, classes de Chern. Cet article a bénéficié du support des réseaux européens TMR (n ERBFMRXCT-960006) et IHP (no HPRN-CT2000-00120). 


\section{Introduction}

La cohomologie rigide, introduite par Berthelot [2], est une théorie cohomologique $p$-adique : c'est une généralisation de la cohomologie de MonskyWashnitzer [26] et de la cohomologie cristalline [1]. Berthelot a démontré qu'elle vérifiait la propriété de finitude pour les variétés lisses ou propres [7], ainsi que la dualité de Poincaré et la formule de Künneth [6] ; le théorème de finitude a ensuite été étendu aux variétés quelconques par Grosse-Klönne [17]. Dans cet article, nous étudions les classes de Chern et les classes de cycles. Nous obtenons que la cohomologie rigide satisfait à tous les axiomes des cohomologies de Weil.

La cohomologie rigide est la cohomologie du complexe de de Rham surconvergent du tube d'une compactification de la variété. La première classe de Chern d'un faisceau inversible est construite à l'aide de cocycles de Čech à valeur dans ce complexe de de Rham surconvergent. Ce cocycle est calculé à l'aide de relèvements d'un cocycle représentant le faisceau inversible dont on est parti. On peut alors construire les classes de Chern de manière classique en utilisant [18]. Cependant, on ne dispose pas en cohomologie rigide de morphismes d'image directe en toute généralité. On ne peut donc pas directement utiliser la méthode de [18] pour démontrer l'additivité des classes de Chern définies précédemment. On veut utiliser la méthode qui consiste à se ramener à un cas universel en considérant un topos classifiant [8]. Cependant, il semble difficile de définir la cohomologie rigide d'un topos classifiant en utilisant la définition basée sur des plongements. Après s'être ramené au cas des variétés propres, nous utiliserons une définition de la cohomologie rigide basée sur les topos cristallin de niveau $m$. Nous réinterprétons alors le calcul à base de cocycle précédant de manière cristalline ce qui permet de le généraliser au cas des variétés simpliciales. On achève la démonstration de l'additivité en suivant [8]. Nous en profitons pour construire des classes de Chern à valeurs dans la cohomologie cristalline de niveau $m$. Pour les classes de cycles, le morphisme trace peut-être vu comme un élément de l'homologie rigide - dual de la cohomologie rigide à support compact. On appelle cet élément la classe fondamentale. Les classes de cycles se définissent alors par fonctorialité et linéarité. On montre alors que cela correspond à l'image de 1 par le morphisme de Gysin, ce qui permet de prouver que si $D$ est un diviseur d'une variété lisse, la classe ainsi définie est égale à la classe du pseudo-diviseur associé à $D$. On en déduit la compatibilité des classes de cycles à l'équivalence rationnelle.

Précisons les différentes parties de l'article.

Nous commencerons par un chapitre préliminaire, dans lequel on trouvera des rappels sur la définition de la cohomologie rigide ainsi que sur ses propriétés. Nous en profiterons pour faire aussi quelques rappels sur les cycles et leurs intersections, en particulier pour les variétés singulières. 
Dans la deuxième partie, nous définirons l'homologie rigide (les classes de cycles se définissent naturellement dans l'homologie et non la cohomologie si on s'intéresse à des variétés singulières). Nous montrerons alors comment on peut, à partir des propriétés de la cohomologie rigide, montrer que l'on a un formalisme de théorie de dualité de Poincaré au sens de Bloch-Ogus [10] : nous exhiberons la construction de la classe fondamentale d'une variété intègre qui sera la base de la définition des classes de cycles. Toutes les constructions de cette partie sont formelles.

Dans la troisième partie, nous construirons, par un calcul de cocycles, la classe de cohomologie associée à un pseudo-diviseur. On commencera par traiter le cas des variétés propres pour s'intéresser par la suite aux variétés ouvertes. C'est cette construction qui est le cour de cet article.

Dans la partie 4, nous définirons la première classe de Chern d'un fibré inversible comme un cas particulier de la classe de cohomologie d'un pseudodiviseur. Nous construirons alors les classes de Chern en suivant la méthode de Grothendieck [20]. Pour ce faire nous aurons besoin du calcul de la cohomologie rigide d'un fibré projectif relatif. Les classes de Chern ainsi construites sont fonctorielles et normalisées. Cependant on ne peut pas appliquer directement les méthodes classiques pour démontrer l'additivité des classes de Chern.

Dans la cinquième partie, nous réinterpréterons, pour les variétés propres, la cohomologie rigide comme une cohomologie cristalline limite. Précisément nous introduirons le topos limite inductive des topos cristallins de niveau $m$. Dans ce dernier nous pouvons définir des classes de Chern et vérifier l'additivité. Un théorème de comparaison avec le cas rigide nous permet alors de finir l'étude des classes de Chern rigides. Nous définirons aussi les classes de Chern dans chaque topos cristallin de niveau $m$.

Pour finir, nous démontrerons une comparaison entre la classe d'un pseudodiviseur et la classe d'un cycle. Cela nous permettra alors de montrer que les classes de cycles sont compatibles à l'équivalence rationnelle. On peut alors montrer la compatibilité des classes de cycles aux intersections. On conclura en énonçant un théorème de Riemann-Roch, la formule de self-intersection, le calcul de la cohomologie d'un éclaté et de l'action du Frobenius sur les classes de cycles. Ces propriétés se déduisent des théorèmes analogues sur les groupes de Chow.

Cet article est tiré de ma thèse de doctorat [30].

Remerciements. - Je tiens à remercier P. BERTHELOT pour tous les conseils qu'il m'a prodigués tout au long de ma thèse et de la rédaction de cet article. Je tiens aussi à remercier le rapporteur qui m'a indiqué la notion de pseudodiviseur permettant d'unifier et de clarifier les constructions traitées dans cet article. 
Notation. - Tout au long de cet article, $k$ désignera un corps de caractéristique $p>0$. On appelera $k$-variété un schéma séparé de type fini sur $\operatorname{Spec}(k)$.

\section{Rappel et notations}

1.1. Cohomologie rigide. - Cette partie rappelle les définitions et les propriétés de la cohomologie rigide. Tout ce qui s'y trouve est issu des articles de Berthelot sur le sujet [2], [4], [6], [7].

Rappelons pour commencer la construction de la cohomologie rigide [7]. Soient $X$ une $k$-variété, $Z$ un sous-schéma fermé de $X$ et $U=X-Z$. Il existe d'après Nagata [27], une variété propre $\bar{X}$ et une immersion ouverte $j_{X}: X \hookrightarrow \bar{X}$ (on notera $j_{U}: U \hookrightarrow \bar{X}$ ). Soit $\mathscr{V}$ un anneau de valuation discrète, de corps résiduel $k$ et de corps des fractions $K$. On suppose alors qu'il existe une immersion fermée $\bar{X} \hookrightarrow \mathscr{Y}$ dans un schéma formel $\mathscr{Y} \operatorname{sur} \operatorname{Spf}(\mathscr{V})$ lisse au voisinage de $X$ - cette condition technique peut être supprimée en utilisant des résolutions de Čech, nous la garderons pour simplifier le propos. On considère la fibre générique rigide $\mathrm{Y}$ de $\mathscr{Y}[32]$ et on note $\mathrm{sp}: \mathrm{Y} \rightarrow \mathscr{Y}$ le morphisme de spécialisation. Rappelons $[4,1.1]$ si on note $\mathscr{Y}_{0}$ la réduction de $\mathscr{Y}$ sur $k$, on appelle, pour tout sous- $k$-schéma $T$ de $\mathscr{Y} 0$, tube de $T$ et on note $] T$ [ le sous-ensemble $\mathrm{sp}^{-1}(T)$ des points de $\mathrm{Y}$ qui se spécialisent dans $T$.

Avec les notations précédentes, on appelle voisinage strict $[4,1.2]$ de $] X[$ dans $] \bar{X}[$, tout ouvert $V$ de $] \bar{X}[$ tel que le recouvrement $(V,] \bar{X}-X[)$ soit admissible. Dès lors, pour tout faisceau $\mathscr{E}$ sur $] \bar{X}[$, on note

$$
j^{\dagger} \mathscr{E}:=\lim _{V} \alpha_{V *} \alpha_{V}^{*} \mathscr{E},
$$

où la limite inductive est prise sur tous les voisinages stricts de $] X[$ dans $] \bar{X}[$ et $\alpha_{V}$ désigne l'inclusion $\left.V \hookrightarrow\right] \bar{X}$ [. On définit de même $j_{U}^{\dagger}$.

On regarde alors le complexe simple associé au complexe double

$$
\left(j_{X}^{\dagger} \Omega_{] \bar{X}[}^{\star} \longrightarrow j_{U}^{\dagger} \Omega_{] \bar{X}[}^{\star}\right)_{s}
$$

où le terme de bidegré $(0,0)$ est $j_{X}^{\dagger} \mathscr{O}_{] \bar{X}}$. La différentielle

$$
\mathrm{d}: j_{X}^{\dagger} \Omega_{] \bar{X}[}^{i} \oplus j_{U}^{\dagger} \Omega_{] \bar{X}[}^{i-1} \longrightarrow j_{X}^{\dagger} \Omega_{] \bar{X}[}^{i+1} \oplus j_{U}^{\dagger} \Omega_{] \bar{X}[}^{i}
$$

est donnée par

$$
\left(\begin{array}{cc}
\mathrm{d}_{X} & 0 \\
r & -\mathrm{d}_{U}
\end{array}\right)
$$

où $r$ est la restriction et

$$
\mathrm{d}_{X}: j_{X}^{\dagger} \Omega_{] \bar{X}[}^{i-1} \longrightarrow j_{X}^{\dagger} \Omega_{] \bar{X}[}^{i} \quad\left(\text { resp. } \mathrm{d}_{U}: j_{U}^{\dagger} \Omega_{] \bar{X}[}^{i-1} \longrightarrow j_{U}^{\dagger} \Omega_{] \bar{X}[}^{i}\right)
$$

TOME $131-2003-\mathrm{N}^{\mathrm{O}} 1$ 
est obtenue en appliquant à d le foncteur $j_{X}^{\dagger}\left(\right.$ resp. $\left.j_{U}^{\dagger}\right)$. Berthelot [7] montre alors que les groupes de cohomologie

$$
\mathbb{H}^{i}(] \bar{X}\left[,\left(j_{X}^{\dagger} \Omega_{] X[}^{\star} \rightarrow j_{U}^{\dagger} \Omega_{] X[}^{\star}\right)_{s}\right)
$$

ne dépendent pas des choix faits.

On définit alors les groupes de cohomologie rigide de $X$ à support dans $Z$ par

$$
H_{Z, \mathrm{rig}}^{i}(X / K):=\mathbb{H}^{i}(] \bar{X}\left[,\left(j_{X}^{\dagger} \Omega_{] \bar{X}[}^{\star} \rightarrow j_{U}^{\dagger} \Omega_{] \bar{X}[}^{\star}\right)_{s}\right) .
$$

En prenant $Z=X$, on trouve la cohomologie rigide

$$
H_{\text {rig }}^{i}(X / K):=\mathbb{H}^{i}(] \bar{X}\left[, j_{X}^{\dagger} \Omega_{] \bar{X}}^{\star}\right) .
$$

Notation. - Nous noterons

$$
\mathbb{R} \underline{\underline{\Gamma}}_{\text {rig }}((X, \bar{X}) / K):=\mathbb{R s p}_{*} j_{X}^{\dagger} \Omega_{] \bar{X}}^{\star}[
$$

vu comme élément de la catégorie dérivée des $K$-vectoriels sur $\bar{X}$. Par abus, nous omettrons le $\bar{X}$ dans la notation ci-dessus.

Énonçons quelques propriétés classiques. Si on se donne $X$ (resp. $Y$ ), $Z$ un fermé de $X$ (resp. $T$ un fermé de $Y$ ) et un morphisme $f: X \rightarrow Y$ tel que $f^{-1}(T) \subset Z$, il existe un morphisme de fonctorialité contravariante

$$
f^{*}: H_{T, \mathrm{rig}}^{i}(Y / K) \longrightarrow H_{Z, \mathrm{rig}}^{i}(X / K) .
$$

Il existe aussi un cup-produit

$$
H_{\text {rig }}^{i}(X) \otimes H_{Z, \text { rig }}^{j}(X) \stackrel{\cup}{\longrightarrow} H_{Z, \text { rig }}^{i+j}(X) .
$$

De plus les morphismes de fonctorialités sont compatibles aux cup-produits.

Il est aussi direct de vérifier que la cohomologie d'une somme disjointe est la somme directe des cohomologies.

Il existe aussi (voir [2]) une notion de cohomologie rigide à support compact, notée $H_{c, \text { rig }}^{*}(X / K)$, qui est covariante par rapport aux immersions ouvertes et contravariante par rapport aux morphismes propres.

Rappelons maintenant les principales propriétés de la cohomologie rigide. On trouvera les démonstrations dans [7], [6] et [17].

ThÉORÈme 1.1 (Berthelot). - Avec les notations précédentes, on a les propriétés suivantes :

- Pour tout $k$-variété $X, H_{\mathrm{rig}}^{i}(X / K)$ et $H_{c, \mathrm{rig}}^{i}(X / K)$ sont de dimension finie.

- Si $X$ est équidimensionnelle de dimension $n$,

$$
H_{\text {rig }}^{i}(X / K)=0 \quad \text { et } \quad H_{c, \text { rig }}^{i}(X / K)=0 \text { pour } i \notin[0,2 n] .
$$

- Si on suppose que $X$ est lisse et si $Z$ est un sous-schéma fermé de codimension $r$, on a pour tout $i<2 r$

$$
H_{Z, \text { rig }}^{i}(X / K)=0 .
$$

BULLETIN DE LA SOCIÉtÉ MATHÉMATIQUE DE FRANCE 
- Il existe une application trace canonique $H_{c, \text { rig }}^{2 n}(X / K) \rightarrow K$ qui, composée avec la multiplication, induit des applications

$$
H_{Z, \text { rig }}^{i}(X / K) \times H_{c, \text { rig }}^{2 n-i}(Z / K) \longrightarrow K \text {. }
$$

De plus, si $X$ est lisse, c'est un accouplement parfait.

- Si $X$ et $Y$ sont deux variétés sur $k$, on a des morphismes canoniques :

$$
\begin{gathered}
H_{\text {rig }}^{*}(X / K) \otimes H_{\text {rig }}^{*}(Y / K) \longrightarrow H_{\text {rig }}^{*}(X \times Y / K), \\
H_{c, \text { rig }}^{*}(X / K) \otimes H_{c, \text { rig }}^{*}(Y / K) \longrightarrow H_{c, \text { rig }}^{*}(X \times Y / K) .
\end{gathered}
$$

Le second est un isomorphisme. Si $X$ et $Y$ sont lisses, le premier est aussi un isomorphisme.

- La cohomologie rigide et la cohomologie rigide à support compact commutent à toute extension $K^{\prime} / K$ induite par un homomorphisme d'anneaux de valuation discrète.

REmARQue 1.2. - Grâce à la dernière partie du théorème, nous omettrons le corps $K$ dans la notation quand il n'y aura pas d'ambiguïté.

On a aussi :

Proposition 1.3 (Berthelot). — Avec les notations précédentes, si on se donne deux fermés $Z \subset Z^{\prime} \subset X$, on a une suite exacte longue d'excision

$$
\begin{aligned}
\cdots \rightarrow H_{Z-Z^{\prime}, \text { rig }}^{i-1}\left(X-Z^{\prime}\right) \longrightarrow & H_{Z^{\prime}, \text { rig }}^{i}(X) \longrightarrow H_{Z, \text { rig }}^{i}(X) \\
& \longrightarrow H_{Z-Z^{\prime}, \text { rig }}^{i}\left(X-Z^{\prime}\right) \longrightarrow H_{Z^{\prime}, \text { rig }}^{i+1}(X) \rightarrow \cdots .
\end{aligned}
$$

1.2. Rappels sur les cycles et les diviseurs. - Nous allons donner ici quelques définitions et propriétés relatives aux cycles et aux diviseurs. L'intégralité des résultats cités se trouve dans [13], [12] ou dans [EGAIV, 21]. Commençons par les pseudo-diviseurs [13].

DÉFInition 1.4. - Soit $X$ une variété. Un pseudo-diviseur sur $X$ est un triplet $(\mathscr{L}, Z, s)$ où $\mathscr{L}$ est un faisceau inversible sur $X, Z$ un fermé de $X$ et $s$ une trivialisation de la restriction de $\mathscr{L}$ à $X-Z$. Le fermé $Z$ s'appellera le support du pseudo-diviseur. Nous appellerons bon pseudo-diviseur un pseudo-diviseur dont le support est localement l'ensemble des zéros d'une section de $\mathscr{O}_{X}$.

Remarque 1.5. - Un pseudo-diviseur de support $X$ est un faisceau inversible.

REMARque 1.6. - Nous ne considérerons dans la suite, sauf mention explicite du contraire, que des bons pseudo-diviseurs (voir remarque 3.1).

TOME $131-2003-\mathrm{N}^{\mathrm{O}} 1$ 
Soit $D$ un diviseur de Cartier [EGAIV, 21]; on notera $\mathscr{O}(D)$ le faisceau associé et $|D|$ son support. On peut alors associer à tout diviseur de Cartier le pseudo-diviseur $\left(\mathscr{O}(D),|D|, s_{D}\right)$ où $s_{D}$ est la section canonique de $\mathscr{O}(D)$ (la restriction de $D$ à $X-|D|$ est effective).

Pour tout morphisme $f: X^{\prime} \rightarrow X$, un pseudo-diviseur $D=(\mathscr{L}, Z, s)$ admet une image inverse

$$
f^{*}(D)=\left(f^{*} \mathscr{L}, f^{-1}(Z), f^{*}(s)\right) .
$$

De plus si $D$ est un bon pseudo-diviseur, $f^{*}(D)$ est un bon pseudo-diviseur.

$\mathrm{Si}$ on se donne deux pseudo-diviseurs $D=(\mathscr{L}, Z, s)$ et $D^{\prime}=\left(\mathscr{L}^{\prime}, Z^{\prime}, s^{\prime}\right)$ sur $X$, on peut définir le pseudo-diviseur somme par

$$
D+D^{\prime}=\left(\mathscr{L} \otimes \mathscr{L}^{\prime}, Z \cup Z^{\prime}, s \otimes s^{\prime}\right) .
$$

Là encore si $D$ et $D^{\prime}$ sont deux bons pseudo-diviseurs, $D+D^{\prime}$ est un bon pseudo-diviseur.

Si on se fixe $Z$ un fermé de $X$, on notera $\operatorname{Div}_{Z}(X)$ l'ensemble des pseudodiviseurs de support $Z$ qui dans ce cas hérite naturellement d'une structure de groupe abélien.

Nous allons finir ces rappels avec l'homologie de Chow [12], [13]. On gradue le groupe des cycles par la dimension

$$
Z_{\bullet}(X)=\bigoplus_{k} Z_{k}(X)
$$

où $Z_{k}(X)$ est le groupe abélien libre engendré par les sous-schémas fermés intègres de dimension $k$. Un cycle $z \in Z \bullet(X)$ est dit rationnellement équivalent à zéro s'il existe un morphisme propre $\pi: Y \rightarrow X$ et un diviseur de Cartier principal $D$ sur $Y$ tel que

$$
z=\pi_{*}([D])
$$

Les cycles rationnellement équivalents à zéro forment un sous-groupe gradué de $Z_{\bullet}(X)$ et le groupe quotient est appelé homologie de Chow et noté $A \bullet(X)$.

Proposition 1.7. - Les morphismes de fonctorialité ci-dessus passent au quotient par l'équivalence rationnelle. Précisément, tout morphisme propre $f: X \rightarrow Y$ définit un morphisme $f_{*}: A_{\bullet}(X) \rightarrow A_{\bullet}(Y)$ et tout morphisme plat $f: X \rightarrow Y$ un morphisme $f^{*}: A_{\bullet}(Y) \rightarrow A_{\bullet}(X)$.

Si on suppose qu'il existe une immersion fermée de notre variété $X$ dans une variété lisse, on peut définir la cohomologie de Chow $A^{\bullet} X[13,8.3 .13]$. Pour cela on regarde les morphismes $f: X \rightarrow Y$ où $Y$ est une variété lisse. On note $(Y, f)$ une telle donnée. On considère $\mathscr{C}(X)$ la catégorie dont les objets sont les $(Y, f)$. Un morphisme de $(Y, f)$ dans $\left(Y^{\prime}, f^{\prime}\right)$ est la donnée d'une application $g: Y \rightarrow Y^{\prime}$ telle que $f^{\prime}=g \circ f$. On pose alors

$$
A^{\bullet} X=\underset{\mathscr{C}(X)}{\lim _{\longrightarrow}} A^{\bullet} Y
$$

BULLETIN DE LA SOCIÉtÉ MATHÉMATIQUE DE FRANCE 
où $A^{\bullet} Y$ est le groupe de Chow classique des cycles modulo l'équivalence rationnelle gradué par la codimension.

1.3. Intersections de cycles. - La théorie de l'intersection que nous utiliserons dans cette article est celle définie par Fulton dans $[13,8]$.

On se donne $Y$ une variété lisse de dimension $n, y$ dans $A_{\ell} Y, X$ une variété, $f: X \rightarrow Y$ et $x \in A_{k} X$. On construit alors [13,8.1] l'intersection de $x$ et $y$ :

$$
x \bullet_{f} y \in A_{m}(Z),
$$

où $Z=|x| \cap f^{-1}|y|$ et $m=k+\ell-n$. Cela permet de construire un accouplement entre l'homologie et la cohomologie de Chow

$$
A_{p} X \otimes A^{q} X \stackrel{\cap}{\longrightarrow} A_{p-q} X
$$

de la manière suivante : pour tout $x \in A_{p} X$ et $y \in A^{q} X$, on choisit $f: X \rightarrow Y$ avec $Y$ lisse et $\tilde{y} \in A^{q} Y$ pour représenter $y$. Alors on pose

$$
x \cap y=x \bullet_{f} \tilde{y} .
$$

\section{Homologie rigide - Formalisme de Bloch-Ogus}

Nous allons définir la notion d'homologie rigide. Cela nous permettra de construire la classe fondamentale d'une variété. On obtiendra ainsi un formalisme de théorie de dualité de Poincaré au sens de Bloch-Ogus [10].

On se fixe $\mathscr{V}$ un anneau de valuation discrète de corps résiduel $k$ et on note $K$ son corps des fractions.

On se donne une $k$-variété $X$; on pose

$$
H_{i}^{\mathrm{rig}}(X / K):=H_{c, \text { rig }}^{i}(X / K)^{\vee},
$$

où $H^{\vee}:=\operatorname{Hom}(H, K)$. On appelle ce groupe l'homologie rigide.

Supposons qu'il existe une $k$-variété $M$ lisse sur $k$ et une immersion fermée $X \hookrightarrow M$. En notant $N$ la dimension de $M$, on a grâce à la dualité de Poincaré ( $M$ est lisse) :

$$
H_{i}^{\text {rig }}(X / K) \stackrel{\sim}{\longrightarrow} H_{X, \text { rig }}^{2 N-i}(M / K) .
$$

Remarque 2.1. - Si $X$ est une variété lisse de dimension $n$, on a

$$
H_{i}^{\text {rig }}(X / K) \stackrel{\sim}{\longrightarrow} H_{\text {rig }}^{2 n-i}(X / K) .
$$

Regardons maintenant la variance de l'homologie rigide.

Proposition 2.2. - L'homologie rigide ainsi définie est contravariante par rapport aux immersions ouvertes et covariante vis-à-vis des morphismes propres.

TOME $131-2003-\mathrm{N}^{\mathrm{O}} 1$ 
Démonstration. - Soit $f: X \rightarrow Y$ un morphisme propre. On sait que la cohomologie à support compact est contravariante. Il existe donc pour tout $i$ :

$$
f^{*}: H_{c, \text { rig }}^{i}(Y / K) \longrightarrow H_{c, \text { rig }}^{i}(X / K) .
$$

En prenant la transposée on obtient le morphisme de fonctorialité voulu :

$$
f_{*}: H_{i}^{\mathrm{rig}}(X / K) \longrightarrow H_{i}^{\mathrm{rig}}(Y / K) .
$$

De plus, on sait aussi que la cohomologie rigide à support compact est covariante par rapport aux immersions ouvertes. Le même raisonnement nous permet de construire le morphisme de fonctorialité contravariante pour l'homologie rigide.

Nous allons énoncer quelques propriétés de l'homologie rigide qui découlent directement de celles de la cohomologie rigide à support compact.

Proposition 2.3. - On a :

- Soit $X$ une $k$-variété de dimension $n$; alors

$$
H_{i}^{\mathrm{rig}}(X / K)=0 \text { pour tout } i>2 n \text {. }
$$

- Si $X$ est une k-variété irréductible de dimension $n$, l'espace $H_{2 n}^{\mathrm{rig}}(X / K)$ est canoniquement isomorphe à $K$.

- Si $X$ est une $k$-variété et $Z \hookrightarrow X$ un sous-schéma fermé, on a la suite exacte longue :

$$
\begin{aligned}
\cdots \rightarrow H_{i+1}^{\mathrm{rig}}(X-Z / K) \longrightarrow & H_{i}^{\mathrm{rig}}(Z / K) \longrightarrow H_{i}^{\mathrm{rig}}(X / K) \\
& \longrightarrow H_{i}^{\mathrm{rig}}(X-Z / K) \longrightarrow H_{i-1}^{\mathrm{rig}}(Z / K) \rightarrow \cdots .
\end{aligned}
$$

- Soit $K^{\prime}$ une extension de $K$, d'anneau des entiers $\mathscr{V}^{\prime}$ et de corps résiduel $k^{\prime}$. On note $X^{\prime}=X \times_{k} k^{\prime}$ le schéma obtenu par changement de base. Il existe un isomorphisme canonique

$$
\varphi: H_{i}^{\mathrm{rig}}\left(X^{\prime} / K^{\prime}\right) \stackrel{\sim}{\longrightarrow} H_{i}^{\mathrm{rig}}(X / K) \otimes_{K} K^{\prime} .
$$

REMARQUE 2.4. - Grâce à la dernière propriété nous omettrons, comme pour la cohomologie, le corps $K$ quand il n'en résultera aucune ambiguïté.

En utilisant la première et la troisième assertion de la proposition on obtient :

Corollaire 2.5. - Si $X$ est une k-variété et si on note $X_{i}$ ses composantes irréductibles de dimension maximale $n$. On a

$$
H_{2 n}^{\mathrm{rig}}(X)=\bigoplus_{i} H_{2 n}^{\mathrm{rig}}\left(X_{i}\right)
$$


2.1. Classe fondamentale. - Soit $Z$ une $k$-variété intègre de dimension $d$, on va définir sa classe fondamentale qui est un élément de $H_{2 d}^{\text {rig }}(Z)$. Pour cela, on regarde le morphisme trace [6] :

$$
\operatorname{Tr}_{Z}: H_{c, \text { rig }}^{2 d}(Z) \longrightarrow K
$$

Il définit une classe $\eta_{Z} \in H_{2 d}^{\text {rig }}(Z)$.

Le morphisme trace est compatible à la restriction à un ouvert, à l'extension des scalaires et au morphisme de Künneth [6]. Ces faits se reformulent pour la classe fondamentale :

Proposition 2.6. - On a :

- La classe fondamentale est fonctorielle par rapport aux immersions ouvertes.

- Soit $K^{\prime}$ une extension de $K$, d'anneau des entiers $\mathscr{V}^{\prime}$ et de corps résiduel $k^{\prime}$. On note $X^{\prime}=X \times_{k} k^{\prime}$ le schéma obtenu par changement de base et

$$
\varphi: H_{2 n}^{\mathrm{rig}}\left(X^{\prime} / K^{\prime}\right) \longrightarrow H_{2 n}^{\mathrm{rig}}(X / K) \text {. }
$$

On $a \varphi\left(\eta_{X^{\prime}}\right)=\eta_{X}$.

- Soient $X$ et $Y$ deux $k$-variétés de dimension $n$ et $m$ respectivement. Le morphisme de Künneth

$$
H_{n+m}^{\mathrm{rig}}\left(X \times_{k} Y\right) \longrightarrow H_{n}^{\mathrm{rig}}(X) \otimes H_{m}^{\mathrm{rig}}(Y)
$$

envoie $\eta_{X \times Y}$ sur $\eta_{X} \otimes \eta_{Y}$

2.2. Cap-produit et formule de projection. - Nous allons définir le cap-produit et démontrer la formule de projection.

Pour toute immersion fermée $Y \hookrightarrow X$, nous définissons le cap-produit que nous noterons $\cap$ :

$$
\begin{aligned}
H_{i}^{\mathrm{rig}}(X) \otimes H_{Y, \text { rig }}^{j}(X) & \longrightarrow \quad H_{i-j}^{\mathrm{rig}}(Y), \\
(\varphi, x) & \longmapsto(y \mapsto \varphi(x \cup y))
\end{aligned}
$$

où on a identifié $H_{i}^{\mathrm{rig}}(X)\left(\operatorname{resp} . H_{i-j}^{\mathrm{rig}}(Y)\right)$ avec $H_{c, \text { rig }}^{i}(X)^{\vee}\left(\operatorname{resp} . H_{c, \text { rig }}^{i-j}(Y)^{\vee}\right)$ et noté $\cup$ le cup-produit $[6,2.2]$ :

$$
\cup: H_{Y, \text { rig }}^{j}(X) \otimes H_{c, \text { rig }}^{i-j}(Y) \longrightarrow H_{c, \text { rig }}^{i}(X) .
$$

Ce cup-produit est fonctoriel par rapport aux morphismes propres : pour tout diagramme cartésien

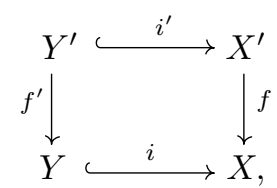

TOME $131-2003-\mathrm{N}^{\mathrm{O}} 1$ 
où $i$ et $i^{\prime}$ sont des immersions fermées et $f$ et $f^{\prime}$ des morphismes propres, et tous éléments $x \in H_{Y, \text { rig }}^{j}(X)$ et $y \in H_{c, \text { rig }}^{i-j}(Y)$, on a

$$
f^{*}(x \cup y)=f^{*}(x) \cup f^{*}(y) .
$$

On a une formule de projection qui se démontre formellement [30, IV.1.4.1] :

Proposition 2.7 (formule de projection). — Pour tout diagramme cartésien

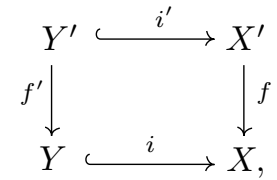

où $i$ et $i^{\prime}$ sont des immersions fermées et $f$ et $f^{\prime}$ des morphismes propres, et tous éléments $x^{\prime} \in H_{i}^{\mathrm{rig}}\left(X^{\prime}\right)$ et $x \in H_{Y, \mathrm{rig}}^{j}(X)$, on a

$$
f_{*}\left(x^{\prime}\right) \cap x=f_{*}^{\prime}\left(x^{\prime} \cap f^{*}(x)\right) .
$$

2.3. Le cas des variétés lisses. - Si on se donne $X$ une $k$-variété lisse de dimension $n$ et $Z$ un sous-schéma fermé intègre éventuellement singulier, il est plus habituel de regarder les groupes de cohomologie et non l'homologie. Les constructions précédentes se réécrivent alors de la manière suivante. On note $r=n-d$ la codimension de $Z$ dans $X$. La classe fondamentale de $Z$, encore notée $\eta_{Z}$, se construit donc comme un élément de $H_{Z \text {,rig }}^{2 r}(X)$. Si on note $\iota: Z \hookrightarrow X$ le morphisme d'immersion, on notera parfois cette classe $c(\iota)$. La dualité de Poincaré s'exprime alors avec ces notations sous la forme :

$$
c(\iota) \cap \eta_{X}=\eta_{Z}
$$

Si on suppose de plus que l'immersion fermée $Z \hookrightarrow X$ est la fibre spéciale d'une immersion fermée de $\mathscr{V}$-schémas quasi-projectifs $\mathscr{Z} \hookrightarrow \mathscr{X}$ on peut exprimer la classe fondamentale en terme de morphisme de Gysin (la construction du morphisme de Gysin en cohomologie rigide se trouve dans [7, 3.8]). En effet, le morphisme de Gysin est compatible à l'accouplement de dualité. On a donc :

Proposition 2.8. - Avec les notations précédentes, on note

$$
G_{Z / X}^{\mathrm{rig}}: H_{\text {rig }}^{0}(Z) \longrightarrow H_{Z, \text { rig }}^{2 r}(X)
$$

le morphisme de Gysin [6]. On a alors

$$
\eta_{Z}=G_{Z / X}^{\mathrm{rig}}(1)
$$

Pour finir, la formule de projection s'exprime alors :

BULLETIN DE LA SOCIÉTÉ MATHÉMATIQUe DE FRANCE 
Proposition 2.9 (formule de projection). - Pour tout diagramme cartésien

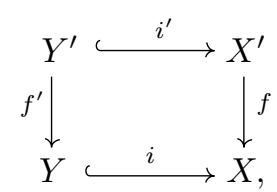

où $X$ et $X^{\prime}$ sont supposées lisses de dimensions respectives $n$ et $n^{\prime}$, $i$ et $i^{\prime}$ sont des immersions fermées et $f$ et $f^{\prime}$ des morphismes propres, et tous éléments $x^{\prime} \in H_{\text {rig }}^{i}\left(X^{\prime}\right)$ et $x \in H_{Y, \text { rig }}^{j}(X)$, on a

$$
f_{*}\left(x^{\prime}\right) \cup x=f_{*}^{\prime}\left(x^{\prime} \cup f^{*}(x)\right)
$$

où $f_{*}: H_{\mathrm{rig}}^{2 n^{\prime}-i}\left(X^{\prime}\right) \rightarrow H_{\mathrm{rig}}^{2 n-i}(X)$ est la transposée par les accouplements de Poincaré du morphisme $f^{*}: H_{c, \text { rig }}^{i}(X) \rightarrow H_{c, \text { rig }}^{i}\left(X^{\prime}\right) c^{\prime}$ 'est-à-dire que, via l'identification entre l'homologie et la cohomologie (remarque 2.1), c'est le morphisme de fonctorialité covariante en homologie.

2.4. Formalisme de Bloch-Ogus. - On peut regrouper les propriétés précédentes dans le théorème.

ThÉORÈme 2.10. - Soient $k$ un corps et $\mathscr{V}$ un anneau de Cohen pour $k$, $K$ le corps des fractions de $\mathscr{V}$. Le couple cohomologie rigide-homologie rigide forme une théorie de dualité de Poincaré au sens de Bloch-Ogus [10], [24]. Plus précisément, on a :

1) $H_{Y, \text { rig }}^{i}(X / K)$ est contravariant par rapport aux carrés cartésiens

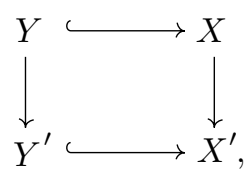

2) $H_{i}^{\mathrm{rig}}(X / K)$ est contravariant par rapport aux immersions ouvertes et covariant par rapport aux morphismes propres.

3) Pour $X$ une $k$-variété et $Z \subset Y \subset X$ des sous-schémas fermés, il existe une suite exacte longue

$$
\begin{aligned}
\cdots \rightarrow H_{Z, \text { rig }}^{i}(X / K) \rightarrow H_{Y, \text { rig }}^{i}(X / K) \rightarrow H_{Y-Z, \text { rig }}^{i}(X-Z / K) \rightarrow \\
H_{Z, \text { rig }}^{i+1}(X / K) \rightarrow \cdots,
\end{aligned}
$$

fonctorielle par rapport aux morphismes de fonctorialité de 1).

4) Pour tout $X, U$ un ouvert de $X$ et $Z$ un fermé de $X$ tel que $Z \subset U$, le morphisme $H_{Z \text {,rig }}^{i}(X / K) \rightarrow H_{Z, \text { rig }}^{i}(U / K)$ est un isomorphisme.

TOME $131-2003-\mathrm{N}^{\mathrm{O}} 1$ 
5) Si on a le diagramme cartésien suivant

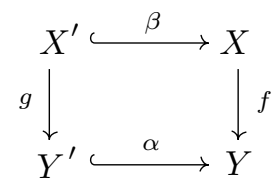

avec $\alpha$ et $\beta$ des immersions ouvertes et $f$ et $g$ des morphismes propres, le diagramme suivant est commutatif

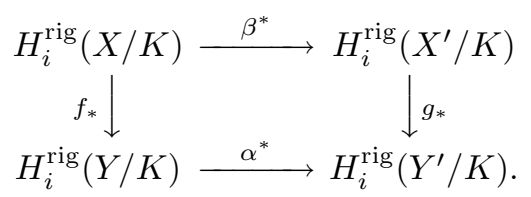

6) Si $i: Y \hookrightarrow X$ est une immersion fermée et si $\alpha: X-Y \hookrightarrow X$ est l'immersion ouverte complémentaire, il existe une suite exacte longue

$$
\begin{aligned}
\cdots \rightarrow H_{i+1}^{\mathrm{rig}}(X-Y / K) \longrightarrow & H_{i}^{\mathrm{rig}}(Y / K) \stackrel{i_{*}}{\longrightarrow} H_{i}^{\mathrm{rig}}(X / K) \\
& \stackrel{\alpha^{*}}{\longrightarrow} H_{i}^{\mathrm{rig}}(X-Y / K) \longrightarrow H_{i-1}^{\mathrm{rig}}(Y / K) \rightarrow \cdots
\end{aligned}
$$

qui est fonctorielle par rapport aux morphismes propres.

7) (cap-produit) Il existe un cap-produit pour tout $X$ et tout fermé $Y \hookrightarrow X$ :

$$
H_{i}^{\mathrm{rig}}(X / K) \otimes H_{Y, \text { rig }}^{j}(X / K) \stackrel{\cap}{\longrightarrow} H_{i-j}^{\mathrm{rig}}(Y / K) .
$$

8) (formule de projection) Pour tout diagramme cartésien

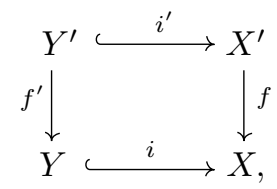

où $i$ et $i^{\prime}$ sont des immersions fermées et $f$ et $f^{\prime}$ des morphismes propres, et tous éléments $x^{\prime} \in H_{i}^{\mathrm{rig}}\left(X^{\prime} / K\right)$ et $x \in H_{Y, \mathrm{rig}}^{j}(X / K)$ on a :

$$
f_{*}\left(x^{\prime}\right) \cap x=f_{*}^{\prime}\left(x^{\prime} \cap f^{*}(x)\right) .
$$

9) (classe fondamentale) Pour toute variété irréductible $X$ de dimension $d$, il existe une classe fondamentale

$$
\eta_{X} \in H_{2 d}^{\mathrm{rig}}(X / K),
$$

qui est fonctorielle par rapport aux immersions ouvertes.

10) (dualité de Poincaré) Si $X$ est une $k$-variété irréductible et lisse de dimension $d$, et si $Y \hookrightarrow X$ est une immersion fermée, le morphisme

$$
H_{Y, \text { rig }}^{2 d-i}(X / K) \stackrel{\eta_{X} \cap}{\longrightarrow} H_{i}^{\text {rig }}(Y / K)
$$

BULLETIN DE LA SOCIÉTÉ MATHÉMATIQUE DE FRANCE 
est un isomorphisme.

11) La dualité de Poincaré est compatible aux suites exactes longues de 3).

REMARque 2.11. - Les axiomes habituels des théories de dualité de BlochOgus demandent une contravariance de l'homologie par rapport aux morphismes étales et pas seulement aux immersions ouvertes. De même, les fonctorialités des suites exactes sont requises dans le cas des morphismes étales. Cependant nous n'utiliserons dans la suite que la contravariance par rapport aux immersions ouvertes. Nous espérons y revenir ultérieurement.

\section{Classes de cohomologie associées à un pseudo-diviseur}

Nous allons montrer comment on peut associer canoniquement à un bon pseudo-diviseur $(\mathscr{L}, Z, s)$ sur $X$ une classe de cohomologie dans $H_{Z \text {,rig }}^{2}(X / K)$.

REMARque 3.1. - On supposera toujours, sauf mention explicite du contraire, que nos pseudo-diviseurs sont bons. L'hypothèse sera inutile si $X$ est lisse. En effet dans ce cas, le théorème de pureté nous dit que, si la codimension de $Z$ est supérieure strictement à 1 , on a $H_{Z \text {,rig }}^{2}(X)=0$.

Commençons par un peu de terminologie.

Soit $\mathscr{Y}=\operatorname{Spf}(\mathscr{A})$ un $\mathscr{V}$-schéma formel affine. Soit $X=\operatorname{Spec}(A)$ un fermé de la fibre spéciale $\mathscr{Y}_{0}$ de $\mathscr{Y}$ et $U$ un ouvert de $X$ défini par l'inversibilité d'une fonction $\bar{f} \in A$. On note $] X[$ (resp. $] U[$ ) le tube de $X$ (resp. $U$ ) dans la fibre générique rigide $Y=\mathscr{Y}_{K}$. Rappelons que dans cette situation on appelle voisinage strict de $] U[$ in $] X[$ (voir $[4,1.2]$ ) un ouvert admissible $V$ de $] X[$ tel que le recouvrement $(V] X-,U[)$ soit admissible. On notera alors $I$ l'idéal définissant $X$ dans $\mathscr{Y}$.

Remarque 3.2. - On considère aussi le cas $\bar{f}=1$ et $U=X$.

DÉFInItion 3.3. - Soient $V$ un voisinage strict de $] U$ [ dans $] X$ [ et $\rho$ un élément de $\Gamma\left(V, \mathscr{O}_{] X[}\right)$. On dit que $\rho$ se restreint en un élément inversible sur $U$ s'il existe $u \in \mathscr{A}$ et $v \in \mathscr{A}$ tels que $v$ soit inversible sur $V$, que $\rho=u / v$ (où on a noté encore $u$ et $v$ leurs images par le morphisme canonique $\mathscr{A} \rightarrow \mathscr{A} \otimes K$ ) et tels que leurs restrictions à $X$ notées $\bar{u} \in A$ et $\bar{v} \in A$ s'envoient dans des éléments inversibles de $A_{\bar{f}}$. De plus, si $\bar{u} \cdot \bar{v}^{-1}=1$ dans $A_{\bar{f}}$, on dira que $\rho$ se restreint à 1 sur $U$.

LEMme 3.4. - On reprend les notations précédentes. On a alors :

1) Si $\rho$ se restreint en un élément inversible sur $U$, alors il existe $V^{\prime} \subset V$, un voisinage strict de $] U[$ dans $] X\left[\right.$ tel que la restriction de $\rho \grave{a} V^{\prime}$ soit inversible.

TOME $131-2003-\mathrm{N}^{\mathrm{O}} 1$ 
2) Si, de plus, il se restreint à 1 sur $U$, on peut choisir $V^{\prime}$ tel que la série

$$
\log (\rho):=-\sum_{n=1}^{\infty} \frac{(1-\rho)^{n}}{n}
$$

converge et définisse donc un logarithme de $\rho$.

Démonstration. - 1) On peut supposer $v=1$ et $\rho=u$. Il existe $\bar{\alpha} \in A$ et $k \in \mathbb{N}$ tels que

$$
\bar{u} \cdot \bar{\alpha}=\bar{f}^{k} .
$$

En choisissant un relèvement $\alpha \in \mathscr{A}$ de $\bar{\alpha}$ et un relèvement $f \in \mathscr{A}$ de $\bar{f}$, on voit qu'il existe $c \in I$ tel que

$$
u \cdot \alpha=f^{k}+c .
$$

On utilise alors les voisinages standard. On considère deux suites $\left(\lambda_{n}\right)$ et $\left(\eta_{n}\right)$ tendant vers 1 et telles que pour tout $n$ on ait :

$$
\eta_{n}<\lambda_{n}^{k}<1
$$

On se donne $\left(g_{\ell}\right)_{\ell}$ un système de générateurs de l'idéal $I$. On pose alors :

$$
V_{n}=\{x \in] X\left[;|f(x)| \geqslant \lambda_{n}, \forall \ell,\left|g_{\ell}(x)\right| \leqslant \eta_{n}\right\} .
$$

On note $V=\bigcup_{n} V_{n}$. C'est un voisinage strict de $] U[$ dans $] X[$ (voir $[4,1.2 .4]$ ).

Pour tout $x$ dans cet ouvert on a par définition :

$$
\left|\frac{c}{f^{k}}(x)\right|<1
$$

Donc pour tout $x \in V$, la série

$$
\sum_{\ell=0}^{\infty}(-1)^{\ell}\left(\frac{c}{f^{k}}(x)\right)^{\ell}
$$

converge. Nous la noterons $\left(1+c / f^{k}\right)^{-1}$. La fonction définie sur $V$ par

$$
\rho^{-1}=\frac{\alpha}{f^{k}}\left(1+\frac{c}{f^{k}}\right)^{-1}
$$

est alors l'inverse de $\rho$.

2) Comme ci-dessus, il existe alors $\bar{\alpha} \in A$ et $k \in \mathbb{N}$ tel que

$$
\bar{\alpha} \cdot \bar{v}=\bar{f}^{k} \quad \text { et } \quad \bar{\alpha} \cdot \bar{u}=\bar{f}^{k} .
$$

En choisissant un relèvement $\alpha \in \mathscr{A}$ de $\bar{\alpha}$ et un relèvement $f \in \mathscr{A}$ de $\bar{f}$, on voit qu'il existe $c \in I$ et $d \in I$ tels que

$$
v \cdot \alpha=f^{k}+c \quad \text { et } \quad u \cdot \alpha=f^{k}+d .
$$

BULLETIN DE LA SOCIÉTÉ MATHÉMATIQUe DE FRANCE 
On sait alors ( $c f$. ci-dessus) qu'il existe un voisinage strict $V$ de $] U$ [ dans $] X[$ tel que les restrictions à $\mathrm{V}$ de $u, v, \alpha, f, f+c$ et $f+d$ soient inversibles. On a alors

$$
\rho=\frac{u}{v}=\left(1+\frac{d}{f^{k}}\right)\left(1+\frac{c}{f^{k}}\right)^{-1}=1+t,
$$

où $t$ est une fonction définie sur $V$ et vérifiant

$$
\forall x \in V, \quad|t(x)|<1 \text {. }
$$

Dès lors, la série

$$
\log (\rho):=-\sum_{n=1}^{\infty} \frac{(1-\rho)^{n}}{n}
$$

converge sur $V$.

On aura aussi besoin du lemme suivant qui se démontre formellement :

LEMme 3.5. - Soit $V$ un ouvert de $] X[$ et $(f, g)$ deux fonctions sur $V$ telles que pour tout $x \in V$ on ait $|f(x)|<1$ et $|g(x)|<1$; alors on a

$\log ((1+f)(1+g))=\log (1+f)+\log (1+g) \quad$ et $\quad \mathrm{d} \log (1+f)=\mathrm{d} f /(1+f)$.

On va maintenant construire la classe de cohomologie associée à un pseudodiviseur sur $X$.

3.1. Cas des variétés propres. - Dans toute cette partie, on se fixe $X$ une variété propre et $\mathscr{V}$ un anneau de valuation discrète de corps résiduel $k$. On se donne de plus une immersion fermée $X \hookrightarrow \mathscr{Y}$ où $\mathscr{Y}$ est un schéma formel $\operatorname{sur} \operatorname{Spf}(\mathscr{V})$ lisse au voisinage de $X$.

Soit $D=(\mathscr{L}, Z, s)$ un bon pseudo-diviseur. Nous allons utiliser une construction à base de cocycle de Čech.

LEMME 3.6. - Il existe un recouvrement affine $\mathfrak{U}=\left(\mathscr{U}_{i}\right)_{i \in \Lambda}$ de $\mathscr{Y}$ tel que si on note $\mathfrak{U}_{X}$ le recouvrement induit par $\mathfrak{U}$ sur $X$, le faisceau $\mathscr{L}$ soit trivialisé sur $\mathfrak{U}_{X}$.

Démonstration. - Quitte à prendre un recouvrement affine de $\mathscr{Y}$, on peut supposer que $\mathscr{Y}$ et $X$ sont affines. On pose alors $\mathscr{Y}=\operatorname{Spf}(\mathscr{A})$ et $X=\operatorname{Spec}(A)$ où $A=\mathscr{A} / I$. On choisit un recouvrement $\left(X_{i}\right)_{i \in \Lambda}$ de $X$ trivialisant $\mathscr{L}$. Quitte à raffiner le recouvrement, on peut supposer que pour tout $i \in \Lambda$, il existe $f_{i} \in A$ tel que $X_{i}=D\left(f_{i}\right)$. Pour tout $i \in \Lambda$, on choisit alors $\tilde{f}_{i}$ un relèvement de $f_{i}$ dans $\mathscr{A}$, les ouverts $\operatorname{Spf}\left(\widehat{\mathscr{A}_{\tilde{f}_{i}}}\right)$ recouvrent alors l'image de $X$ dans $\mathscr{Y}$. En rajoutant des ouverts de $\mathscr{Y}-X$, on obtient le recouvrement voulu.

On choisit donc un tel recouvrement $\mathfrak{U}=\left(\mathscr{U}_{i}\right)_{i \in \Lambda}$. Dès lors on considère $\mathfrak{U}_{X}=\left(X_{i}\right)_{i \in \Lambda}$ le recouvrement induit sur $X$ et on notera $\mathfrak{U}_{K}$ le recouvrement induit sur la fibre générique rigide. Pour tout $i \in I$, on notera $\mathscr{U}_{i}=\operatorname{Spf}\left(\mathscr{A}_{i}\right)$, $X_{i}=\operatorname{Spec}\left(A_{i}\right)$ où $A_{i}=\mathscr{A}_{i} / I_{i}$. On peut de plus supposer, car $D$ est un bon

TOME $131-2003-\mathrm{N}^{\mathrm{O}} 1$ 
pseudo-diviseur, que pour tout $i, Z_{i}=Z \cap X_{i}$ soit définit par une équation $h_{i} \in A_{i}$ (si $Z_{i}=\varnothing$ on prend $h_{i}=1$ ). On notera $U=X-Z$ et $U_{i}=X_{i}-Z_{i}$ ainsi que $j: U \hookrightarrow X$ le morphisme d'inclusion.

REMARQUe 3.7. - On notera avec un multi-indice $\underline{i}=\left(i_{0}, \ldots, i_{n}\right)$ les mêmes objets définis par rapport à l'ouvert $\mathscr{U}_{\underline{i}}=\mathscr{U}_{i_{0}} \cap \cdots \cap \mathscr{U}_{i_{n}}$. En particulier, on écrira

$$
h_{\underline{i}}:=h_{i_{0}} \cdots h_{i_{n}} .
$$

On se donne une trivialisation $\varphi_{i}: \mathscr{O}_{X_{i}} \stackrel{\sim}{\rightarrow} \mathscr{L}_{\mid X_{i}}$ de $\mathscr{L}$. On pose

$$
\phi_{i}=\varphi_{i}(1) \in \mathscr{L}_{\mid X_{i}} .
$$

À cette trivialisation on associe un cocycle $(u) \in Z^{1}\left(\mathfrak{U}_{X}, \mathscr{O}_{X}^{*}\right)$ de la manière classique, en posant

$$
\phi_{j}=u_{i j} \phi_{i}
$$

Pour tout $i$, on note $s_{i} \in \Gamma\left(U_{i}, \mathscr{L}\right)$ la restriction à $U_{i}$ de la section $s$ de $\mathscr{L}$. Il existe alors pour tout $i$, une unique section $a_{i} \in \Gamma\left(U_{i}, \mathscr{O}_{X}^{*}\right)$ telle que

$$
\phi_{i}=a_{i} s_{i} .
$$

On a dans $\left(A_{i j}\right)_{h_{i j}}$ :

$$
u_{i j}=\frac{a_{j}}{a_{i}}
$$

Ci-dessus, on a noté encore $u_{i j}$ l'image de $u_{i j}$ par la flèche de localisation $A_{i j} \rightarrow\left(A_{i j}\right)_{h_{i j}}$.

À cette donnée on va associer une classe dans $H^{2}\left(\mathfrak{U}_{K},\left(\Omega_{] X[}^{\star} \rightarrow j_{U}^{\dagger} \Omega_{] X[}^{\star}\right)_{s}\right)$. Pour cela on va construire un élément dans

$$
\begin{aligned}
& C^{2}\left(\mathfrak{U}_{K},\left(\Omega_{] X[}^{\star} \rightarrow j_{U}^{\dagger} \Omega_{] X[}^{\star}\right)_{s}\right) \\
& \quad=C^{2}\left(\mathfrak{U}_{K}, \mathscr{O}_{] X[}\right) \oplus C^{1}\left(\mathfrak{U}_{K}, \Omega_{] X[}^{1} \oplus j_{U}^{\dagger} \mathscr{O}_{] X[}\right) \oplus C^{0}\left(\mathfrak{U}_{K}, \Omega_{] X[}^{2} \oplus j_{U}^{\dagger} \Omega_{] X[}^{1}\right) .
\end{aligned}
$$

On va regarder la somme ci-dessus terme à terme :

- $C^{1}\left(\mathfrak{U}_{K}, \Omega_{] X[}^{1}\right)$ : on choisit des relèvements $\widetilde{u}_{i j} \in \mathscr{A}_{i j}$ de $u_{i j}$. Vue comme fonction analytique rigide sur $] X_{i j}\left[, \widetilde{u}_{i j}\right.$ se restreint alors en un élément inversible sur $X$. Le lemme 3.4 nous dit alors qu'elle est inversible comme fonction analytique sur $] X_{i j}[$. On pose alors

$$
\mu_{i j}=\frac{\mathrm{d} \widetilde{u}_{i j}}{\widetilde{u}_{i j}}
$$

On a donc $(\mu) \in C^{1}\left(\mathfrak{U}_{K}, \Omega_{] X[}^{1}\right)$.

- $C^{2}\left(\mathfrak{U}_{K}, \mathscr{O}_{] X[}\right)$ : on est parti d'un cocycle $u_{i j}$. Cependant, le choix des relèvements fait apparaître un défaut de cocycle dans le terme $\mu$ ci-dessus. Il y a donc lieu d'apporter un terme correctif.

BULLETIN DE LA SOCIÉtÉ MATHÉMATIQUE DE FRANCE 
Pour $i, j, k$ trois indices, le terme $\widetilde{u}_{i j} \cdot \widetilde{u}_{i k}^{-1} \cdot \widetilde{u}_{j k}$ se restreint à 1 sur $X$. En effet $(u)$ est, par définition, un cocycle. On peut donc prendre son logarithme par le lemme 3.4. On pose

$$
\nu_{i j k}:=-\log \left(\widetilde{u}_{i j} \cdot \widetilde{u}_{i k}^{-1} \cdot \widetilde{u}_{j k}\right) \in \Gamma(] X_{i j k}\left[, \mathscr{O}_{] X}\right) .
$$

Les éléments $\mu_{i j}$ et $\nu_{i j k}$ forment un 2-cocycle de Čech du complexe $\Omega_{] X[}^{\star}$ (voir la démonstration de la proposition 3.9).

Regardons maintenant les termes surconvergents :

- $C^{0}\left(\mathfrak{U}_{K}, j_{U}^{\dagger} \Omega_{] X[}^{1}\right)$ : il existe $r \in \mathbb{N}$ et $t_{i} \in A_{i}$ tels que pour tout $i$ on ait

$$
h_{i}^{r} a_{i}=t_{i} .
$$

On choisit alors des relèvements $\widetilde{h}_{i}$ et $\widetilde{t}_{i}$ de $h_{i}$ et $t_{i}$ dans $\mathscr{A}_{i}$. Par définition, les fonctions analytiques $\widetilde{h}_{i}$ et $\widetilde{t}_{i}$ se restreignent en des éléments inversibles sur $U_{i}$. Il existe donc un voisinage strict $V_{i}$ de $] U_{i}$ [ dans $] X_{i}$ [ tel que la restriction de $\widetilde{h}_{i}$ et $\widetilde{t}_{i}$ à $V_{i}$ soient inversibles (lemme 3.4). Les différentielles

$$
\sigma_{i}=\frac{\mathrm{d} \widetilde{t_{i}}}{\widetilde{t_{i}}}-r \frac{\mathrm{d} \widetilde{h_{i}}}{\widetilde{h_{i}}} \in \Gamma\left(V_{i}, \Omega_{] X[}^{1}\right)
$$

définissent un élément de $C^{0}\left(\mathfrak{U}_{K}, j_{U}^{\dagger} \Omega_{] X[}^{1}\right)$.

- $C^{1}\left(\mathfrak{U}_{K}, j_{U}^{\dagger} \mathscr{O}_{X[}\right)$ : il faut ajouter un terme correctif afin de construire un élément de $Z^{2}\left(\mathfrak{U}_{K},\left(\Omega_{] X[}^{\star} \rightarrow j_{U}^{\dagger} \Omega_{] X[}^{\star}\right)_{s}\right)$.

D'après ce qui précède, on sait qu'il existe un voisinage strict $V_{i j}$ de $] U_{i j}[$ dans $] X_{i j}\left[\right.$ tel que les restrictions à $V_{i j}$ de $\widetilde{u}_{i j}, \widetilde{h}_{i}, \widetilde{h}_{j}, \widetilde{t}_{i}$ et $\widetilde{t}_{j}$ sont inversibles. On regarde alors

$$
\omega_{i j}=\widetilde{u}_{i j} \frac{\widetilde{t}_{i}{\widetilde{h_{j}}}^{r}}{\widetilde{t}_{j}{\widetilde{h_{i}}}^{r}}
$$

D'après l'équation (2), la section $\omega_{i j}$ se restreint à 1 sur $U_{i j}$. On sait alors par le lemme 3.4 que, quitte à restreindre le voisinage strict $V_{i j}$, on peut prendre le logarithme de $\omega_{i j}$. On pose

$$
\tau_{i j}:=\log \left(\omega_{i j}\right) .
$$

Les éléments $\mu_{i j}, \nu_{i j k}, \sigma_{i}, \tau_{i j}$ et 0 pour la composante dans $C^{0}\left(\mathfrak{U}_{K}, \Omega_{] X[}^{2}\right)$, définissent dans $C^{2}\left(\mathfrak{U}_{K},\left(\Omega_{] X[}^{\star} \rightarrow j_{U}^{\dagger} \Omega_{] X[}^{\star}\right)_{s}\right)$ un élément noté $\zeta$.

Remarque 3.8. - Si $Z=X$ et donc $U$ est vide, les faisceaux $j_{U}^{\dagger} \Omega_{] X[}^{i}$ sont nuls. L'élément $\zeta$ appartient à $C^{2}\left(\mathfrak{U}_{K},\left(\Omega_{] X[}^{\star}\right)\right.$ et est défini par $\mu_{i j}$ et $\nu_{i j k}$.

Proposition 3.9. - Avec les notations précédentes, on a

$$
\Delta(\zeta)=0
$$

où $\Delta$ est la différentielle totale du complexe $C^{\star}\left(\mathfrak{U}_{K},\left(\Omega_{] X[}^{\star} \rightarrow j_{U}^{\dagger} \Omega_{] X[}^{\star}\right)_{s}\right)$.

TOME $131-2003-\mathrm{N}^{\mathrm{O}} 1$ 
Démonstration. - Avant de commencer, rappelons les conventions de signes. Si on note $(L, \mathrm{~d})$ le complexe $\left(\Omega_{] X[}^{\star} \rightarrow j_{U}^{\dagger} \Omega_{] X[}^{\star}\right)_{s}$ (la définition de d est donnée dans la matrice (1) dans la partie 1) et $\delta$ la différentielle de Čech, la différentielle totale $\Delta$ est la somme des $\Delta_{p, q}$ où

$$
\begin{aligned}
& \Delta_{p, q}: C^{p}\left(\mathfrak{U}_{K}, L^{q}\right) \longrightarrow C^{p+1}\left(\mathfrak{U}_{K}, L^{q}\right) \oplus C^{p}\left(\mathfrak{U}_{K}, L^{q+1}\right), \\
& x \quad \longmapsto \quad \delta(x)+(-1)^{p} \mathrm{~d}(x) .
\end{aligned}
$$

Avec ces notations, pour montrer que $\zeta$ est un cocycle, il suffit de montrer que $\nu_{i j k}$ et $\mu_{i j}$ définissent un cocycle de $C^{\star}\left(\mathfrak{U}_{K}, \Omega_{1 X[}^{\star}\right)$ et que l'on a

$$
u\left(\nu_{i j k}\right)+\delta\left(\tau_{i j}\right)=0, \quad \delta\left(\sigma_{i}\right)+\mathrm{d}\left(\tau_{i j}\right)=u\left(\mu_{i j}\right) \quad \text { et } \quad \mathrm{d}\left(\sigma_{i}\right)=0 .
$$

- Pour montrer que $\nu_{i j k}$ et $\mu_{i j}$ définissent un cocycle de $C^{\star}\left(\mathfrak{U}_{K}, \Omega_{] X[}^{\star}\right)$, il faut montrer que

$$
\mathrm{d}\left(\nu_{i j k}\right)+\delta\left(\mu_{i j}\right)=0
$$

Or on a

$$
\delta\left(\mu_{i j}\right)=\frac{\mathrm{d} \widetilde{u}_{i j}}{\widetilde{u}_{i j}}-\frac{\mathrm{d} \widetilde{u}_{i k}}{\widetilde{u}_{i k}}+\frac{\mathrm{d} \widetilde{u}_{j k}}{\widetilde{u}_{j k}}=\frac{\mathrm{d}\left(\widetilde{u}_{i j} \cdot \widetilde{u}_{i k}^{-1} \cdot \widetilde{u}_{j k}\right)}{\left(\widetilde{u}_{i j} \cdot \widetilde{u}_{i k}^{-1} \cdot \widetilde{u}_{j k}\right)} .
$$

On conclut en utilisant le lemme 3.5.

- Montrons que $u\left(\nu_{i j k}\right)+\delta\left(\tau_{i j}\right)=0$. On a :

$\delta\left(\tau_{i j}\right)=\log \left(\omega_{i j}\right)-\log \left(\omega_{i k}\right)+\log \left(\omega_{j k}\right)=\log \left(\omega_{i j} \cdot \omega_{i k}^{-1} \cdot \omega_{j k}\right)=-u\left(\nu_{i j k}\right)$.

- Montrons que $\delta\left(\sigma_{i}\right)+d\left(\tau_{i j}\right)=u\left(\mu_{i j}\right)$. On a :

$$
\begin{aligned}
\delta\left(\sigma_{i}\right)+d\left(\tau_{i j}\right) & =\frac{\mathrm{d} \widetilde{t}_{j}}{\widetilde{t_{j}}}-\frac{\mathrm{d} \widetilde{t}_{i}}{\widetilde{t}_{i}}-r \frac{\mathrm{d} \widetilde{h_{j}}}{\widetilde{h_{j}}}+r \frac{\mathrm{d} \widetilde{h_{i}}}{\widetilde{h_{i}}}+\mathrm{d} \log \left(\omega_{i j}\right) \\
& =\frac{\mathrm{d}\left(\omega_{i j} \cdot\left(\widetilde{t_{j}}{\widetilde{h_{i}}}^{r}\right) /\left(\widetilde{t_{i}}\right){\widetilde{h_{j}}}^{r}\right)}{\left(\omega_{i j} \cdot\left(\widetilde{t}_{j}{\widetilde{h_{i}}}^{r}\right) /\left(\widetilde{t_{i}}\right){\widetilde{h_{j}}}^{r}\right)}=u\left(\frac{\mathrm{d} \widetilde{u}_{i j}}{\widetilde{u_{i j}}}\right) .
\end{aligned}
$$

Il est immédiat que $\mathrm{d}\left(\sigma_{i}\right)=0$.

On a donc associé un élément dans $Z^{2}\left(\mathfrak{U}_{K},\left(\Omega_{] X[}^{\star} \rightarrow j_{U}^{\dagger} \Omega_{] X[}^{\star}\right)_{s}\right)$ à notre pseudo-diviseur. Cependant cet élément dépend des choix que nous avons fait lors de la construction : choix du cocycle, choix des relèvements. Nous allons montrer que, par contre, la classe de cohomologie dans le $H^{2}\left(\mathfrak{U}_{K},\left(\Omega_{] X[}^{\star} \rightarrow j_{U}^{\dagger} \Omega_{] X[}^{\star}\right)_{s}\right)$ n'en dépend pas.

On reprend les notations précédentes et on se donne une deuxième trivialisation de $\mathscr{L}$ :

$$
\varphi_{i}^{\prime}: \mathscr{O}_{X_{i}} \longrightarrow \mathscr{L}_{X_{i}}
$$

On lui associe un second cocycle $\left(u^{\prime}\right) \in Z^{1}\left(\mathfrak{U}_{X}, \mathscr{O}_{X}^{*}\right)$ représentant $\mathscr{L}$, et on choisit comme ci-dessus des relèvements $\widetilde{u}_{i j}$ (resp. $\widetilde{u}_{i j}^{\prime}$ ) de $u_{i j}$ (resp. $u_{i j}^{\prime}$ ).

BULlETiN DE LA SOCIÉtÉ MATHÉMATIQUE DE FRANCE 
Proposition 3.10. - En notant $c_{1}$ (resp. $\left.c_{1}^{\prime}\right)$ l'élément de $Z^{2}\left(\mathfrak{U}_{K},\left(\Omega_{] X[}^{\star} \rightarrow\right.\right.$ $\left.j_{U}^{\dagger} \Omega_{]_{X}[}^{\star}\right)_{s}$ ) obtenu comme ci-dessus avec les relèvements $\widetilde{u}_{i j}$ (resp. $\widetilde{u}_{i j}^{\prime}$ ), il existe $(\varepsilon) \in C^{1}\left(\mathfrak{U}_{K},\left(\Omega_{X X}^{\star} \rightarrow j_{U}^{\dagger} \Omega_{] X[}^{\star}\right)_{s}\right)$ tel que

$$
c_{1}^{\prime}-c_{1}=\Delta(\varepsilon) .
$$

Démonstration. - On veut définir $\varepsilon \in C^{1}\left(\mathfrak{U}_{K},\left(\Omega_{]_{X}}^{\star} \rightarrow j_{U}^{\dagger} \Omega_{]_{X}[}^{\star}\right)_{s}\right)$. On va comme ci-dessus le définir composante par composante. Posons $\phi_{i}^{\prime}=\varphi_{i}^{\prime}(1)$. Il existe $\theta \in C^{0}\left(\mathfrak{U}_{X}, \mathscr{O}_{X}^{*}\right)$ tel que $\phi_{i}^{\prime}=\theta_{i} \phi_{i}$ pour tout $i$, ce qui entraîne que

$$
u^{\prime}=u \delta(\theta) \text {. }
$$

Soit $a_{i} \in \Gamma\left(U_{i}, \mathscr{O}_{X}^{*}\right)$ (resp. $a_{i}^{\prime}$ ) l'unique section telle que $\phi_{i}=a_{i} s_{i}$ (resp. $\left.\phi_{i}^{\prime}=a_{i}^{\prime} s_{i}\right)$. On obtient alors $a_{i}^{\prime}=a_{i} \theta_{i}$ dans $\left(A_{i}\right)_{h_{i}}$ et on peut trouver $r \in \mathbb{N}$, $t_{i}, t_{i}^{\prime} \in A_{i}$ tels que

$$
h_{i}^{r} a_{i}=t_{i}, \quad h_{i}^{r} a_{i}^{\prime}=t_{i}^{\prime} .
$$

Il s'ensuit que $t_{i}^{\prime}=t_{i} \theta_{i}$ dans $\left(A_{i}\right)_{h_{i}}$.

On choisit des relèvements $\tilde{\theta}_{i}, \tilde{t}_{i}$ et $\tilde{t}_{i}^{\prime}$ de $\theta_{i}, t_{i}$ et $t_{i}^{\prime}$ dans $\mathscr{A}_{i}$. D'après le lemme 3.4, les $\tilde{\theta}_{i}$ sont inversibles comme fonctions analytiques sur $] X_{i}[$, et il existe un voisinage strict de $] U_{i}$ [ sur lequel les $\tilde{t}_{i}$ et les $\tilde{t}_{i}^{\prime}$ sont inversibles. On pose

$$
\kappa_{i j}:=\frac{\tilde{u}_{i j}^{\prime}}{\tilde{u}_{i j}} \cdot \frac{\tilde{\theta}_{i}}{\tilde{\theta}_{j}}, \quad \kappa_{i}:=\frac{\tilde{t}_{i} \tilde{\theta}_{i}}{\tilde{t}_{i}^{\prime}} .
$$

Les fonctions $\kappa_{i j}\left(\right.$ resp. $\left.\kappa_{i}\right)$ se restreignent à 1 sur $X_{i}$ (resp. $U_{i}$ ), ce qui permet de prendre leur logarithme. On peut donc définir $\varepsilon$ par

$$
\begin{aligned}
& \varepsilon=-\log \kappa_{i j}+\frac{\mathrm{d} \widetilde{\theta}_{i}}{\widetilde{\theta}_{i}}+\log \kappa_{i} \\
& \in C^{1}\left(\mathfrak{U}_{K}, \mathscr{O}_{] X[}\right) \oplus C^{0}\left(\mathfrak{U}_{K}, \Omega_{] X[}^{1}\right) \oplus C^{0}\left(\mathfrak{U}_{K}, j_{U}^{\dagger} \mathscr{O}_{] X[}\right) .
\end{aligned}
$$

Un calcul similaire à ceux qui précèdent montre alors notre égalité.

La proposition montre que la classe de cohomologie dans $\mathbb{H}^{2}\left(\mathfrak{U}_{K},\left(\Omega_{] X[}^{\star} \rightarrow\right.\right.$ $\left.\left.j_{U}^{\dagger} \Omega_{j X[}^{\star}\right)_{s}\right)$ ne dépend ni du choix de la trivialisation de $\mathscr{L}$, ni du choix du relèvement du cocycle $(u)$, ni du choix des relèvements des $t_{i}$ (pour ces deux derniers cas, il suffit d'appliquer le lemme en utilisant deux fois la même trivialisation). Il nous reste à montrer qu'elle ne dépend pas non plus ni du choix des sections $h_{i}$, ni du choix de leurs relèvements.

Supposons que l'on se donne, pour tout $i$, deux équations $h_{i}$ et $h_{i}^{\prime}$ de $Z$. On se fixe des relèvements de $h_{i}$ et $h_{i}^{\prime}$ dans $\mathscr{A}_{i}$ que nous noterons $\widetilde{h}_{i}$ et $\widetilde{h}_{i}^{\prime}$. On se fixe de plus les sections $a_{i}$, le cocycle $(u)$ ainsi qu'un relèvement de ce dernier. Dès lors, il existe $r \in \mathbb{N}$ et $t_{i}$ et $t_{i}^{\prime}$ des éléments de $A_{i}$ tels que

$$
h_{i}^{r} a_{i}=t_{i} \quad \text { et } \quad h_{i}^{\prime r} a_{i}=t_{i}^{\prime} .
$$

TOME $131-2003-\mathrm{N}^{\mathrm{O}} 1$ 
On considère alors la section $h_{i}^{\prime \prime}=h_{i} h_{i}^{\prime}$ et son relèvement $\widetilde{h_{i}^{\prime \prime}}=\widetilde{h}_{i}{\widetilde{h_{i}^{\prime}}}_{\text {. La section }}$ $h_{i}^{\prime \prime}$ est aussi une équation de $Z$ (on ne s'intéresse qu'à la structure réduite de $Z$ ). Dès lors, on considère la section $t_{i}^{\prime \prime}=t_{i}{h_{i}^{\prime r}}^{r}$ que l'on relève en $\widetilde{t_{i}^{\prime \prime}}=\widetilde{t_{i}} \cdot{\widetilde{h_{i}^{\prime}}}^{r}$. Il est alors immédiat que

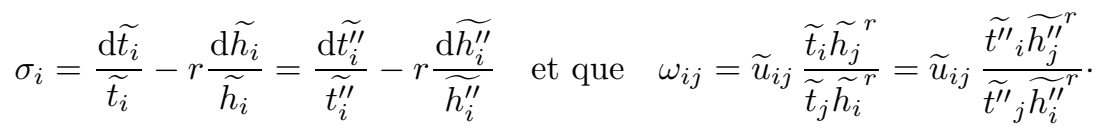

On en déduit par symétrie et en utilisant que la classe de cohomologie construite ne dépend pas du choix des relèvements des sections $t_{i}$, que la classe de cohomologie ne dépend pas ni du choix des sections $h_{i}$, ni des leurs relèvements.

Remarque 3.11. - On a vu (remarque 3.8) que si $Z=X$ l'élément $\zeta$ appartient à $C^{2}\left(\mathfrak{U}_{K}, \Omega_{] X[}^{\star}\right)$ et est défini par $\mu_{i j}$ et $\nu_{i j k}$. Si de plus le faisceau $\mathscr{L}$ se relève sur $\mathscr{Y}$ en un faisceau $\mathscr{L}_{\mathscr{Y}}$. On peut alors choisir comme cocyle $(u)$ représentant $\mathscr{L}$ la restriction d'un cocyle $(w)$ représentant $\mathscr{L}_{\mathscr{Y}}$. Dès lors en choisissant $w_{i j}$ comme relèvement de $u_{i j}$ dans le calcul précédent, on voit que l'élément $\zeta$ est défini seulement par $\mu_{i j}=\mathrm{d} w_{i j} / w_{i j}$.

Il est clair, de plus, que notre construction est compatible au raffinement du recouvrement $\mathfrak{U}$. On a donc associé à tout bon pseudo-diviseur $(\mathscr{L}, Z, s)$ une classe $c_{1}(\mathscr{L}, Z, s)$ dans $\check{H}^{2}(] X\left[,\left(\Omega_{] X[}^{\star} \rightarrow j_{U}^{\dagger} \Omega_{] X[}^{\star}\right)_{s}\right)$.

LEMME 3.12. - Avec les notations précédentes, l'image de $c_{1}(\mathscr{L}, Z, s)$ dans $H_{Z, \text { rig }}^{2}(X / K)$ ne dépend pas du plongement choisi.

Démonstration. - On se donne deux plongements $X \hookrightarrow \mathscr{Y}^{1}$ et $X \hookrightarrow \mathscr{Y}^{2}$ de $X$ dans des $\mathscr{V}$-schémas formels lisses au voisinage de $X$. En regardant le plongement diagonal $X \hookrightarrow \mathscr{Y}^{1} \times \mathscr{Y}^{2}$, on peut supposer qu'il existe un morphisme $p: \mathscr{Y}^{2} \rightarrow \mathscr{Y}^{1}$ faisant commuter le diagramme

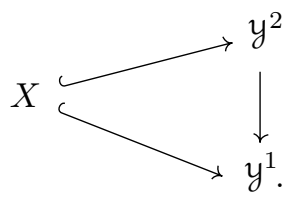

On se donne donc un recouvrement affine $\mathfrak{U}^{1}$ de $\mathscr{Y}^{1}$ dont la restriction à $X$ trivialise $\mathscr{L}$ et un recouvrement affine $\mathfrak{U}^{2}$ de $\mathscr{Y}^{2}$ qui soit compatible à $\mathfrak{U}^{1}$. C'est-à-dire que, pour tout ouvert $\mathscr{Y}_{i}^{1}$ du recouvrement $\mathfrak{U}^{1}$, on se donne un recouvrement de $\mathscr{Y}_{i}{ }^{2}=p^{-1} \mathscr{Y}_{i}{ }^{1}$. Tout ouvert $\mathscr{Y}_{i, j}^{2}$ de $\mathfrak{U}^{2}$, nous donne, en passant aux algèbres des carrés commutatifs

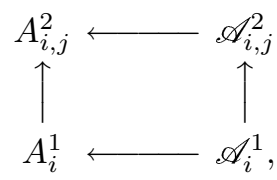

BULlETIN DE LA SOCIÉTÉ MATHÉMATIQUE DE FRANCE 
où $A_{i}^{1}$ (resp. $A_{i, j}^{2}$ ) sont les algèbres de $X \cap \mathscr{Y}_{i}^{1}$ (resp. $X \cap \mathscr{Y}_{i, j}^{2}$ ). Dès lors, on voit que l'on peut choisir, dans nos constructions, des relèvements qui soient compatibles au morphisme $p$.

DÉfinition 3.13. - On notera encore $c_{1}(\mathscr{L}, Z, s)$ l'image de cette classe dans $H_{Z \text {,rig }}^{2}(X / K)$. C'est la classe du pseudo-diviseur. Pour tout $Z$, on notera

$$
c_{1}: \operatorname{Div}_{Z}(X) \longrightarrow H_{Z, \text { rig }}^{2}(X / K)
$$

le morphisme ainsi défini.

En utilisant des diagrammes commutatifs similaires à ceux de la démonstration du lemme 3.12, on peut démontrer que nos classes sont fonctorielles :

Proposition 3.14 (fonctorialité). - Soient $f: X^{\prime} \rightarrow X$ un morphisme de variétés propres et $D$ un bon pseudo-diviseur $D=(\mathscr{L}, Z, s)$. Dans $H_{f^{-1}(Z), \text { rig }}^{2}(X)$, on a l'égalité

$$
f^{*} c_{1}(D)=c_{1}\left(f^{*} D\right)
$$

De plus, comme toutes nos constructions commutent au changement de corps de base, on a la proposition :

Proposition 3.15 (extension des scalaires). - Soit $K^{\prime}$ une extension non nécessairement finie de $K$, d'anneau des entiers $\mathscr{V}^{\prime}$ et de corps résiduel $k$. Pour tout bon pseudo-diviseur $D=(\mathscr{L}, Z, s)$ sur $X$, on note $D^{\prime}=\left(\mathscr{L}^{\prime}, Z^{\prime}, s^{\prime}\right)$ le pseudo-diviseur sur $X^{\prime}=X \times_{k} k^{\prime}$ obtenu par changement de base. Alors $D^{\prime}$ est un bon pseudo-diviseur et le morphisme

$$
H_{Z, \text { rig }}^{2}(X / K) \longrightarrow H_{Z^{\prime}, \text { rig }}^{2}\left(X^{\prime} / K^{\prime}\right)
$$

envoie $c_{1}(D)$ sur $c_{1}\left(D^{\prime}\right)$.

Remarque 3.16. - Dans la suite, on se fixera donc $\mathscr{V}$ un anneau de Cohen associé à $k$ et on fera des extensions des scalaires si on veut travailler sur une extension de $K=\operatorname{Frac}(\mathscr{V})$. On notera parfois $H_{\text {rig }}^{i}(X)\left(\right.$ resp. $\left.H_{Z \text {,rig }}^{i}(X)\right)$ les groupes de cohomologie $H_{\text {rig }}^{i}(X / K)$ (resp. $\left.H_{Z \text {,rig }}^{i}(X / K)\right)$ quand il n'y aura pas d'ambiguïté.

On a aussi :

Proposition 3.17 (multiplicativité). - Soit $Z$ un fermé de $X$ qui est localement les zéros d'un élément de $\mathscr{O}_{X}$, le morphisme

$$
c_{1}: \operatorname{Div}_{Z}(X) \longrightarrow H_{Z, \text { rig }}^{2}(X / K)
$$

est un morphisme de groupe.

TOME $131-2003-\mathrm{N}^{\mathrm{O}} 1$ 
Démonstration. - On se donne $D=(\mathscr{L}, Z, s)$ et $D^{\prime}=\left(\mathscr{L}^{\prime}, Z, s^{\prime}\right)$ deux éléments de $\operatorname{Div}_{Z}(X)$. Avec les notations précédentes, on peut choisir un recouvrement affine $\mathfrak{U}$ de $\mathscr{Y}$ dont la restriction à $X$ trivialise $\mathscr{L}$ et $\mathscr{L}^{\prime}$ et tel que $Z_{i}$ soit le complémentaire d'une section $f_{i}$ dans $X_{i}$ pour tout $i$. Dès lors, en choisissant des trivialisations $\varphi_{i}: \mathscr{O}_{X_{i}} \rightarrow \mathscr{L}_{\mid X_{i}}\left(\right.$ resp. $\left.\varphi_{i}^{\prime}: \mathscr{O}_{X_{i}} \rightarrow \mathscr{L}_{\mid X_{i}}^{\prime}\right)$ de $\mathscr{L}$ (resp. $\mathscr{L}^{\prime}$ ) compatibles à $s$ (resp. $s^{\prime}$ ), on obtient une trivialisation de $\mathscr{L} \otimes \mathscr{L}^{\prime}$ compatible à $s \otimes s^{\prime}$ en considérant $\varphi \otimes \varphi^{\prime}$. En reprenant la construction de $c_{1}$ et en utilisant

$$
\frac{\mathrm{d}\left(u \cdot u^{\prime}\right)}{\left(u \cdot u^{\prime}\right)}=\frac{\mathrm{d} u}{u}+\frac{\mathrm{d} u^{\prime}}{u^{\prime}}, \quad \log ((1+u)(1+v))=\log (1+u)+\log (1+v),
$$

on montre notre proposition.

Nous allons conclure ces propriétés en établissant que notre classe est compatible à l'extension du support. C'est cette propriété qui nous permettra de traiter le cas des variétés ouvertes.

Soient $D=(\mathscr{L}, Z, s)$ un bon pseudo-diviseur et $Z^{\prime}$ un fermé de $X$ qui est localement l'ensemble des zéros d'une section de $\mathscr{O}_{X}$ et qui contient $Z$. On regarde le pseudo-diviseur $D^{\prime}=\left(\mathscr{L}, Z^{\prime}, s^{\prime}\right)$ où $s^{\prime}$ est la restriction de $s$ à $X-Z^{\prime}$. On a la proposition :

Proposition 3.18. - Avec les notations précédentes, le morphisme canonique

envoie $c_{1}(D)$ sur $c_{1}\left(D^{\prime}\right)$.

$$
H_{Z, \text { rig }}^{2}(X / K) \longrightarrow H_{Z^{\prime}, \text { rig }}^{2}(X / K)
$$

Démonstration. - On se donne un plongement $X \hookrightarrow \mathscr{Y}$ dans $\mathscr{V}$-schéma formel lisse au voisinage de $X$, un recouvrement affine $\mathfrak{U}$ de $\mathscr{Y}$ dont la restriction $\mathfrak{U}_{X}$ à $X$ induit une trivialisation de $\mathscr{L}$ compatible à $s$ (et donc à $s^{\prime}$ ). On peut donc prendre le même cocycle $(u)$ pour calculer $c_{1}(D)$ et $c_{1}\left(D^{\prime}\right)$. Les deux termes à valeurs dans le complexe de de Rham de $] X$ [ sont donc les mêmes. Il reste donc à comparer les termes surconvergents. Il suffit alors de voir que, comme $X_{i}-Z_{i}^{\prime} \subset X_{i}-Z_{i}$, les fonctions surconvergentes le long de $Z_{i}$ dans le calcul de $c_{1}(D)$ sont aussi surconvergentes le long de $Z_{i}^{\prime}$. On peut donc ainsi calculer $c_{1}\left(D^{\prime}\right)$.

3.2. Cas des variétés ouvertes. - Nous allons voir comment, par fonctorialité, nous pouvons généraliser la classe d'un pseudo-diviseur au cas des variétés non nécessairement propres. La méthode consiste à compactifier la variété de départ de telle sorte que l'on puisse prolonger le pseudo-diviseur au compactifié. La classe du pseudo-diviseur s'obtient alors par fonctorialité.

Lemme 3.19. - Soit $X$ une $k$-variété, il existe une $k$-variété propre $\bar{X}$ et une immersion ouverte $j: X \hookrightarrow \bar{X}$ telles que $Z=\bar{X}-X$ soit un diviseur. 
Démonstration. - D'après Nagata [27], il existe une $k$-variété propre $\bar{X}_{1}$ et une immersion ouverte $j_{1}: X \hookrightarrow \bar{X}_{1}$. Si on note $Z_{1}=\bar{X}_{1}-X$, on considère l'éclatement $\bar{X}$ de $\bar{X}_{1}$ le long de $Z_{1}$. C'est encore une $k$-variété propre. De plus, l'éclatement ne modifiant pas $\bar{X}_{1}-Z_{1}, j_{1}$ se prolonge en une immersion ouverte $j: X \hookrightarrow \bar{X}$ et $Z=\bar{X}-X$ est un diviseur.

Remarque 3.20. - Par la suite, nous noterons $(\bar{X}, j)$ la donnée consistant en la variété propre $\bar{X}$ et l'immersion ouverte $j: X \hookrightarrow \bar{X}$. Nous supposerons toujours - sauf mention explicite — que nos compactifications vérifient la condition du lemme 3.19 .

Proposition 3.21. - Soient $X$ une k-variété et $D=(\mathscr{L}, Z, s)$ un bon pseudo-diviseur. Il existe une compactification de $X, j: X \hookrightarrow \bar{X}$ et $\bar{D}=(\overline{\mathscr{L}}, \bar{Z}, \bar{s})$ un bon pseudo-diviseur sur $\bar{X}$ tel que

$$
j^{*}(\bar{D})=D \text {. }
$$

Démonstration. - Il suffit de montrer qu'il existe une compactification $(\bar{X}, j)$ et un faisceau inversible $\overline{\mathscr{L}}$ sur $\bar{X}$ tels que $j^{*} \overline{\mathscr{L}}=\mathscr{L}$. En effet supposons que l'on ait une telle donnée. On pose $\bar{Z}=\bar{X}-(X-Z)=Z \cup(\bar{X}-X)$, alors $\bar{X}-\bar{Z}=X-Z$ et on peut prendre $\bar{s}=s$. Comme $\bar{X}-X$ est un diviseur, $\bar{D}=(\overline{\mathscr{L}}, \bar{Z}, \bar{s})$ est bien un bon pseudo-diviseur.

On se donne une compactification $\left(\bar{X}_{1}, j_{1}\right)$ de $X$. D'après [EGAI, 6.9.8], on sait qu'il existe $\mathscr{L}_{1}$ un sous-faisceau cohérent de $j_{1 *}(\mathscr{L})$ tel que $j_{1}^{*}\left(\mathscr{L}_{1}\right)=\mathscr{L}$. Dès lors, on regarde les fibrés projectifs

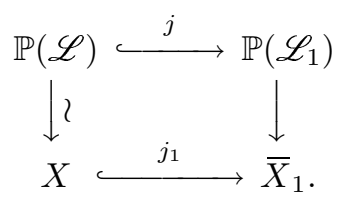

On a alors $j^{*}\left(\mathscr{O}_{\mathbb{P}\left(\mathscr{L}_{1}\right)}(1)\right)=\mathscr{O}_{\mathbb{P}(\mathscr{L})}(1)$.

Remarque 3.22. - En itérant cette méthode, si on se donne $D_{1}, \ldots, D_{n}$ des pseudo-diviseurs sur $X$, on peut trouver une compactification $(\bar{X}, j)$ et des pseudo-diviseurs $\bar{D}_{1}, \ldots, \bar{D}_{n}$ sur $\bar{X}$ tels que pour tout $i$ on ait

$$
j^{*} \bar{D}_{i}=D_{i} .
$$

Notons que ce théorème admet une généralisation, en remplaçant les pseudodiviseurs par des faisceaux localement libres (voir théorème 5.32), qui nous sera utile lors de l'étude des classes de Chern.

On va définir la classe d'un pseudo-diviseur $D$ comme l'image par le morphisme de fonctorialité de la classe d'un prolongement de $D$ sur une compactification. Pour cela, nous avons besoin du théorème suivant.

TOME $131-2003-\mathrm{N}^{\mathrm{O}} 1$ 
Proposition 3.23. - Soient $X$ une variété sur $k$ et $D$ un pseudo-diviseur sur $X$. Pour $\ell \in\{1,2\}$ on se donne $\left(\bar{X}_{\ell}, j_{\ell}\right)$ une compactification de $X$ et $\bar{D}_{\ell}$ un pseudo-diviseur prolongeant $D$. On a alors

$$
j_{1 \text { rig }}^{*}\left(c_{1}\left(\bar{D}_{1}\right)\right)=j_{2 \text { rig }}^{*}\left(c_{1}\left(\bar{D}_{2}\right)\right),
$$

où $j_{1}{ }_{\text {rig }}^{*}$ et $j_{2 \text { rig }}^{*}$ sont les morphismes de fonctorialités.

Démonstration. - Nous allons d'abord nous ramener à deux prolongements de notre pseudo-diviseur sur la même variété. Nous utiliserons pour cela la méthode habituelle du plongement diagonal. On a le diagramme suivant

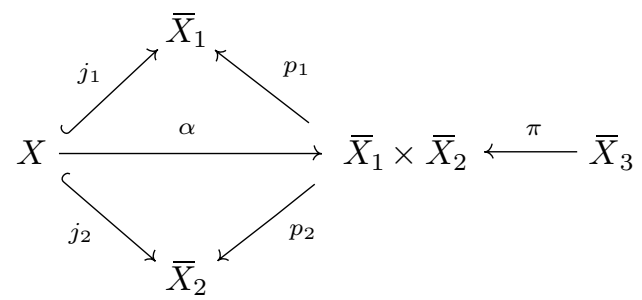

où $\pi$ est l'éclatement de l'adhérence schématique de $X$ dans $\bar{X}_{1} \times \bar{X}_{2}$ en le complémentaire de $X$. Les fonctorialités des classes associées aux pseudo-diviseurs dans le cas propre nous ramènent à montrer notre théorème pour $\left(p_{1} \circ \pi\right)^{*}\left(\bar{D}_{1}\right)$ et $\left(p_{2} \circ \pi\right)^{*}\left(\bar{D}_{2}\right)$, tous deux pseudo-diviseurs sur $\bar{X}_{3}$. En effet, si on note encore $\alpha$ l'inclusion de $X$ dans $\bar{X}_{3}$, on a pour tout $\ell \in\{1,2\}$

$$
j_{\ell_{\text {rig }}^{*}}\left(c_{1}\left(\bar{D}_{\ell}\right)\right)=\alpha^{*}\left(p_{\ell} \circ \pi\right)^{*}\left(c_{1}\left(\bar{D}_{\ell}\right)\right)=\alpha^{*} c_{1}\left(\left(p_{\ell} \circ \pi\right)^{*} \bar{D}_{\ell}\right) .
$$

De plus, grâce à la proposition 3.17 , on est ramené à montrer que si on a une compactification $(\bar{X}, j)$ et un pseudo-diviseur $\bar{D}=(\overline{\mathscr{L}}, \bar{Z}, \bar{s})$ sur $\bar{X}$ tel que $j^{*} \overline{\mathscr{L}}=\mathscr{O}_{X}$, alors

$$
j_{\text {rig }}^{*} c_{1}(\bar{D})=0 \quad \text { dans } \quad H_{Z, \text { rig }}^{2}(X)
$$

où $Z=\bar{Z} \cap X$. Maintenant, notons $T=\bar{X}-X$. On sait que le faisceau $\mathscr{L}$ admet une section inversible sur $X$ entier (et non seulement $X-Z$ ). Donc $\bar{D}$ est obtenu par extension du support à partir d'un pseudo-diviseur de support $T$. L'équation (3) découle donc de la suite exacte d'excision (proposition 1.3)

$$
\cdots \rightarrow H_{T, \text { rig }}^{2}(\bar{X}) \longrightarrow H_{\bar{Z}, \text { rig }}^{2}(\bar{X}) \stackrel{j^{*}}{\longrightarrow} H_{Z, \text { rig }}^{2}(X) \rightarrow \cdots
$$

et de la compatibilité de nos classes à l'extension du support.

On a donc défini des classes de cohomologie associées à un pseudo-diviseur sur $X$. En utilisant la remarque à la proposition 3.21 , on voit que la multiplicativité de ces classes se déduit du cas propre :

Proposition 3.24 (multiplicativité). - Soit $Z$ un fermé de $X$, le morphisme $c_{1}: \operatorname{Div}_{Z}(X) \rightarrow H_{Z, \text { rig }}^{2}(X / K)$ est un morphisme de groupe.

BULletin DE LA SOCIÉtÉ MATHÉMATIQUE DE FRANCE 
Afin de ramener la démonstration de la fonctorialité de ces classes au cas propre, nous allons avoir besoin d'une variante de la proposition 3.21.

Proposition 3.25. - Soient $X, X^{\prime}$ deux k-variétés et un morphisme $f$ : $X^{\prime} \rightarrow X$. Il existe des compactifications $(\bar{X}, j)$ et $\left(\bar{X}^{\prime}, j^{\prime}\right)$ de $X$ et $X^{\prime}$ respectivement, telles qu'on puisse trouver un morphisme propre $\bar{f}: \bar{X}^{\prime} \rightarrow \bar{X}$ de sorte que l'on ait le diagramme commutatif :

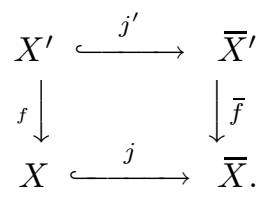

De plus, si on se donne un pseudo-diviseur D sur $X$, on peut choisir ces compactifications de sorte qu'il existe un pseudo-diviseur $\bar{D}$ sur $\bar{X}$ tel que

$$
j^{*}(\bar{D})=D .
$$

On a alors

$$
\left(j^{\prime}\right)^{*}\left(\bar{f}^{*} \bar{D}\right)=f^{*} D
$$

Démonstration. - On se donne des compactifications $(\bar{X}, j)$ et $\left(\bar{X}_{1}^{\prime}, j^{\prime}\right)$ de $X$ et $X^{\prime}$ respectivement. On regarde alors $\bar{X}_{2}^{\prime}$ l'adhérence schématique de $X^{\prime}$ dans $\bar{X} \times \bar{X}_{1}^{\prime}$. On note alors $\bar{X}^{\prime}$ la variété obtenue en éclatant le complémentaire de $X^{\prime}$ dans $\bar{X}_{2}^{\prime}$. L'éclatement ayant lieu en dehors de $X^{\prime}$, l'immersion de $X^{\prime}$ dans $\bar{X}_{2}^{\prime}$ se relève en une immersion de $X^{\prime}$ dans $\bar{X}^{\prime}$.

On a donc le diagramme commutatif

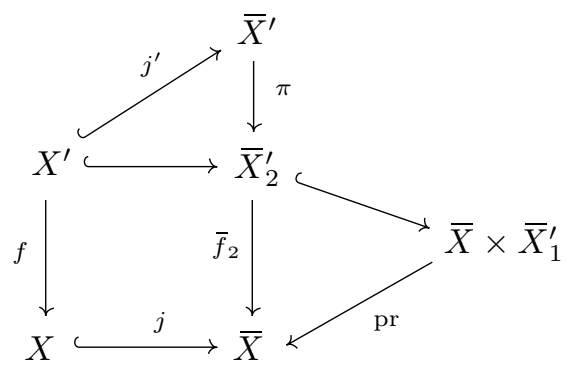

où pr désigne la projection sur le premier terme. La flèche $\bar{f}=\pi \circ \bar{f}_{2}$ est donc propre comme composition de morphismes propres. De plus, si on se donne sur $X$ un pseudo-diviseur $D$, on peut grâce à la proposition 3.21 choisir la compactification $(\bar{X}, j)$ pour qu'il existe sur $\bar{X}$ un pseudo-diviseur $\bar{D}$ vérifiant $j^{*} \bar{D}=D$. La dernière propriété est formelle.

On en déduit la proposition suivante :

TOME $131-2003-\mathrm{N}^{\mathrm{O}} 1$ 
Proposition 3.26 (fonctorialité). - Soient $f: X^{\prime} \rightarrow X$ un morphisme de variété et $D$ un bon pseudo-diviseur $D=(\mathscr{L}, Z, s)$. Dans $H_{f^{-1}(Z) \text {,rig }}^{2}(X)$, on a l'égalité

$$
f^{*} c_{1}(D)=c_{1}\left(f^{*} D\right)
$$

\section{Classes de Chern}

Dans cette section, on se donne $X$ une $k$-variété.

DÉfinition 4.1 (première classe de Chern). - Soit $\mathscr{L}$ un faisceau inversible sur $X$. On appelle première classe de Chern de $\mathscr{L}$ et on note $c_{1}(\mathscr{L})$ l'élément de $H_{\text {rig }}^{2}(X)$ défini par

$$
c_{1, \text { rig }}(\mathscr{L}):=c_{1}((\mathscr{L}, X,-))
$$

où le terme de droite est la classe de cohomologie associée au pseudo-diviseur $(\mathscr{L}, X,-)$.

REMARque 4.2. - Si $X$ est une variété propre, on peut reprendre la construction de la partie 3 dans le cas particulier où le support est $X$. À ce moment là, on retrouve une construction similaire à la construction classique des classes de Chern en cohomologie de de Rham.

Ainsi définie, la première classe de Chern hérite des propriétés des classes des pseudo-diviseurs : fonctorialité, multiplicativité, compatibilité à l'extension des scalaires.

Nous allons suivre la méthode classique [18] afin de construire les autres classes de Chern d'un faisceau localement libre.

Nous allons calculer la cohomologie rigide d'un fibré projectif relatif. Plus précisément on se donne $X$ une $k$-variété et $\mathscr{E}$ un faisceau localement libre de rang $r$. On note $\mathbb{P}=\mathbb{P}(\mathscr{E})$ le fibré projectif associé, $p: \mathbb{P} \rightarrow X$ la projection et

$$
\xi=c_{1, \text { rig }}\left(\mathscr{O}_{\mathbb{P}}(1)\right) \in H_{\text {rig }}^{2}(\mathbb{P} / K) .
$$

On peut alors regarder $\xi$ comme un morphisme dans $D^{+}\left(X_{\mathrm{Zar}}\right)$ :

$$
\xi: \mathbb{Z}_{X} \longrightarrow \mathbb{R} p_{*} \mathbb{R}_{\text {rig }}(\mathbb{P} / K)[2],
$$

où $\mathbb{Z}_{X}$ est le faisceau constant $\mathbb{Z}$ sur $X$. Par cup-produit, on a alors

$$
\xi^{i}: \mathbb{Z}_{X} \longrightarrow \mathbb{R} p_{*} \mathbb{R} \underline{\Gamma}_{\text {rig }}(\mathbb{P} / K)[2 i]
$$

Enfin, on a le morphisme de fonctorialité

$$
\mathbb{R} \underline{\Gamma}_{\text {rig }}(X / K) \longrightarrow \mathbb{R} p_{*} \mathbb{R} \underline{\Gamma}_{\text {rig }}(\mathbb{P} / K) .
$$

On en déduit alors le morphisme dans $D^{+}\left(X_{\text {Zar }}\right)$ :

$$
\bigoplus_{i=0}^{r-1} \xi^{i}: \bigoplus_{i=0}^{r-1} \mathbb{R} \underline{\Gamma}_{\text {rig }}(X / K)[-2 i] \longrightarrow \mathbb{R} p_{*} \mathbb{R} \underline{\Gamma}_{\text {rig }}(\mathbb{P} / K) \text {. }
$$

BULLETIN DE LA SOCIÉtÉ MATHÉMATIQUE DE FRANCE 
Proposition 4.3. - Avec les notations précédentes, l'application (4) est un isomorphisme.

Démonstration. - Le principe de la démonstration est classique. La question étant locale sur $\bar{X}$, on peut supposer que $\mathscr{E}=\mathscr{O}_{X}^{r}$. On se donne une immersion fermée $\bar{X} \hookrightarrow \mathscr{Y}$ dans un $\mathscr{V}$-schéma formel lisse. On a alors le diagramme commutatif suivant :

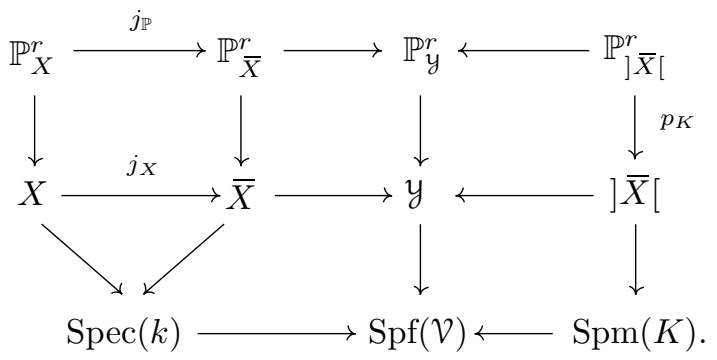

Le faisceau $\mathscr{O}_{\mathbb{P}_{X}^{r}}(1)$ est la restriction du faisceau $\mathscr{O}_{\mathbb{P}_{X}^{r}}(1)$ qui se relève en $\mathscr{O}_{\mathbb{P}_{\mathscr{Y}}^{r}}(1)$. Dans ce cas, on a vu (remarque 3.11), que si $\mathfrak{U}$ est un recouvrement de $\mathbb{P}_{\mathscr{Y}}^{r}$ trivialisant $\mathscr{O}_{\mathbb{P}_{\mathscr{O}}^{r}}(1)$ et $(u) \in Z^{1}\left(\mathfrak{U}, \mathscr{O}_{\mathscr{Y}}^{*}\right)$ est un cocycle représentant le faisceau $\mathscr{O}_{\mathbb{P}_{\mathscr{Y}}^{r}}(1)$, alors la classe de Chern de $\mathscr{O}_{\mathbb{P}_{X}^{r}}(1)$ est calculée par le cocycle

$$
\left(\frac{\mathrm{d} u}{u}\right) \in Z^{2}\left(\mathfrak{U}_{K}, \Omega_{] \bar{X}[}^{*}\right) .
$$

Ce dernier, étant le cocyle qui calcule la première classe de Chern en cohomologie de de Rham du faisceau $\mathscr{O}_{\mathbb{P}^{r} \bar{X}[}(1)$. On est ramené à montrer que l'application

$$
\bigoplus_{i=0}^{r-1} j_{X}^{\dagger} \Omega_{] \bar{X}[}^{\star}[-2 i] \longrightarrow \mathbb{R} p_{K_{*}} j_{\mathbb{P}}^{\dagger} \Omega_{] \mathbb{P}_{\bar{X}}[}^{\star}
$$

définie par la restriction de la première classe de Chern en cohomologie de de Rham est un isomorphisme. Il suffit alors de remarquer que si on se donne une famille cofinale de voisinages stricts de $] X[$ dans $] \bar{X}$ [, disons $\left(V_{i}\right)_{i \in I}$, la famille des $\mathbb{P}_{V_{i}}^{r}$ est une famille cofinale de voisinages stricts de $\mathbb{P}_{] X[}^{r}$ dans $\mathbb{P}_{] \bar{X}}^{r}$. Le morphisme $p_{i, K}: \mathbb{P}_{V_{i}}^{r} \rightarrow V_{i}$ étant quasi-compact et quasi-séparé il commute avec les limites inductives filtrantes [4, 0.1.8]. Il reste donc à montrer que

$$
\bigoplus_{i=0}^{r-1} \Omega_{V_{i}}^{\star}[-2 i] \longrightarrow \mathbb{R} p_{i, K_{*}} \Omega_{\mathbb{P}_{V_{i}}}^{\star}
$$

est un isomorphisme. On peut alors se réduire au cas où $V_{i}$ est un affinoïde.

Ce résultat se déduit alors d'un théorème de type GAGA relatif en géométrie rigide [25, 2.8] et du résultat similaire en cohomologie de de Rham algébrique [20].

TOME $131-2003-\mathrm{N}^{\mathrm{O}} 1$ 
Corollaire 4.4. - Avec les notations ci-dessus, on a pour tout $n$ la décomposition suivante:

$$
H_{\text {rig }}^{n}(\mathbb{P} / K)=\bigoplus_{i=0}^{r-1} H_{\text {rig }}^{n-2 i}(X / K) \cdot \xi^{i} .
$$

On applique la décomposition du corollaire 4.4 à $\xi^{r}$ et on obtient, comme dans le cas classique,

$$
\xi^{r}=\sum_{i=1}^{r}(-1)^{i+1} c_{i}(\mathscr{E}) \xi^{r-i},
$$

avec $c_{i}(\mathscr{E}) \in H_{\text {rig }}^{2 i}(X / K)$.

DÉfinition 4.5. - Avec les notations précédentes, pour $0<i \leqslant r$, on appelle $i$-ème classe de Chern de $\mathscr{E}$ la classe $c_{i}(\mathscr{E})$. On pose de plus

$$
c_{0}(\mathscr{E})=1 \quad \text { dans } \quad H_{\text {rig }}^{0}(X / K) .
$$

REMARque 4.6. - En appliquant ce que l'on vient de voir à un fibré inversible $\mathscr{F}$, on retrouve notre première classe de Chern. En effet, la décomposition (5) devient alors

$$
c_{1, \operatorname{rig}}\left(\mathscr{O}_{\mathbb{P}(\mathscr{F})}(1)\right)=p^{*} c_{1}(\mathscr{F}) .
$$

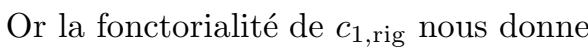

$$
c_{1, \text { rig }}\left(\mathscr{O}_{\mathbb{P}(\mathscr{F})}(1)\right)=p^{*} c_{1, \text { rig }}(\mathscr{F}) .
$$

On conclut en utilisant que $p^{*}$ est injectif.

Proposition 4.7 (fonctorialité). — Soient $X$ et $X^{\prime}$ deux $k$-variétés propres et $f: X^{\prime} \rightarrow X$ un morphisme. Pour tout $\mathscr{E}$ faisceau localement libre de rang $r$ sur $X$ et tout $i$, on a

$$
c_{i}\left(f^{*} \mathscr{E}\right)=f^{*} c_{i}(\mathscr{E}) .
$$

Démonstration. — On note $\mathbb{P}^{\prime}=\mathbb{P}\left(f^{*}(\mathscr{E})\right)$ et $\xi^{\prime}=c_{1}\left(\mathscr{O}_{\mathbb{P}^{\prime}}(1)\right)$. La fonctorialité de la première classe de Chern nous assure que

$$
\xi^{\prime}=f^{*} \xi
$$

On applique alors $f^{*}$ à la décomposition (5). Le morphisme $f^{*}$ étant compatible au produit, on obtient dans $H_{\text {rig }}^{2 r}\left(\mathbb{P}^{\prime} / K\right)$ :

$$
\xi^{\prime r}=\sum_{i=1}^{r}(-1)^{i} f^{*} c_{i}(\mathscr{E}) \xi^{\prime r-i}
$$

La décomposition étant unique, on a

$$
c_{i}\left(f^{*} \mathscr{E}\right)=f^{*} c_{i}(\mathscr{E}) .
$$


REMARQUE 4.8. - La définition utilisée pour la cohomologie rigide ne permet pas d'utiliser la méthode classique [8], [14] pour démontrer l'additivité des classes de Chern. En effet, de par la définition de la cohomologie rigide basé sur des choix de plongements, il semble difficile de définir la cohomologie rigide des topos classifiants et des variétés simpliciales dont on a besoin. Nous différons donc la démonstration de cette propriété à la section suivante.

\section{Comparaison avec la cohomologie cristalline et additivité}

Après avoir fait quelques rappels sur les topos cristallins de niveau $m$, nous construisons des classes de Chern à valeurs dans ces derniers en nous inspirant du cas cristallin classique (de niveau 0) [8]. Cela nous permettra, pour $X$ propre, de donner une construction cristalline des classes de Chern rigides construites précédemment. Cette formulation permet d'appliquer la méthode classique [14], [8] pour démontrer l'additivité des classes de Chern en se ramenant à une situation universelle.

On établira aussi un théorème de comparaison avec les classes de Chern à valeur dans le topos convergent [28].

5.1. Les topos $\boldsymbol{m}$-cristallins. - Nous allons énoncer les principaux résultats sur la cohomologie cristalline de niveau $m$. Pour de plus amples informations on pourra consulter [34].

On fixe une fois pour toutes un nombre premier $p$. Commençons par rappeler la définition d'un $m$-PD-idéal et de l'enveloppe à puissances divisées partielles de niveau $m$. On trouvera un exposé détaillé de cette théorie dans [5, 1].

DÉfinition 5.1. - Soient $A$ une $\mathbb{Z}_{(p)}$-algèbre, $I \subset A$ un idéal et $m \geqslant 0$ un entier. On appelle structure partielle d'idéal à puissances divisées de niveau $m$ sur $I$ ( $m$-PD-structure) la donnée d'un PD-idéal $(J, \gamma) \subset I$ tel que

$$
I^{\left(p^{m}\right)}+p I \subset J
$$

Proposition 5.2. - Soient $A$ une $\mathbb{Z}_{(p)}$-algèbre et $I \subset A$ un idéal quelconque. Il existe une A-algèbre $P^{m}(I)$ et un $m$-PD-idéal $\bar{I} \subset P^{m}(I)$ tel que $I P^{m}(I) \subset \bar{I}$, qui soient universels pour les morphismes de $A$ dans un anneau $A^{\prime}$ envoyant $I$ dans un $m$-PD-idéal $I^{\prime}$.

DÉfinition 5.3. - L'algèbre $P^{m}(I)$ munie de son $m$-PD-idéal $\bar{I}$ est appelée l'enveloppe à puissances divisées partielles de niveau $m$ de $(A, I)$.

Remarque 5.4. - L'idéal (0) étant un $m$ PD-idéal pour tout $m$, la propriété universelle de l'enveloppe à puissances divisées appliquée au morphisme

TOME $131-2003-\mathrm{N}^{\mathrm{O}} 1$ 
$A \rightarrow A / I$ donne le diagramme commutatif suivant :

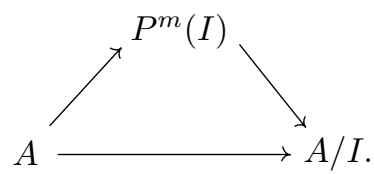

Soit $C$ un anneau de Cohen pour $k$. On rappelle [1, I.1.2.4] qu'il existe une unique structure de PD-idéal sur l'idéal maximal de $C$ notée $\gamma$. On note $S=\operatorname{Spf}(C)$. Dans toute la suite, nous ne regarderons que les topos cristallins de base $S$ car ce sont eux qui interviennent dans la comparaison avec la cohomologie rigide.

DÉfinition 5.5. - Soient $X$ un $S$-schéma sur lequel $p$ est localement nilpotent et $m \in \mathbb{N}$. On appelle site cristallin de niveau $m$ de $X$ relativement $\grave{a}$ $(S,(p), \gamma)$ et on note $\mathrm{Cris}^{m}(X / S)$ le site ayant pour objets les $S$-immersions fermées $U \hookrightarrow T$ où $U$ est un ouvert de $X, p$ est localement nilpotent sur $T$ et où l'idéal définissant ces immersions est muni d'une $m$-PD-structure compatible à $\gamma$.

Par la suite nous appellerons topos cristallin de niveau $m$ et nous noterons $(X / S)_{\text {cris }}^{m}$ le topos associé au site $\operatorname{Cris}^{m}(X / S)$.

Remarque 5.6. - Pour $m=0$, on retrouve le topos cristallin classique.

Il est possible de donner une description des objets du topos cristallin de niveau $m$ similaire à celle que l'on a pour le topos cristallin classique. Pour définir un faisceau $\mathscr{F}$ du topos cristallin de niveau $m$, il suffit de donner pour tout élément $(U, T)$ du site $m$-cristallin, un faisceau zariskien $\mathscr{F}_{(U, T)}$ (noté aussi $\left.\mathscr{F}_{T}\right)$ sur $T$; ces données étant assujetties à des conditions de compatibilités similaires à celles du cas classique.

On peut de cette manière construire les faisceaux suivants :

- Le faisceau $\mathscr{O}_{X / S}^{m}$ est défini par

$$
\left(\mathscr{O}_{X / S}^{m}\right)_{(U, T)}=\mathscr{O}_{T} .
$$

- Le faisceau $\mathscr{I}_{X / S}^{m}$ est défini par

$$
\left(\mathscr{I}_{X / S}^{m}\right)_{(U, T)}=\mathscr{I}_{T}
$$

où $\mathscr{I}_{T}$ est le faisceau définissant l'immersion $U \hookrightarrow T$.

- Le faisceau $\mathscr{J}_{X / S}^{m}$ est défini par

$$
\left(\mathscr{J}_{X / S}^{m}\right)_{(U, T)}=\mathscr{J}_{T}
$$

où $\mathscr{J}_{T}$ est le PD-idéal définissant la $m$-PD structure sur $\mathscr{I}_{T}$.

BULLETIN DE LA SOCiÉtÉ MATHÉMATIQUE DE FRANCE 
Notations 5.7. - Pour tout $k$-variété $X$ et tout $m$, on note

$$
H_{m \text {-cris }}^{i}(X):=H^{i}\left((X / S)_{\text {cris }}^{m}, \mathscr{O}_{X / S}^{m}\right) .
$$

Nous allons maintenant étudier comment se comportent ces topos quand on fait varier $m$.

Proposition 5.8. - Pour tous $m, m^{\prime}$ avec $m \leqslant m^{\prime}$, il existe un morphisme de topos

$$
i_{m^{\prime}, m}:(X / S)_{\text {cris }}^{m} \longrightarrow(X / S)_{\text {cris }}^{m^{\prime}}
$$

De plus ces morphismes vérifient que pour tout triplet $m \leqslant m^{\prime} \leqslant m^{\prime \prime}$, on a

$$
i_{m^{\prime \prime}, m} \stackrel{\sim}{\longrightarrow} i_{m^{\prime \prime}, m^{\prime}} \circ i_{m^{\prime}, m} .
$$

Ces morphismes sont donnés de la manière suivante :

- Pour tout $\mathscr{E}^{\prime}$ dans $(X / S)_{\text {cris }}^{m^{\prime}}$ et tout $(U, T, \mathscr{I}) \in \operatorname{Cris}^{m}(X / S)$, on a

$$
\Gamma\left((U, T), i_{m^{\prime}, m}^{*} \mathscr{E}^{\prime}\right)=\Gamma\left((U, T), \mathscr{E}^{\prime}\right)
$$

en remarquant que l'idéal $\mathscr{J}$ définissant la $m$-PD-structure de $\mathscr{I}$ définit aussi une $m^{\prime}$-PD-structure sur $\mathscr{I}$.

- Pour tout $\mathscr{E}$ dans $(X / S)_{\text {cris }}^{m}$ et tout $(U, T, \mathscr{I}) \in \operatorname{Cris}^{m^{\prime}}(X / S)$, on a

$$
\Gamma\left((U, T), i_{m^{\prime}, m_{*}} \mathscr{E}\right)=\Gamma\left(\left(U, T^{m}\right), \mathscr{E}\right),
$$

où $T^{m}$ est l'enveloppe à puissances divisées de niveau $m$ de $\mathscr{I}$ compatible aux puissances divisées définissant la $m^{\prime}$-PD-structure de $\mathscr{I}$.

On construit alors un morphisme canonique [3]

$$
u_{m^{\prime}, m}: \mathscr{O}_{X / S}^{m^{\prime}} \longrightarrow i_{m^{\prime}, m *} \mathscr{O}_{X / S}^{m}
$$

donné sur un épaississement $(U, T)$ par le morphisme canonique $\mathscr{O}_{T} \rightarrow \mathscr{O}_{T^{m}}$. Le morphisme $i_{m^{\prime}, m}$ devient ainsi un morphisme de topos annelés.

Pour finir avec ces généralités sur les topos $m$-cristallins, notons qu'il existe, comme dans le cas classique, un morphisme de projection sur le topos zariskien

$$
u_{X / S}^{m}:(X / S)_{\text {cris }}^{m} \longrightarrow X_{\text {Zar }},
$$

ainsi qu'un morphisme d'inclusion du topos zariskien dans le topos $m$-cristallin :

$$
i_{X / S}^{m}: X_{\mathrm{Zar}} \longrightarrow(X / S)_{\mathrm{cris}}^{m} \text {. }
$$

Lemme 5.9. - Pour tous $m, m^{\prime}$ avec $m \leqslant m^{\prime}$, on a les diagrammes commutatifs suivants :

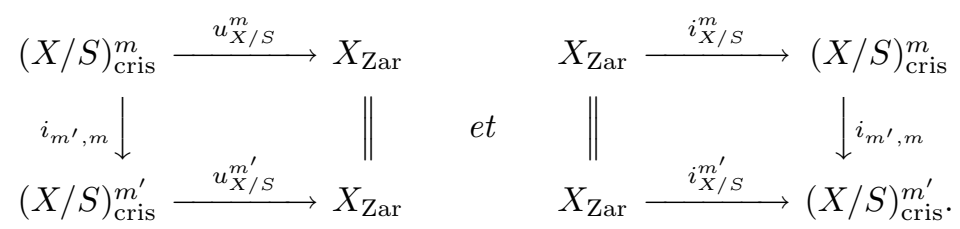

TOME $131-2003-\mathrm{N}^{\mathrm{O}} 1$ 
5.2. Classes de Chern $\boldsymbol{m}$-cristallines. - Nous allons généraliser la construction de [8] au cas des topos $m$-cristallins. On se fixe $m \in \mathbb{N}$; avec les notations précédentes, on a, comme dans le cas de la cohomologie cristalline de niveau 0 , une suite exacte dans $(X / S)_{\text {cris }}^{m}$ :

$$
0 \rightarrow 1+\mathscr{I}_{X / S}^{m} \longrightarrow\left(\mathscr{O}_{X / S}^{m}\right)^{*} \longrightarrow i_{*}^{m}\left(\mathscr{O}_{X}^{*}\right) \rightarrow 0 .
$$

On considère le cobord de cette suite exacte qui nous fournit un morphisme dans $D^{+}\left((X / S)_{\text {cris }}^{m}, \mathrm{Ab}\right)$ :

$$
i_{*}^{m}\left(\mathscr{O}_{X}^{*}\right)[1] \rightarrow\left(1+\mathscr{I}_{X / S}^{m}\right)[2] .
$$

L'élévation à la puissance $p^{m}$ nous donne un morphisme :

$$
1+\mathscr{I}_{X / S}^{m} \longrightarrow 1+\mathscr{J}_{X / S}^{m} \text {. }
$$

L'idéal $\mathscr{J}_{X / S}^{m}$ est muni d'une structure de PD-idéal, on peut donc construire un logarithme. En le composant avec le morphisme précédent, on obtient un morphisme

$$
\begin{aligned}
& \psi_{m}: 1+\mathscr{I}_{X / S}^{m} \longrightarrow \mathscr{J}_{X / S}^{m}, \\
& (1+x) \longmapsto \log \left((1+x)^{p^{m}}\right) .
\end{aligned}
$$

En composant (6) avec $\psi_{m}$, on obtient

$$
c_{1, m}: i_{*}^{m}\left(\mathscr{O}_{X}^{*}\right)[1] \longrightarrow \mathscr{J}_{X / S}^{m}[2] .
$$

Par suite, en composant avec $\mathscr{J}_{X / S}^{m} \rightarrow \mathscr{O}_{X / S}^{m}$ et en passant à la cohomologie, on a

$$
c_{1, m}: H^{1}\left(X, \mathscr{O}_{X}^{*}\right) \longrightarrow H_{m-\operatorname{cris}}^{2}(X) .
$$

Proposition 5.10 (fonctorialité). - Soient $X$ et $Y$ deux $k$-variétés, $f: X \rightarrow Y$ un morphisme et $\mathscr{F}$ un faisceau inversible sur $Y$; on a

$$
c_{1, m}\left(f^{*} \mathscr{F}\right)=f^{*} c_{1, m}(\mathscr{F}) .
$$

Démonstration. - La preuve découle directement du diagramme commutatif

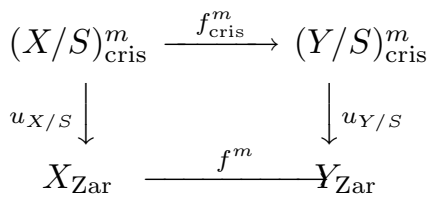

et du fait que les suites exactes utilisées sont fonctorielles.

Maintenant que l'on a construit la première classe de Chern d'un faisceau inversible, nous allons en déduire les autres par la méthode habituelle [18].

On se donne $\mathscr{E}$ un faisceau localement libre de rang $r$ sur $X$, on note $\mathbb{P}=\mathbb{P}(\mathscr{E})$ son fibré projectif et $\mathscr{O}_{\mathbb{P}}(1)$ le faisceau canonique sur ce dernier. Le morphisme structural de $\mathbb{P}$ sera noté $p: \mathbb{P} \rightarrow X$.

BULletin DE LA SOCIÉtÉ MATHÉMATIQUE DE FRANCE 
On a alors le diagramme commutatif de morphismes de topos suivant :

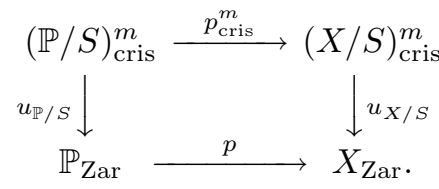

On note

$$
\xi=c_{1, m}\left(\mathscr{O}_{\mathbb{P}}(1)\right) \in H_{m-\text { cris }}^{2}(\mathbb{P}) .
$$

On peut alors voir pour tout $i, \xi^{i}$ comme un morphisme dans $D^{+}\left((X / S)_{\text {cris }}^{m}, \mathrm{Ab}\right)$ :

$$
\xi^{i}: \mathbb{Z}_{X} \longrightarrow \mathbb{R} p_{\text {cris* }}^{m} \mathscr{O}_{\mathbb{P} / S}^{m}[2 i] .
$$

De même, le morphisme $p_{\text {cris }}^{m}$ nous donne un morphisme

$$
\mathscr{O}_{X / S}^{m} \longrightarrow \mathbb{R} p_{\text {cris* }}^{m} \mathscr{O}_{\mathbb{P} / S}^{m} \text {. }
$$

On en déduit alors comme dans le cas classique :

$$
\bigoplus_{i=0}^{r-1} \xi^{i}: \bigoplus_{i=0}^{r-1} \mathscr{O}_{X / S}^{m}[-2 i] \longrightarrow \mathbb{R} p_{\text {cris } *}^{m} \mathscr{O}_{\mathbb{P} / S}^{m} .
$$

Comme nous allons avoir besoin d'une comparaison avec la cohomologie de de Rham, nous nous contenterons de regarder la situation après tensorisation par $\mathbb{Q}$. En effet, contrairement au cas de niveau 0 , le théorème ce comparaison entre la cohomologie $m$-cristalline et la cohomologie de de Rham n'est vrai que modulo torsion [3] (voir cependant la remarque ci-dessous).

Par tensorisation par $\mathbb{Q}$, on déduit de (9) une application

$$
\bigoplus_{i=0}^{r-1} \xi^{i}: \bigoplus_{i=0}^{r-1} \mathscr{O}_{X / S}^{m} \otimes \mathbb{Q}[-2 i] \longrightarrow \mathbb{R} p_{\text {cris* }}^{m} \mathscr{O}_{\mathbb{P} / S}^{m} \otimes \mathbb{Q} .
$$

Proposition 5.11. - Avec les notations précédentes, l'application (10) est un isomorphisme.

Démonstration. - On remarque que, la question étant locale, on peut supposer que $\mathscr{E}=\mathscr{O}_{X}^{r}$ et qu'il existe une immersion fermée de $X$ dans $Y$ un $S$-schéma formel lisse. En notant $\widehat{\mathfrak{P}}$ l'enveloppe à puissance $m$-divisée de cette immersion, on a le diagramme :

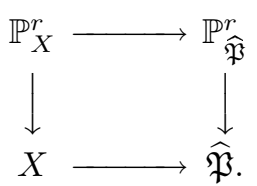

Dans cette situation, la cohomologie $m$-cristalline se calcule comme la cohomologie de de Rham de l'enveloppe à puissances divisées. Le faisceau $\mathscr{O}_{\mathbb{P}_{X}^{r}}(1)$ se relevant en $\mathscr{O}_{\mathbb{P}_{\widehat{\mathfrak{P}}}^{r}}(1)$, on est ramené au même problème pour la cohomologie de de Rham de $\mathbb{P}^{r}$ sur $\widehat{\mathfrak{P}}$. La proposition est connue dans ce cas [20].

TOME $131-2003-\mathrm{N}^{\mathrm{O}} 1$ 
On note

$$
H_{m, \mathbb{Q}}^{i}(X):=H^{i}\left((X / S)_{\mathrm{cris}}^{m}, \mathscr{O}_{X / S} \otimes \mathbb{Q}\right) .
$$

Corollaire 5.12. - Avec les notations ci-dessus, on a pour tout $n$ la décomposition suivante:

$$
H_{m, \mathbb{Q}}^{n}(\mathbb{P})=\bigoplus_{i=0}^{r-1} H_{m, \mathbb{Q}}^{n-2 i}(X) .
$$

On définit les autres classes de Chern comme dans le cas classique. On applique la décomposition du corollaire 5.12 à $\xi^{r}$ et on obtient

$$
\xi^{r}=\sum_{i=1}^{r}(-1)^{i+1} c_{i, m}^{\mathbb{Q}}(\mathscr{E}) \xi^{r-i},
$$

avec $c_{i, m}^{\mathbb{Q}}(\mathscr{E}) \in H_{m, \mathbb{Q}}^{2 i}(X)$. Par définition $c_{i, m}^{\mathbb{Q}}(\mathscr{E})$ est la $i$-ème classe de Chern de $\mathscr{E}\left(\right.$ comme dans la section 4 , on note $c_{0, m}^{\mathbb{Q}}(\mathscr{E})=1$ dans $\left.H_{m, \mathbb{Q}}^{0}(X)\right)$.

Proposition 5.13 (additivité). - Soient $X$ une $k$-variété, $\mathscr{E}$, $\mathscr{E}$ et $\mathscr{E}^{\prime \prime}$ trois faisceaux localement libres de rang $r, r^{\prime}$ et $r^{\prime \prime}$, tels que l'on ait la suite exacte:

$$
0 \rightarrow \mathscr{E}^{\prime} \longrightarrow \mathscr{E} \longrightarrow \mathscr{E}^{\prime \prime} \longrightarrow 0 \text {. }
$$

Pour tout $m$ et tout $i$, on a dans $H_{m, \mathbb{Q}}^{2 i}(X)$

$$
c_{i, m}^{\mathbb{Q}}(\mathscr{E})=\sum_{j=0}^{i} c_{j, m}^{\mathbb{Q}}\left(\mathscr{E}^{\prime}\right) c_{i-j, m}^{\mathbb{Q}}\left(\mathscr{E}^{\prime \prime}\right)
$$

Démonstration. - La fonctorialité de nos constructions permet de les généraliser au cas des variétés simpliciales. On peut donc appliquer la méthode classique [8] qui consiste à se ramener à une situation universelle en considérant le topos classifiant du groupe $\operatorname{GL}\left(r^{\prime}, r^{\prime \prime}\right)$. Ce dernier étant le sous-groupe de $\mathrm{GL}(r)$ consistant en les matrices de la forme

$$
\left(\begin{array}{cc}
\mathrm{GL}\left(r^{\prime}\right) & * \\
0 & \mathrm{GL}\left(r^{\prime \prime}\right)
\end{array}\right)
$$

On pourra trouver une démonstration détaillée dans [30].

Pour ramener l'additivité des classes de Chern à la situation universelle sur B.GL $\left(r^{\prime}, r^{\prime \prime}\right)$, on utilise le corollaire 5.12. À partir de là, la cohomologie du topos classifiant est celle d'une variété simpliciale lisse relevable (il s'agit de produits de $\left.\mathrm{GL}\left(r^{\prime}, r^{\prime \prime}\right)\right)$. On déduit donc notre proposition du résultat similaire en cohomologie de de Rham [14, 2.2].

Proposition 5.14. - Soient $X$ une k-variété et $\mathscr{E}$ un faisceau localement libre sur $X$. Pour tous $m, m^{\prime}$ avec $m \leqslant m^{\prime}$, on a pour tout $i$

$$
\phi_{m^{\prime}, m} c_{i, m^{\prime}}^{\mathbb{Q}}(\mathscr{E})=p^{i\left(m^{\prime}-m\right)} c_{i, m}^{\mathbb{Q}}(\mathscr{E})
$$

BULletin DE LA SOCIÉtÉ MATHÉMATIQUE DE FRANCE 
où $\phi_{m^{\prime}, m}: H^{2 i}\left((X / S)_{\text {cris }}^{m^{\prime}}, \mathscr{O}_{X / S}^{m^{\prime}}\right) \rightarrow H^{2 i}\left((X / S)_{\text {cris }}^{m}, \mathscr{O}_{X / S}^{m}\right)$ est le morphisme induit par $u_{m^{\prime}, m}$.

Démonstration. - Il suffit de le voir pour la première classe de Chern d'un faisceau inversible. Ce dernier cas découle directement de la définition.

Remarque 5.15. - Soient $X$ une $k$-variété et $X^{(m)}$ le pull-back par le $m$ ième itéré du Frobenius de $k$. Pour tout faisceau localement libre $\mathscr{E}$ sur $X$, on notera $\mathscr{E}^{m}$ son pull-back sur $X^{(m)}$. On trouve dans [3] l'isomorphisme

$$
F_{m}^{*}: H_{\text {cris }}^{2 i}\left(X^{(m)}\right) \stackrel{\sim}{\longrightarrow} H_{m \text {-cris }}^{2 i}(X) .
$$

Cela permet, en se ramenant au cas de niveau 0 , de démontrer un analogue de la proposition 5.11 sans avoir besoin de tensoriser par $\mathbb{Q}$. On peut alors définir des classes de Chern $c_{i, m}(\mathscr{E})$ à valeurs dans $H_{m}^{2 i}(X)$. De plus, la classe de Chern $c_{i, m}(\mathscr{E})$ est l'image de la classe $c_{i, 0}(\mathscr{E}(m))$ par le morphisme $F_{m}^{*}$. On déduit alors de l'additivité des classes de Chern cristallines de niveau 0, l'additivité des classes de Chern $m$-cristallines entières.

5.3. Le topos $(\boldsymbol{X} / \boldsymbol{S})_{\text {cris }}^{\bullet}$ - Nous allons construire un topos permettant de calculer la cohomologie rigide de manière cristalline. Ce topos se construit comme le topos associé à un diagramme de topos [SGA4, IV,7] ou [23, VI]. Le cas particulier nous intéressant, à savoir le cas des systèmes inductifs de topos, est repris dans $[30, \mathrm{II}]$.

DÉFInition 5.16. - Avec les notations précédentes, on notera $(X / S)_{\text {cris }}^{\bullet}$ le topos associé au diagramme de topos $\left((X / S)_{\mathrm{cris}}^{m}, i_{m^{\prime}, m}\right)_{m \in \mathbb{N}}$.

Notations 5.17. - On notera souvent un objet de ce topos de la manière suivante :

$$
\mathscr{F}^{\bullet}=\left(\mathscr{F}^{0} \leftarrow \cdots \leftarrow \mathscr{F}^{m} \leftarrow \cdots\right) .
$$

On pose alors

$$
\mathscr{O}_{X / S}^{\bullet}=\left(\mathscr{O}_{X / S}^{0} \leftarrow \cdots \leftarrow \mathscr{O}_{X / S}^{m} \leftarrow \cdots\right),
$$

les flèches de transition étant, pour $m \leqslant m^{\prime}$, les morphismes $u_{m^{\prime}, m}$ définis précédemment. De la même manière, on pose

$$
\mathscr{I}_{X / S}=\left(\mathscr{I}_{X / S}^{0} \leftarrow \cdots \leftarrow \mathscr{I}_{X / S}^{m} \leftarrow \cdots\right) .
$$

On considère alors le topos $(X / S)_{\text {cris }}^{\bullet}$ comme annelé par $\mathscr{O}_{X / S}^{\bullet}$. On a les morphismes de topos annelés suivants :

$$
p_{m}:\left((X / S)_{\mathrm{cris}}^{m}, \mathscr{O}_{X / S}^{m}\right) \longrightarrow\left((X / S)_{\mathrm{cris}}^{\bullet}, \mathscr{O}_{X / S}^{\bullet}\right) .
$$

Le lemme 5.9 nous dit que les morphismes de topos

$$
u_{X / S}^{m}:(X / S)_{\text {cris }}^{m} \longrightarrow X_{\text {Zar }} \quad\left(\text { resp. } i_{X / S}^{m}: X_{\text {Zar }} \longrightarrow(X / S)_{\text {cris }}^{m}\right)
$$

TOME $131-2003-\mathrm{N}^{\mathrm{O}} 1$ 
permettent de construire un morphisme de topos

$$
u_{X / S}^{\bullet}:(X / S)_{\text {cris }}^{\bullet} \rightarrow X_{\text {Zar }}^{\bullet} \quad\left(\text { resp. } i_{X / S}^{\bullet}: X_{\text {Zar }}^{\bullet} \rightarrow(X / S)_{\text {cris }}^{\bullet}\right) .
$$

De même, les foncteurs sections globales $\Gamma\left((X / S)_{\text {cris }}^{m},-\right):(X / S)_{\text {cris }}^{m} \rightarrow$ (Ens) donnent naissance à un foncteur

$$
\Gamma^{\bullet}\left((X / S)_{\text {cris }}^{\bullet},-\right):(X / S)_{\text {cris }}^{\bullet} \longrightarrow(\text { Ens })^{\bullet} .
$$

De plus, l'égalité de foncteurs

$$
\Gamma\left((X / S)_{\mathrm{cris}}^{m},-\right)=\Gamma\left(X_{\mathrm{Zar}},-\right) \circ u_{X / S *}^{m}
$$

implique que

$$
\Gamma^{\bullet}\left((X / S)_{\text {cris }}^{\bullet},-\right)=\Gamma^{\bullet}\left(X_{\text {Zar }}^{\bullet},-\right) \circ u_{X / S *}^{\bullet}
$$

où $\Gamma^{\bullet}\left(X_{\text {Zar }}^{\dot{Z}_{\text {ar }}},-\right)$ est le foncteur de $X_{\text {Zar }}^{\dot{Z}_{\text {ar }}}$ dans (Ens) ${ }^{\bullet}$ défini de la même manière que $\Gamma^{\bullet}\left((X / S)_{\text {cris }}^{\bullet},-\right)$ et o désigne la composition des foncteurs.

REMARQUe 5.18. - On fera attention à ne pas confondre les deux foncteurs

$$
\Gamma^{\bullet}\left((X / S)_{\text {cris }}^{\bullet},-\right) \text { et } \Gamma^{\bullet}\left(X_{\mathrm{Z} \text { ar }}^{\bullet},-\right)
$$

avec les foncteurs sections globales des topos $(X / S)_{\text {cris }}^{\bullet}$ et $X_{\text {Zar }}^{\cdot}$. On a cependant $\Gamma\left((X / S)_{\text {cris }}^{\bullet},-\right)=\lim _{\longleftarrow} \circ \Gamma^{\bullet}\left((X / S)_{\text {cris }}^{\bullet},-\right), \quad \Gamma\left(X_{\text {Zar }}^{\bullet},-\right)=\lim _{\longleftarrow} \circ \Gamma^{\bullet}\left(X_{\text {Zar }}^{\bullet},-\right)$.

Notations 5.19. - Par la suite, le complexe $\mathbb{R} \varliminf_{m}\left(\left(\mathbb{R} u_{X / S_{*}} \mathscr{O}_{X / S}^{\bullet}\right) \otimes \mathbb{Q}\right)$ sera noté $\mathbb{R} \underline{\Gamma}_{\text {crise }}(X)$ et

$$
H_{\text {crisø }}^{*}(X):=H^{*}\left(X_{\dot{\mathrm{Z}}_{\mathrm{ar}}},\left(\mathbb{R} u_{X / S_{*}}^{\bullet} \mathscr{O}_{X / S}^{\bullet}\right) \otimes \mathbb{Q}\right)=H^{*}\left(X_{\mathrm{Zar}}, \mathbb{R} \underline{\Gamma}_{\text {cris }}(X)\right) .
$$

5.4. Construction des classes de Chern $\operatorname{sur}(X / S)_{\text {cris }}^{\bullet^{*}}-$ La construction est similaire à celle des classes de Chern $m$-cristallines.

Avec les notations précédentes, on a la suite exacte dans $(X / S)_{\text {cris }}^{\bullet}$ :

$$
0 \rightarrow 1+\mathscr{I}_{X / S}^{\bullet} \longrightarrow\left(\mathscr{O}_{X / S}^{*}\right)^{\bullet} \longrightarrow i_{*}^{\bullet}\left(\mathscr{O}_{X}^{*}\right) \rightarrow 0 .
$$

Pour le démontrer, il suffit d'utiliser la famille conservative des $p_{m}^{-1}$. On obtient donc un morphisme dans $D^{+}\left((X / S)_{\text {cris }}^{\bullet_{r}}, \mathrm{Ab}\right)$ :

$$
i_{*}^{\bullet}\left(\mathscr{O}_{X}^{*}\right)[1] \longrightarrow\left(1+\mathscr{I}_{X / S}^{\bullet}\right)[2] .
$$

En projetant ce dernier sur le topos zariskien, on obtient dans $D^{+}\left(X_{\text {Zar }}, \mathrm{Ab}\right)$ le morphisme

$$
\left(\mathscr{O}_{X}^{*}\right)^{\bullet}[1] \rightarrow \mathbb{R} u_{X / S *}^{\bullet}\left(1+\mathscr{I}_{X / S}^{\bullet}\right)[2] .
$$

Cependant, les morphismes $\psi_{m}$ définis précédemment ne construisent pas un morphisme sur les systèmes projectifs car ils ne commutent pas aux flèches des systèmes projectifs.

On considère le système projectif

$$
\left(1+\mathscr{I}_{X / S}\right)(1)=\left(1+\mathscr{I}_{X / S}^{0} \stackrel{p}{\longleftarrow} \cdots \stackrel{p}{\longleftarrow} 1+\mathscr{I}_{X / S}^{m} \stackrel{p}{\longleftarrow} \cdots\right)
$$

BULletin DE LA SOCIÉtÉ MATHÉMATIQUE DE FRANCE 
où les flèches de transition sont l'élévation à la puissance $p$ des flèches classiques.

Les morphismes $\psi_{m}$ définissent alors

$$
\psi^{\bullet}:\left(1+\mathscr{I}_{X / S}^{\bullet}\right)(1) \longrightarrow \mathscr{J}_{X / S}^{\bullet}
$$

Après projection sur le topos zariskien, on obtient

$$
\mathbb{R} u_{X / S *}^{\bullet}\left(\left(1+\mathscr{I}_{X / S}^{\bullet}\right)(1)\right)[2] \longrightarrow \mathbb{R} u_{X / S *}^{\bullet}\left(\mathscr{J}_{X / S}^{\bullet}\right)[2]
$$

En composant cette dernière avec la projection du morphisme $\mathscr{J}_{X / S} \rightarrow \mathscr{O}_{X / S}^{\bullet}$, on a

$$
\mathbb{R} u_{X / S *}^{\bullet}\left(\left(1+\mathscr{I}_{X / S}^{\bullet}\right)(1)\right)[2] \longrightarrow \mathbb{R} u_{X / S *}^{\bullet}\left(\mathscr{O}_{X / S}^{\bullet}\right)[2] .
$$

On peut d'autre part construire un morphisme de $\left(1+\mathscr{I}_{X / S}^{\bullet}\right)(1)$ dans $1+$ $\mathscr{I}_{X / S}$ défini par l'élévation à la puissance $p^{m}$ en degré $m$.

On remarque alors que ce morphisme induit après tensorisation par $\mathbb{Q}$ un isomorphisme dans $D^{+}\left(X_{\mathrm{Z}}^{\bullet}, \mathrm{Ab}\right)$ :

$$
\mathbb{R} u_{X / S *}^{\bullet}\left(\left(1+\mathscr{I}_{X / S}^{\bullet}\right)(1)\right) \otimes \mathbb{Q} \stackrel{\sim}{\longrightarrow} \mathbb{R} u_{X / S *}^{\bullet}\left(1+\mathscr{I}_{X / S}^{\bullet}\right) \otimes \mathbb{Q} .
$$

En composant (11), l'inverse de (13) et (12), on obtient, après passage à la limite

$$
c_{1, \bullet}:\left(\mathscr{O}_{X}^{*}\right)[1] \longrightarrow \mathbb{R} \underline{\Gamma}_{\text {cris }}(X)[2] .
$$

Par suite en passant à la cohomologie, on a

$$
c_{1, \bullet}: H^{1}\left(X, \mathscr{O}_{X}^{*}\right) \longrightarrow H_{\text {cris }}^{2}(X) \text {. }
$$

Comme dans le cas $m$-cristallin, la première classe de Chern permet de calculer la cohomologie des fibrés projectifs. On peut donc construire classiquement les autres classes de Chern.

Remarque 5.20. - Comme dans la section 4, on note $c_{0}(\mathscr{E})=1$ dans $H_{\text {cris }}^{0}(X)$. De plus, si $\mathscr{E}$ est un faisceau inversible, on retrouve la première classe de Chern construite auparavant.

On démontre alors, comme pour la cohomologie $m$-cristallines, que l'on a :

Proposition 5.21. - Les classes de Chern $c_{i, \bullet}$ vérifient les propriétés d'additivité et de fonctorialité.

5.5. Interprétation cristalline de la cohomologie rigide. - Les définitions précédentes permettent d'énoncer l'interprétation cristalline de la cohomologie rigide.

THÉORÈme 5.22 (Berthelot). — Soit X une k-variété propre. En reprenant les notations précédentes, on a

$$
\mathbb{R} \underline{\Gamma}_{\text {rig }}(X / K) \stackrel{\sim}{\longrightarrow} \mathbb{R} \underline{\Gamma}_{\text {cris }}(X) .
$$

TOME $131-2003-\mathrm{N}^{\mathrm{O}} 1$ 
Démonstration. - Remarquons juste que la démonstration de ce théorème utilise un calcul de la cohomologie $m$-cristalline à l'aide de la cohomologie de de Rham qui ne se fait qu'à torsion près, il est donc nécessaire de tensoriser par $\mathbb{Q}$. La démonstration se trouve dans [3].

En passant à la cohomologie, on obtient

$$
H_{\text {rig }}^{i}(X / K) \stackrel{\sim}{\longrightarrow} H_{\text {cris }}^{i}(X) .
$$

5.6. Comparaison avec les classes de Chern rigides : cas des variétés propres. - Nous allons voir que les classes de Chern que nous venons de construire coïncident avec les classes de Chern rigides définies à la section 4 . On se donne $X$, une $k$-variété propre, ainsi qu'un plongement $X \hookrightarrow \mathscr{Y}$ dans un schéma formel sur $S=\operatorname{Spf}(C)$ lisse au voisinage de $X$.

THÉORÈme 5.23. - On a le diagramme commutatif suivant

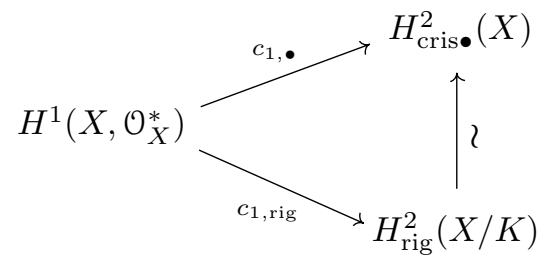

où $c_{1, \bullet}$ est le morphisme (15) et la flèche verticale se déduit de celle de 5.22.

Corollaire 5.24. - Soit $X$ une variété propre. Les classes de Chern rigides sur $X$ vérifient la propriété d'additivité.

Démonstration du théorème 5.23. — Nous allons, comme dans [9], réinterpréter la construction de nos classes de Chern cristallines à l'aide de faisceaux zariskiens. Nous utiliserons alors des résolutions de Čech afin de les comparer avec la définition de la section 4 .

Dans $D^{+}\left(X_{\text {Zar }}\right)$ nous noterons avec un $\mathbb{Q}$ en indice les complexes obtenus après tensorisation par $\mathbb{Q}$.

On notera $Y_{n}$ la réduction modulo $p^{n+1}$ de $\mathscr{Y}$, pour tout $m$ et tout $n$, on note alors $\mathfrak{P}_{n}^{m}$ l'enveloppe à puissance $m$-divisée de l'immersion de $X$ dans $Y_{n}$, $\widehat{\mathfrak{P}}^{m}$ sa complétion $p$-adique et $\widehat{\mathfrak{P}}^{\bullet}$ le système projectif associé.

On regarde le complexe de de Rham et le complexe de de Rham multiplicatif

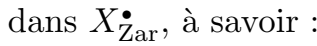

$$
\Omega_{\widehat{\mathfrak{P}} \bullet / S}^{\star}=\mathscr{O}_{\widehat{\mathfrak{P}}} \stackrel{\mathrm{d}}{\longrightarrow} \Omega_{\widehat{\mathfrak{P}} \bullet / S}^{1} \longrightarrow \Omega_{\widehat{\mathfrak{P}} / S}^{2} \rightarrow \cdots
$$

et

$$
\Omega_{\widehat{\mathfrak{P}} \bullet / S}^{\times}=\mathscr{O}_{\widehat{\mathfrak{P}}}^{*} \stackrel{\mathrm{d} \log }{\longrightarrow} \Omega_{\widehat{\mathfrak{P}} \bullet / S}^{1} \longrightarrow \Omega_{\widehat{\mathfrak{P}} / S}^{2} \rightarrow \cdots
$$

BULlETIN DE LA SOCIÉtÉ MATHÉMATIQUE DE FRANCE 
et on note $\mathscr{K}_{\hat{\mathfrak{P}} \bullet / S}^{\times}\left(\right.$resp. $\left.\mathscr{I}_{\widehat{\mathfrak{P}} \bullet / S}^{\star}\right)$ le noyau du morphisme $\Omega_{\widehat{\mathfrak{P}} \bullet / S}^{\times} \rightarrow\left(\mathscr{O}_{X}^{*}\right)^{\bullet}$ (resp. $\left.\Omega_{\widehat{\mathfrak{F}} \bullet / S}^{\star} \rightarrow\left(\mathscr{O}_{X}\right)^{\bullet}\right)$. On rappelle que $\mathscr{I}^{m}$ est l'idéal de $X$ dans $\widehat{\mathfrak{P}}^{m}$.

De plus, comme précédemment, on note

$$
\begin{aligned}
(1+\mathscr{I} \bullet)(1) & =\left(1+\mathscr{I}^{0} \stackrel{p}{\longleftarrow} 1+\mathscr{I}^{1} \stackrel{p}{\longleftarrow} \ldots \stackrel{p}{\longleftarrow} 1+\mathscr{I}^{m} \stackrel{p}{\longleftarrow} \cdots\right), \\
\Omega_{\mathfrak{\mathfrak { P }}^{i} \bullet / S}^{i}(1) & =\left(\Omega_{\mathfrak{\mathfrak { P }}^{0} / S}^{i} \stackrel{p}{\longleftarrow} \Omega_{\mathfrak{\mathfrak { P }}^{1} / S}^{i} \stackrel{p}{\longleftarrow} \ldots \stackrel{p}{\longleftarrow} \Omega_{\widehat{\mathfrak{P}}^{m} / S}^{i} \stackrel{p}{\longleftarrow} \cdots\right) .
\end{aligned}
$$

Enfin on pose

$$
\mathscr{K}_{\widehat{\mathfrak{P}} \bullet / S}^{\times}(1)=(1+\mathscr{I} \bullet)(1) \stackrel{\mathrm{d} \log }{\longrightarrow} \Omega_{\widehat{\mathfrak{P}} \bullet / S}^{1}(1) \longrightarrow \Omega_{\widehat{\mathfrak{P}} \bullet / S}^{2}(1) \rightarrow \cdots .
$$

On a alors un morphisme

$$
\left(\mathscr{K}_{\hat{\mathfrak{P}} \bullet / S}^{\times}(1)\right)_{\mathbb{Q}} \longrightarrow\left(\Omega_{\hat{\mathfrak{P}} \bullet / S}^{\star}\right)_{\mathbb{Q}}
$$

défini par les applications

$$
\begin{aligned}
p^{m} \log & :\left(1+\mathscr{I}^{m}\right) \longrightarrow \mathscr{O}_{\widehat{\mathfrak{P}}^{m}}, \\
\times p^{m} & : \Omega_{\widehat{\mathfrak{P}}^{m} / S}^{i} \longrightarrow \Omega_{\mathfrak{\mathfrak { P }}^{m} / S}^{i} \quad \text { pour } i \geqslant 1 .
\end{aligned}
$$

Avec ces notations, on a le diagramme commutatif suivant dans $D^{+}\left(X_{\text {Zar }}\right)$ :

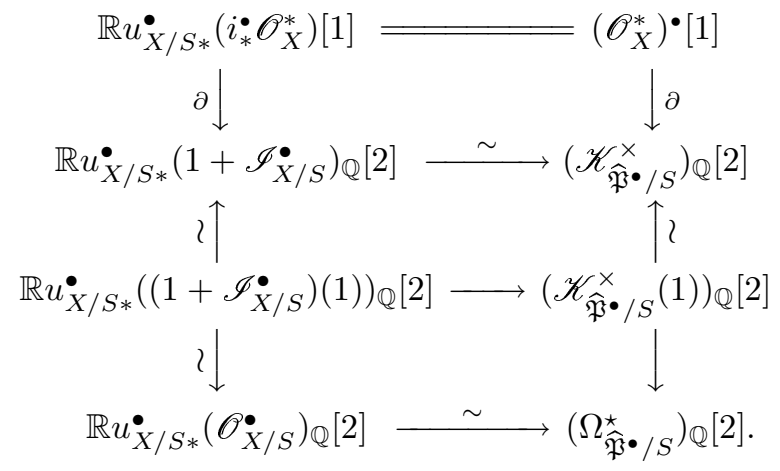

La première classe de Chern étant, par définition, l'application induite sur la cohomologie par la première ligne du diagramme, elle peut être calculée par la deuxième. Nous allons maintenant en faire un calcul explicite à l'aide de cocycles de Cech.

On sait que pour tout $\sigma \in H^{1}\left(X, \mathscr{O}_{X}^{*}\right)$, il existe un recouvrement $\mathfrak{U}$ de $\mathscr{Y}$ tel que $\sigma$ soit l'image d'un élément (encore noté $\sigma$ ) de $H^{1}\left(\mathfrak{U}_{X}, \mathscr{O}_{X}^{*}\right)$.

Notations 5.25. - On reprend les notations de la section 3. On notera :

- $\mathscr{I}_{i}^{m}$ l'idéal de $X_{i}$ dans $\widehat{\mathfrak{P}}_{i}^{m}$,

- $I_{i}^{m}=\Gamma\left(\widehat{\mathfrak{P}}_{i}^{m}, \mathscr{I}_{i}^{m}\right)$ et

- $\widehat{\mathfrak{P}}_{i}^{m}=\operatorname{Spf}\left(\widehat{P}_{i}^{m}\right)$.

TOME $131-2003-\mathrm{N}^{\mathrm{O}} 1$ 
En notant $P_{n, i}^{m}$ l'enveloppe à puissances divisées de niveau $m$ de

$$
\left(\mathscr{A}_{i} / p^{n+1} \mathscr{A}_{i}, I_{i} / p^{n+1} I_{i}\right)
$$

où $I_{i}$ est l'idéal de $A_{i}$ dans $\mathscr{A}_{i}$, on a

$$
\widehat{P}_{i}^{m}={\underset{n}{n}}_{\lim _{n, i}} P^{m}
$$

On a alors des morphismes

$$
\pi_{i}^{m}: \widehat{P}_{i}^{m} \longrightarrow A_{i}
$$

Pour tout $m^{\prime}>m$, on notera $\varepsilon_{i}^{m, m^{\prime}}$ la flèche entre $\widehat{P}_{i}^{m^{\prime}}$ et $\widehat{P}_{i}^{m}$. Dans le cas particulier où $m^{\prime}=m+1$, nous la noterons simplement $\varepsilon_{i}^{m}$.

Avec ces notations, $\sigma$ est donc représenté par un cocycle $u_{i j} \in C^{1}\left(\mathfrak{U}_{X}, \mathscr{O}_{X}^{*}\right)$. On regarde donc le cocycle $u_{i j}^{m} \in C^{1}\left(\mathfrak{U},\left(\mathscr{O}_{X}^{*}\right)^{\bullet}\right)$ défini par $u_{i j}^{m}=u_{i j}$ pour tout $m$. Ce cocycle définit une application dans $D^{+}\left(X_{\text {Zar }}\right): \mathbb{Z} \rightarrow\left(\mathscr{O}_{X}^{*}\right)^{\bullet}[1]$. Nous allons montrer que l'application de $D^{+}\left(X_{\mathrm{Zar}}\right)$,

$$
\mathbb{Z} \longrightarrow\left(\Omega_{\hat{\mathfrak{P}} \bullet / S}^{\star}\right)_{\mathbb{Q}}[2]
$$

obtenue comme composition de cette application avec la deuxième ligne du

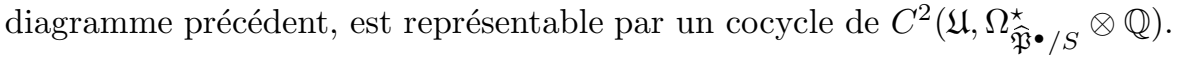

DÉfinition 5.26. - Avec les notations précédentes, pour tout $x \in A_{i}$, on appelle relèvement compatible de $x$ une famille $\left(\widetilde{x}^{m}\right) \in \Pi_{m \in \mathbb{N}} \widehat{P}_{i}^{m}$ telle que :

- on ait $\pi_{i}^{m}\left(\widetilde{x}^{m}\right)=x$ pour tout $m$;

- on ait $\varepsilon_{i}^{m, m^{\prime}}\left(\widetilde{x}^{m^{\prime}}\right)=\widetilde{x}^{m}$ pour tout $m^{\prime}>m$.

Le diagramme commutatif

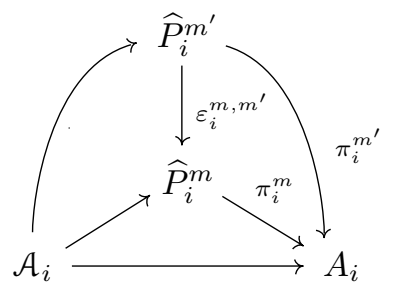

montre que pour construire un relèvement compatible, il suffit de relever $x$ dans $\mathscr{A}_{i}$ et d'utiliser les morphismes $\mathscr{A}_{i} \rightarrow \widehat{P}_{i}^{m}$. En particulier, il existe toujours des relèvements compatibles.

On appellera système compatible un élément vérifiant la deuxième condition.

Soit $\left(\widetilde{u}_{i j}^{m}\right) \in \Pi_{m \in \mathbb{N}} \widehat{P}_{i j}^{m}$ un relèvement compatible de $u_{i j}=u_{i j}^{m}$.

Lemme 5.27. - Avec les notations précédentes, $\widetilde{u}_{i j}^{m}$ est inversible dans $\widehat{P}_{i j}^{m}$.

BULLETIN DE LA SOCIÉTÉ MATHÉMATIQUe DE FRANCE 
Démonstration. - On relève aussi $\left(u_{i j}\right)^{-1}$ de manière compatible en $\widetilde{v}_{i j}^{m}$. On a alors

$$
\widetilde{u}_{i j}^{m} \cdot \widetilde{v}_{i j}^{m}=1+x_{i j}^{m} \in 1+I_{i j}^{m} .
$$

De plus, $I_{i j}^{m}$ étant un $m$-PD-idéal, pour tout $x \in I_{i j}^{m}$ et tout $k \in \mathbb{N}$, on a

$$
x^{k}=q_{k} !\left(x^{p^{m}}\right)^{\left[q_{k}\right]} x^{r_{k}}
$$

où $k=p^{m} q_{k}+r_{k}, 0 \leqslant r_{k}<p^{m}$ et [·] désigne les puissances divisées. À partir de là, on voit que $x^{k}$ tend $p$-adiquement vers 0 quand $k$ tend vers l'infini. Comme $\widehat{P}_{i j}^{m}$ est complet, la série

$$
\sum_{k=0}^{\infty}\left(-x_{i j}^{m}\right)^{k}
$$

converge vers $\left(1+x_{i j}^{m}\right)^{-1}$. L'inverse de $\widetilde{u}_{i j}^{m}$ est donc

$$
\widetilde{v}_{i j}^{m} \cdot \sum_{k=0}^{\infty}\left(-x_{i j}^{m}\right)^{k}
$$

Remarque 5.28. - Ce lemme est l'analogue $m$-cristallin du lemme 3.4.

De plus, il est clair d'après la définition que $\widetilde{x}_{i j}^{m}$ et donc $\left(\widetilde{u}_{i j}^{m}\right)^{-1}$ sont des systèmes compatibles. À partir de là, on considère

$$
\frac{\mathrm{d} \widetilde{u}_{i j}^{m}}{\widetilde{u}_{i j}^{m}} \in C^{1}\left(\mathfrak{U}, \Omega_{\widehat{\mathfrak{P}}^{m} / S}^{1}\right) \quad \text { et } \quad h_{i j k}^{m}=\widetilde{u}_{i j}^{m} \cdot\left(\widetilde{u}_{i k}^{m}\right)^{-1} \cdot \widetilde{u}_{j k}^{m} \in C^{2}\left(\mathfrak{U}, 1+\mathscr{I}^{m}\right) .
$$

Ils définissent un élément de $C^{2}\left(\mathfrak{U}_{,} \mathscr{K}_{\widehat{\hat{\mathfrak{P}}} \bullet / S}^{\times}\right)$. Des calculs similaires à ceux de la section 3 montrent que si on modifie les relèvements choisis ou le choix du cocycle, on modifie cette classe d'un cobord. On obtient donc bien une application dans la catégorie dérivée :

$$
\mathbb{Z} \longrightarrow \mathscr{K}_{\hat{\mathfrak{P}} \bullet / S}^{\times}[2] .
$$

On inverse alors, après tensorisation par $\mathbb{Q}$, le morphisme $\mathscr{K}_{\hat{\mathfrak{P}} \bullet / S}^{\times} \otimes \mathbb{Q} \rightarrow$ $\mathscr{K}_{\widehat{\mathfrak{N}} \bullet / S}^{\times}(1) \otimes \mathbb{Q}$ en considérant le morphisme qui est défini en degré $m$ par la division par $p^{m}$. On obtient donc le cocycle :

$$
\left(\frac{\mathrm{d} \widetilde{u}_{i j}^{m}}{\widetilde{u}_{i j}^{m}} \otimes \frac{1}{p^{m}}, \widetilde{u}_{i j}^{m} \cdot\left(\widetilde{u}_{i k}^{m}\right)^{-1} \cdot \widetilde{u}_{j k}^{m} \otimes \frac{1}{p^{m}}\right) \in C^{2}\left(\mathfrak{U}, \mathscr{K}_{\hat{\mathfrak{P}}_{\bullet} / S}^{\times}(1) \otimes \mathbb{Q}\right) .
$$

On conclut alors notre calcul en utilisant l'application égale en degré $m$ à la multiplication par $p^{m}$ sur la première composante et $p^{m} \log$ sur la deuxième. Ce qui nous donne

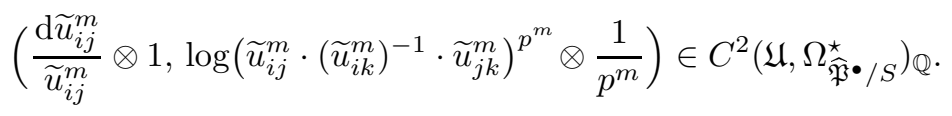

TOME $131-2003-\mathrm{N}^{\mathrm{O}} 1$ 
Ce cocycle correspond, par le morphisme du théorème 5.22 , à celui qui a été construit à la section 3 .

Avant de passer aux variétés ouvertes, donnons deux autres corollaires du théorème 5.23 .

Corollaire 5.29. - Soit X une k-variété propre; on a le diagramme commutatif suivant

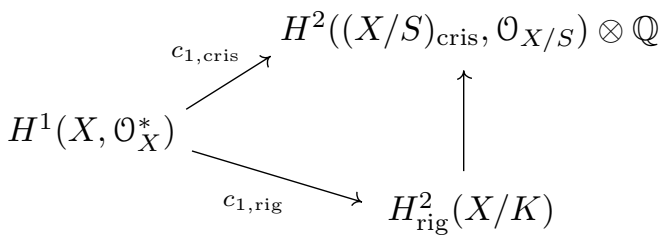

où la flèche $c_{1, \mathrm{cris}}$ est la première classe de Chern cristalline de [8] et la flèche verticale est celle qui est donnée par

$$
H_{\text {rig }}^{2}(X / K) \longrightarrow H_{\text {cris }}^{2}(X) \longrightarrow H^{2}\left((X / S)_{\text {cris }}, \mathscr{O}_{X / S}\right) \otimes \mathbb{Q}
$$

Démonstration. — Grâce à la proposition, il suffit de comparer $c_{1}^{\bullet}$ avec $c_{1, \text { cris }}$. Or cette dernière s'obtient à partir de la première en appliquant le foncteur $p_{0}^{*}$.

Soit $X$ une variété propre. Il existe un théorème de comparaison entre la cohomologie rigide de $X$ et la cohomologie de son topos convergent $[29,0.6 .7]$. Les classes de Chern rigides sont compatibles à cet isomorphisme.

Corollaire 5.30. - On a

$$
c_{i, \mathrm{rig}}(\mathscr{E})=c_{i, \operatorname{conv}}(\mathscr{E})
$$

via l'isomorphisme

$$
H_{\text {rig }}^{2 i}(X / K) \stackrel{\sim}{\longrightarrow} H^{2 i}\left((X / S)_{\text {conv }}, \mathscr{K}_{X / S}\right)
$$

où $c_{i, \mathrm{conv}}$ sont les classes de Chern définies dans $[28, \mathrm{~A}]$.

Démonstration. - On plonge $X$ dans $\mathscr{Y}$ un $\mathscr{V}$-schéma formel. On regarde alors $T=\left(X, \mathscr{Y}, \operatorname{Id}_{X}\right)$ qui est un enlargement [29, 0.1.1]. En utilisant [4, 2.3.4], on voit que l'on a les isomorphismes

$$
\mathbb{R} \lim _{m}\left(\mathbb{R} u_{X / S *}^{\bullet}\left(\mathscr{O}_{X / S}^{\bullet}\right) \otimes \mathbb{Q}\right) \stackrel{\sim}{\longrightarrow} \mathbb{R s p}_{*} \Omega_{] X[}^{\star} \stackrel{\sim}{\longrightarrow}\left(\mathscr{K}_{X / S}\right)_{T}
$$

Dès lors la construction du $c_{1, \text { conv }}$ de $[28, \mathrm{~A}]$ est la même que celle du $c_{1, \bullet}$ ci-dessus. Les décompositions de $H_{\text {rig }}^{*}\left(\mathbb{P}_{X} / K\right)$ comme $H_{\text {rig }}^{*}(X / K)$-module et $H^{*}\left(\left(\mathbb{P}_{X} / S\right)_{\text {conv }}, \mathscr{K}_{\mathbb{P}_{X} / S}\right)$ comme $H^{*}\left((X / S)_{\text {conv }}, \mathscr{K}_{X / S}\right)$-module étant compatibles, notre comparaison se prolonge aux $c_{i}$. 
5.7. Comparaison avec les classes de Chern rigides : cas des variétés ouvertes. - Nous allons montrer que l'additivité des classes de Chern sur une variété ouverte se ramène au cas des variétés propres. Nous aurons besoin pour cela d'une variante de la proposition 3.21.

Lors de la démonstration du théorème, nous utiliserons le théorème de platification par éclatement de Raynaud-Gruson [33].

Nous allons avoir besoin de terminologie. Soient $X$ une $k$-variété, $Y$ une sous-variété fermée et $\mathscr{F}$ un $\mathscr{O}_{X}$-module. Si on note $\pi: X^{\prime} \rightarrow X$ l'éclaté de $Y$ dans $X$, on appellera transformé strict de $\mathscr{F}$, et on notera $T S(\mathscr{F})$, le quotient de $\pi^{*}(\mathscr{F})$ par le sous-faisceau engendré par les sections à support dans $\pi^{-1}(Y)$.

LEMme 5.31. - Avec les notations précédentes, le transformé strict d'un faisceau cohérent (resp. localement libre) est cohérent (resp. localement libre).

Démonstration. — Soit $\mathscr{F}$ un faisceau cohérent (resp. localement libre). Le faisceau $\mathscr{G}:=\pi^{*}(\mathscr{F})$ est cohérent (resp. localement libre). On note $\mathscr{H}_{\pi^{-1}(Y)}^{0}(\mathscr{G})$ le sous-faisceau de $\mathscr{G}$ engendré par les sections à support dans $\pi^{-1}(Y)$. Si $\mathscr{G}$ est localement libre, on a $\mathscr{H}_{\pi^{-1}(Y)}^{0}(\mathscr{G})=0$ et donc

$$
T S(\mathscr{F})=\mathscr{G} / \mathscr{H}_{\pi^{-1}(Y)}^{0}(\mathscr{G})
$$

est localement libre. Si $\mathscr{G}$ est cohérent, $\mathscr{H}_{\pi^{-1}(Y)}^{0}(\mathscr{G})$ est aussi cohérent. Dès lors $T S(\mathscr{F})$ est cohérent.

Passons à l'énoncé du théorème :

THÉORÈME 5.32. - Soient $X$ une k-variété,

$$
0 \rightarrow \mathscr{E}^{\prime} \longrightarrow \mathscr{E} \longrightarrow \mathscr{E}^{\prime \prime} \rightarrow 0
$$

une suite exacte de faisceaux localement libres sur $X$. Il existe $j: X \hookrightarrow \bar{X}$ une compactification de $X$ et une suite exacte de faisceaux localement libres

$$
0 \rightarrow \overline{\mathscr{E}^{\prime}} \longrightarrow \overline{\mathscr{E}} \longrightarrow \overline{\mathscr{E}}^{\prime \prime} \rightarrow 0
$$

sur $\bar{X}$, telles que la restriction à $X$ de cette dernière soit la suite exacte (16).

Démonstration. - On choisit une compactification $\left(\bar{X}_{1}, j_{1}\right)$ de $X$. Il existe alors un sous-faisceau de $j_{1 *}(\mathscr{E})$ qui soit cohérent et dont la restriction à $X$ soit $\mathscr{E}$. Nommons un tel faisceau $\overline{\mathscr{E}}_{1}$. On regarde alors la flèche composée

$$
\varphi: \overline{\mathscr{E}}_{1} \longrightarrow j_{1 *}(\mathscr{E}) \longrightarrow j_{1 *}\left(\mathscr{E}^{\prime \prime}\right) \text {. }
$$

On pose $\overline{\mathscr{E}}_{1}^{\prime}:=\operatorname{Ker} \varphi, \overline{\mathscr{E}}_{1}^{\prime \prime}:=\overline{\mathscr{E}}_{1} / \overline{\mathscr{E}}_{1}{ }^{\prime}$. On obtient donc une suite exacte

$$
0 \rightarrow \overline{\mathscr{E}}_{1}^{\prime} \longrightarrow \overline{\mathscr{E}}_{1} \longrightarrow \overline{\mathscr{E}}_{1}^{\prime \prime} \rightarrow 0
$$

où $\overline{\mathscr{E}}_{1}^{\prime}, \overline{\mathscr{E}}_{1}$ et $\overline{\mathscr{E}}_{1}{ }^{\prime \prime}$ sont trois faisceaux cohérents. De plus la restriction de la suite exacte (17) à $X$ est la suite exacte (16).

TOME $131-2003-\mathrm{N}^{\mathrm{O}} 1$ 
On sait alors par le théorème de platification par éclatement de RaynaudGruson [33, 5.2.2] qu'il existe un éclatement $p_{1}: \bar{X}_{2} \rightarrow \bar{X}_{1}$ en dehors de $X$ tel que $\overline{\mathscr{E}}_{2}=T S\left(\overline{\mathscr{E}}_{1}\right)$ soit plat. Étant de plus cohérent, $\overline{\mathscr{E}}_{2}$ est localement libre. En posant

$$
\overline{\mathscr{E}}_{2}{ }^{\prime \prime}:=T S\left(\overline{\mathscr{E}}_{1}^{\prime \prime}\right), \quad \overline{\mathscr{E}}_{2}{ }^{\prime}:=\operatorname{Ker}\left(\overline{\mathscr{E}}_{2} \rightarrow \overline{\mathscr{E}}_{2}{ }^{\prime \prime}\right)
$$

on a une suite exacte

$$
0 \rightarrow \overline{\mathscr{E}}_{2}{ }^{\prime} \longrightarrow \overline{\mathscr{E}}_{2} \longrightarrow \overline{\mathscr{E}}_{2}{ }^{\prime \prime} \rightarrow 0
$$

De plus $\overline{\mathscr{E}}_{2}^{\prime}$ et $\overline{\mathscr{E}}_{2}^{\prime \prime}$ sont cohérents, $\overline{\mathscr{E}}_{2}$ est localement libre et la restriction de la suite exacte (18) à $X$ est encore la suite exacte (16). On considère alors un éclatement $p_{2}: \bar{X} \rightarrow \bar{X}_{2}$ en dehors de $X$ (vu comme ouvert de $\bar{X}_{2}$ ) tel que $\overline{\mathscr{E}}^{\prime \prime}=T S\left(\overline{\mathscr{E}}_{2}{ }^{\prime \prime}\right)$ soit localement libre. On procède comme précédemment en posant

$$
\overline{\mathscr{E}}=p_{2}^{*}\left(\overline{\mathscr{E}}_{2}\right), \quad \overline{\mathscr{E}}^{\prime}:=\operatorname{Ker}\left(\overline{\mathscr{E}} \rightarrow \overline{\mathscr{E}}^{\prime \prime}\right)
$$

On obtient alors une suite exacte

$$
0 \rightarrow \overline{\mathscr{E}}^{\prime} \longrightarrow \overline{\mathscr{E}} \longrightarrow \overline{\mathscr{E}}^{\prime \prime} \rightarrow 0
$$

où les deux faisceaux de droite sont localement libres par construction (le transformé strict d'un faisceau localement libre est localement libre) et celui de gauche comme noyau d'une application surjective entre faisceaux localement libres. La restriction à $X$ de cette suite exacte est la suite exacte (16).

Corollaire 5.33. - Les classes de Chern rigides sont additives.

Démonstration. - Soient $X$ une variété et une suite exacte de faisceaux localement libres

$$
0 \rightarrow \mathscr{E}^{\prime} \longrightarrow \mathscr{E} \longrightarrow \mathscr{E}^{\prime \prime} \rightarrow 0
$$

On sait — grâce au théorème 5.32 - qu'il existe une compactification de $X$, $j: X \hookrightarrow \bar{X}$ et une suite exacte de faisceaux localement libres sur $\bar{X}$

$$
0 \rightarrow \overline{\mathscr{E} \prime} \longrightarrow \overline{\mathscr{E}} \longrightarrow \overline{\mathscr{E}}^{\prime \prime} \rightarrow 0
$$

telles que la restriction à $X$ de $\overline{\mathscr{E}}$ (resp. $\left.\overline{\mathscr{E}}^{\prime}, \overline{\mathscr{E}}^{\prime \prime}\right)$ soit $\mathscr{E}$ (resp. $\left.\mathscr{E}^{\prime}, \mathscr{E}^{\prime \prime}\right)$. Dès lors, on a pour tout $i$

$$
c_{i}(\mathscr{E})=j^{*} c_{i}(\overline{\mathscr{E}})=j^{*}\left(\sum_{j=0}^{i} c_{i}\left(\overline{\mathscr{E}}^{\prime}\right) c_{i-j}(\overline{\mathscr{E}} \prime \prime)\right)=\sum_{j=0}^{i} c_{i}\left(\mathscr{E}^{\prime}\right) c_{i-j}\left(\mathscr{E}^{\prime \prime}\right)
$$

BULletin DE LA SOCIÉtÉ MATHÉMATIQUE DE FRANCE 
5.8. Action du Frobenius. - Soit $X$ une $k$-variété. On note $F$ le frobenius absolu sur $X$. Il induit sur les groupes de cohomologie rigide un frobenius

$$
\Phi: H_{\text {rig }}^{i}(X / K) \longrightarrow H_{\text {rig }}^{i}(X / K) \text {. }
$$

On a alors :

Proposition 5.34. - Soit $\mathscr{E}$ un faisceau localement libre de rang $r$ sur $X$, pour tout $i \leqslant r$, on a

$$
\Phi\left(c_{i}(\mathscr{E})\right)=p^{i} c_{i}(\mathscr{E})
$$

Démonstration. - Par la fonctorialité des classes de Chern, notre proposition se ramène à

$$
c_{i}\left(F^{*} \mathscr{E}\right)=p^{i} c_{i}(\mathscr{E})
$$

De plus, de par la définition des classes de Chern, il suffit de regarder le cas du $c_{1}$ d'un faisceau inversible. Or dans ce cas, on a $F^{*} \mathscr{E} \stackrel{\sim}{\rightarrow} \mathscr{E} \otimes p$. Notre proposition découle alors de la multiplicativité des classes de Chern.

\section{Comparaison avec le morphisme de Gysin - Classes de cycles}

Nous allons commencer par construire les classes de cycles. Nous les comparerons ensuite avec la classe d'un pseudo-diviseur afin de montrer qu'elles sont compatibles à l'équivalence rationnelle.

6.1. Définitions et propriétés des classes de cycles. — Par linéarité, on définit le morphisme classe de cycle:

$$
\begin{aligned}
\gamma: Z_{\bullet}(X) & \longrightarrow H_{\bullet}^{\mathrm{rig}}(X), \\
\sum n_{i}\left[T_{i}\right] & \longmapsto \sum n_{i} \alpha_{i *} \eta_{T_{i}},
\end{aligned}
$$

où $\alpha_{i}$ est le morphisme $T_{i} \hookrightarrow X$ et $\alpha_{i *}$ est le morphisme de fonctorialité covariante de l'homologie rigide.

Soit $x$ un $k$-cycle de $X$, on voit que la classe de $x$ se définit dans $H_{2 k}^{\text {rig }}(|x|)$. On notera alors $\gamma^{\prime}(x)$ cette classe.

La classe fondamentale étant fonctorielle par rapport aux immersions ouvertes, on a :

Proposition 6.1. - Soient $j: U \hookrightarrow X$ une immersion ouverte et $x \in A .(X)$. On a

$$
\gamma_{U} j^{*}(x)=j^{*} \gamma_{X}(x)
$$

Nous allons démontrer que les classes de cycles sont fonctorielles et nous regarderons l'action du frobenius. Pour cela, nous allons devoir nous ramener au cas d'un diviseur lisse dans une variété lisse. Nous utiliserons les lemmes suivant [EGAIV, 4.6.6] et [EGAIV, 17.15.12].

TOME $131-2003-\mathrm{N}^{\mathrm{O}} 1$ 
LEMme 6.2. - Soit $X$ une k-variété, il existe une extension finie radicielle $k^{\prime} / k$ tel que le sous-schéma réduit de $X \times_{k} k^{\prime}$ soit géométriquement réduit.

LEMME 6.3. - Soient $X$ une k-variété géométriquement réduite, il existe un ouvert partout dense $U$ qui soit lisse sur $k$

Proposition 6.4. - Soient $X$ et $X^{\prime}$ deux k-variétés. Si $f: X \rightarrow X^{\prime}$ est un morphisme propre et $x \in Z$. $(X)$, on a

$$
\gamma\left(f_{*}(x)\right)=f_{*}(\gamma(x)) .
$$

Démonstration. - Tout étant linéaire, on se ramène au cas d'un sous-schéma fermé intègre $Z$. On note $Z^{\prime}=f(Z), \operatorname{dim} Z=d, \operatorname{dim} X=n$ et $\operatorname{dim} X^{\prime}=n^{\prime}$. On différencie deux cas.

- Si $\operatorname{dim} Z^{\prime}<d$, par définition $f_{*}(Z)=0$. On est donc ramené à montrer que $f_{*}(\gamma(Z))=0$. Le morphisme $f_{\mid Z}: Z \rightarrow Z^{\prime}$ est propre, on a donc le diagramme commutatif suivant :

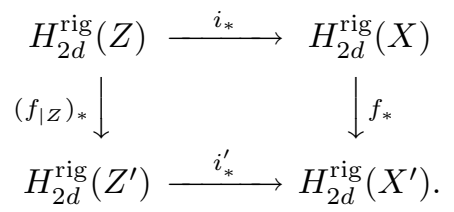

Or on sait que, par définition, $\gamma(Z)=i_{*} \eta_{Z}$. Donc on a

$$
f_{*}(\gamma(Z))=i_{*}^{\prime}\left(\left(f_{\mid Z}\right)_{*}\left(\eta_{Z}\right)\right) \text {. }
$$

Or $H_{2 d}^{\text {rig }}\left(Z^{\prime}\right)=0$. On a donc bien

$$
f_{*}(\gamma(Z))=0
$$

- Si $\operatorname{dim} Z^{\prime}=d$. On peut supposer pour commencer que $X=Z$. On est donc ramené à montrer que

$$
f_{*} \eta_{X}=\eta_{f_{*} X} .
$$

De plus, il suffit grâce à la proposition 2.6 de démontrer cette égalité après une extension finie des scalaires. On peut donc supposer grâce au lemme 6.2 que $X$ est géométriquement réduite. En utilisant le théorème de pureté cohomologique en cohomologie rigide $[7,5.7]$ et le lemme 6.3 , on peut supposer de plus que $f$ est un morphisme fini entre schémas affines et lisses. Comme $X^{\prime}$ est intègre, on sait qu'il existe un ouvert dense $U^{\prime}$ de $X^{\prime}$ au dessus duquel $f$ est plat. On note $U=f^{-1}\left(U^{\prime}\right)$. Se restreindre à $U$ revient à ôter de $X$ et $X^{\prime}$ des sous-schémas fermés de codimension supérieure ou égale à 1 . On peut donc supposer que $f$ est fini et plat de degré $\delta=\left[k(X): k\left(X^{\prime}\right)\right]$. Pour conclure, on utilise le lemme suivant dû à Berthelot qui est une généralisation de $[6,1.4$ et 2.3] :

BULLETIN DE LA SOCIÉTÉ MATHÉMATIQUE DE FRANCE 
Lemme 6.5. - Soit $f: Z \rightarrow Z^{\prime}$ un morphisme fini et plat de degré $\delta$ où $Z$ et $Z^{\prime}$ sont des variétés affines et lisses sur $k$ et $Z$ est intègre. On a le diagramme commutatif

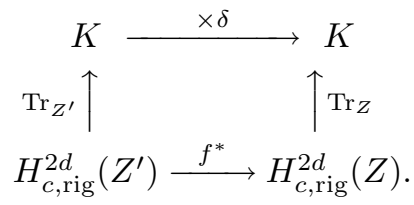

où d est la dimension de $Z$ et $Z^{\prime}$.

Démonstration. - D'après le théorème d'Elkik [11, th. 6], on peut trouver deux $C$-algèbres lisses $A$ et $A^{\prime}$ telles que $\mathscr{Z}=\operatorname{Spec}(A)$ et $\mathscr{Z}^{\prime}=\operatorname{Spec}\left(A^{\prime}\right)$ relèvent $Z$ et $Z^{\prime}$ respectivement. Il existe alors un morphisme fini et plat $\varphi: A^{\prime \dagger} \rightarrow A^{\dagger}$ entre les complétés faibles de $A^{\prime}$ et $A$ relevant $f$. Il existe alors [4, 2.5.3] des voisinages stricts $V$ et $V^{\prime}$ de $] Z\left[\mathscr{Z}_{K}^{\text {an }}\right.$ et $] Z^{\prime}{ }_{\mathscr{Z}^{\prime} \text { an }}^{\text {an }}$ et un morphisme $\varphi^{\text {an }}: V \rightarrow V^{\prime}$ qui prolonge $\varphi$. Quitte a restreindre $V^{\prime}$, on peut supposer que $\varphi^{\text {an }}$ est fini et plat. On sait qu'il existe [26, th 8.3$]$ un morphisme

$$
\operatorname{Tr}_{\varphi}: \Omega_{A^{\prime \dagger}}^{\star} \longrightarrow \Omega_{A^{\dagger}}^{\star}
$$

tel que, si on le compose avec le morphisme canonique $\Omega_{A^{\dagger}}^{\star} \rightarrow \Omega_{A^{\prime \dagger}}^{\star}$, on obtienne la multiplication par $\alpha$. Quitte à restreindre encore $V^{\prime}$, on peut, en procédant comme ci-dessus, obtenir un morphisme

$$
\operatorname{Tr}_{\varphi^{\text {an }}}: \varphi_{*}^{\text {an }} \Omega_{V}^{\star} \longrightarrow \Omega_{V^{\prime}}^{\star}
$$

qui, composé avec le morphisme $\Omega_{V^{\prime}}^{\star} \rightarrow \varphi_{*}^{\text {an }} \Omega_{V}^{\star}$, donne la multiplication par $\alpha$. De plus, le morphisme $\varphi^{\text {an }}$ étant fini, on a

$$
\mathbb{R} \varphi_{*}^{\mathrm{an}} \Omega_{V}^{\star}=\varphi_{*}^{\mathrm{an}} \Omega_{V}^{\star} .
$$

Le morphisme $\operatorname{Tr}_{\varphi^{\text {an }}}$ induit donc un morphisme

$$
\operatorname{Tr}_{\varphi}: H^{2 d}\left(V, \Omega_{V}^{\star}\right)=H^{2 d}\left(V^{\prime}, \mathbb{R} \varphi_{*}^{\mathrm{an}} \Omega_{V}^{\star}\right) \longrightarrow H^{2 d}\left(V^{\prime}, \Omega_{V^{\prime}}^{\star}\right) .
$$

Ce dernier nous donne un morphisme trace

$$
\operatorname{Tr}_{f}: H_{c, \text { rig }}^{2 d}(Z) \longrightarrow H_{c, \text { rig }}^{2 d}\left(Z^{\prime}\right),
$$

tel que $f^{*} \circ \operatorname{Tr}_{f}$ est la multiplication par $\alpha$. Il nous reste donc à montrer que le diagramme

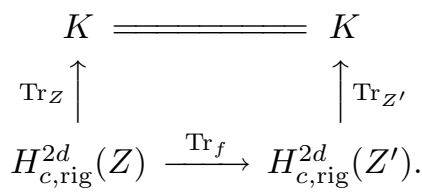

est commutatif. On regarde l'ensemble des points de $V$ où $\varphi^{\text {an }}$ n'est pas étale. C'est un fermé de Zariski de $V$ et sa projection par $\varphi^{\text {an }}$ qui est fini est donc un fermé de Zariski de $V^{\prime}$. De plus, $\varphi^{\text {an }}$ est génériquement étale. En effet $\operatorname{Frac}\left(\hat{A}^{\prime}{ }_{K}\right)$ est un corps de caractéristique 0 et $\hat{A}_{K}$ est réduite, $\operatorname{donc} \operatorname{Frac}\left(\hat{A}^{\prime}{ }_{K}\right) \otimes_{\hat{A}^{\prime}{ }_{K}} \hat{A}_{K}$ est étale sur $\operatorname{Frac}\left(\hat{A}^{\prime}{ }_{K}\right)$. Il existe donc un ouvert de Zariski non vide $W^{\prime} \subset V^{\prime}$ 
tel que $\varphi^{\text {an }}: W=\varphi^{\text {an-1 }}\left(W^{\prime}\right) \rightarrow W^{\prime}$ soit fini étale. On choisit $y$ un point de $W$ rationnel sur $K$, ce qui est possible après une extension finie de $K$, et on note $x \in Z$ sa spécialisation ainsi que $x^{\prime}$ l'image de ce dernier par $f$. On regarde les morphismes de Gysin $G_{x / Z}$ et $G_{x^{\prime} / Z^{\prime}}$. On sait $[6,1.3]$ que $\operatorname{Tr}_{Z} \circ G_{x / Z}=$ Id et $\operatorname{Tr}_{Z^{\prime}} \circ G_{x^{\prime} / Z^{\prime}}=$ Id. On est donc ramené à montrer que

$$
G_{x^{\prime} / Z^{\prime}}=\operatorname{Tr}_{f} \circ G_{x / Z} .
$$

On note $G_{y / V}$ (resp. $G_{y^{\prime} / V^{\prime}}$ ) le morphisme de Gysin $K_{y} \rightarrow \Omega_{V}^{\star}$ (respectivement $K_{y^{\prime}} \rightarrow \Omega_{V^{\prime}}^{\star}$ ), où $K_{y}$ (resp. $K_{y^{\prime}}$ ) désigne le faisceau constant sur $y$ (resp. sur $y^{\prime}$ ) de valeur $K$, obtenu à partir du morphisme de Gysin algébrique de $y$ dans $X$ (resp. $y^{\prime}$ dans $X^{\prime}$ ) par passage à l'analytique comme dans $[7,5]$. On est donc ramené à montrer que le diagramme

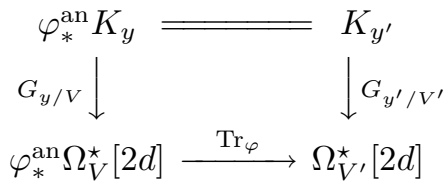

est commutatif. Soit $U^{\prime}$ un ouvert de $V^{\prime}$ contenant $y^{\prime}$ et $U=\left(\varphi^{\text {an }}\right)^{-1}\left(U^{\prime}\right)$, on a le diagramme commutatif :

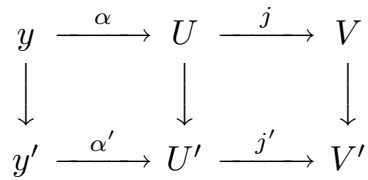

On a alors avec ces notations

$$
K_{y^{\prime}}=\left(j^{\prime} \circ \alpha^{\prime}\right)_{*} K_{y^{\prime}}=j_{!}^{\prime}\left(\alpha_{*}^{\prime} K_{y^{\prime}}\right) .
$$

Donc

$$
\operatorname{Hom}_{D\left(V^{\prime}\right)}\left(K_{y^{\prime}}, \Omega_{V^{\prime}}^{\star}[2 d]\right)=\operatorname{Hom}_{D\left(U^{\prime}\right)}\left(K_{y^{\prime}}, \Omega_{U^{\prime}}^{\star}[2 d]\right),
$$

où $D\left(V^{\prime}\right)$ (resp. $D\left(U^{\prime}\right)$ ) désigne la catégorie dérivée des faisceaux sur $V^{\prime}$ (resp. $U^{\prime}$ ). On peut donc localiser notre propos et supposer que $V^{\prime}$ est affinoïde et que $\varphi^{\text {an }}$ est fini étale. Quitte à faire une extension finie de $K$, on peut, de plus, supposer que la fibre $\left(\varphi^{\text {an }}\right)^{-1}\left(y^{\prime}\right)$ est constituée de $\alpha$ points $K$-rationnels. On peut donc, pour démontrer la commutativité du diagramme (19), passer au complété de $\Gamma\left(V^{\prime}, \mathscr{O}_{V^{\prime}}\right)$ par rapport à l'idéal maximal définissant $y^{\prime}$. On est donc ramené à montrer que, si $A^{\prime}$ est un anneau local noethérien régulier complet et $A=\bigoplus_{i=1}^{\alpha} A^{\prime}$, le diagramme

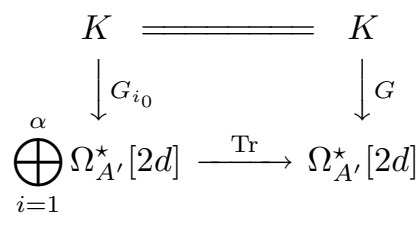

BULletin DE LA SOCIÉtÉ MATHÉMATIQUE DE FRANCE 
est commutatif, $G$ étant le morphisme de Gysin, $G_{i_{0}}$ le morphisme de Gysin dans la composante $i_{0}$ et le morphisme Tr étant induit par le morphisme $A \rightarrow A^{\prime}$ qui est la somme des $\alpha$ composantes. Ce dernier fait est alors clair.

La démonstration de la proposition 6.4 est donc finie.

6.2. Compatibilité à l'équivalence rationnelle. - Nous allons montrer que les classes de cycles définies ci-dessus, sont compatibles à l'équivalence rationnelle. Pour ce faire, nous aurons besoin de la trivialité de la classe d'un diviseur principal (principal triviality dans [10], [24]).

On se donne une $k$-variété lisse $X$ de dimension $n$ et $D$ un diviseur sur $X$. Soit $[D]=\sum n_{i} Z_{i}$ le diviseur de Weil associé. On peut définir la classe fondamentale de $[D]$ par linéarité :

$$
\eta_{[D]}:=\sum n_{i} \eta_{Z_{i}} \in \bigoplus H_{Z_{i}, \text { rig }}^{2}(X)=H_{|D|, \text { rig }}^{2}(X)
$$

où $|D|=\bigcup Z_{i}$ est le support du cycle $D$. Rappelons aussi que l'on peut associer à $D$ un pseudo-diviseur $\left(\mathscr{O}(D),|D|, s_{D}\right)$ et donc une classe de cohomologie que nous noterons $c_{1}(D) \in H_{|D| \text {,rig }}^{2}(X)$.

Nous allons comparer la classe fondamentale $\eta_{[D]}$ avec la classe $c_{1}(D) \mathrm{du}$ diviseur $D$.

Proposition 6.6. - Soient $X$ une variété lisse et $D$ un diviseur sur $X$. On a dans $H_{|D|, \text { rig }}^{2}(X)$, l'égalité

$$
\eta_{[D]}=c_{1}(D)
$$

Nous allons avoir besoin d'espaces analytiques. Nous allons donc reprendre la définition $[4,0.3]$.

Nous allons définir la structure analytique pour les variétés affines, le cas général s'obtenant par recollement.

Soit $\mathrm{Y}$ une $K$-variété affine. On pose $\mathrm{Y}=\operatorname{Spec}(A)$ et on se donne une présentation

$$
A=K\left[T_{1}, \ldots, T_{q}\right] /\left(f_{1}, \ldots f_{r}\right) .
$$

On pose alors $T_{i}^{(m)}=\pi^{m} T_{i}, f_{j}^{(m)}=f_{j}\left(\pi^{-m} T_{1}^{(m)}, \ldots, \pi^{-m} T_{q}^{(m)}\right)$ et

$$
\widehat{A}_{m}:=K\left\{T_{1}^{(m)}, \ldots, T_{q}^{(m)}\right\} /\left(f_{1}^{(m)}, \ldots, f_{r}^{(m)}\right) .
$$

On note $\mathrm{Y}^{f}$ l'ensemble des points fermés de $\mathrm{Y}$ et

$$
\mathrm{Y}_{m}=\left\{x \in \mathrm{Y}^{f} ;\left|T_{i}(x)\right| \leqslant|\pi|^{-m}\right\} \text {. }
$$

On a alors une bijection $\operatorname{Spm}\left(\widehat{A}_{m}\right) \rightarrow \mathrm{Y}_{m}$ qui munit ce dernier d'une structure d'espace analytique. On utilise pour finir que $\mathrm{Y}^{f}=\bigcup_{m} \mathrm{Y}_{m}$ pour munir $\mathrm{Y}^{f}$ d'une structure d'espace analytique. Cette structure ne dépend ni du choix de $\pi$ ni de celui de la présentation. On notera $Y^{\text {an }}$ la variété analytique ainsi définie.

TOME $131-2003-\mathrm{N}^{\mathrm{O}} 1$ 
REMARQUE 6.7. - Il faut faire attention que si la structure de variété analytique ainsi définie ne dépend pas du choix de la présentation les ouverts $\mathrm{Y}_{m}$, eux, en dépendent.

Il existe aussi un foncteur faisceau analytique :

$$
\begin{aligned}
\operatorname{Mod}(\mathrm{Y}) & \longrightarrow \operatorname{Mod}\left(\mathrm{Y}^{\mathrm{an}}\right), \\
\mathscr{F} & \longmapsto \mathscr{F}^{\text {an }}
\end{aligned}
$$

où $\operatorname{Mod}(\mathrm{Y})$ (resp. $\left.\operatorname{Mod}\left(\mathrm{Y}^{\mathrm{an}}\right)\right)$ désigne la catégorie des faisceaux en $\mathscr{O}_{\mathrm{Y}}$ (resp. $\mathscr{O}_{\text {Yan }}$ ) modules sur $\mathrm{Y}$ (resp. $\left.\mathrm{Y}^{\mathrm{an}}\right)$. On peut décrire ce foncteur de la manière suivante. Pour tout ouvert $U$ de $Y, U^{\text {an }}$ est un ouvert de $Y^{\text {an }}$ et on a

$$
\mathscr{F}^{\text {an }}\left(\mathrm{U}^{\mathrm{an}}\right)=\mathscr{F}(\mathrm{U}) \otimes_{\mathscr{O}_{\mathrm{Y}(\mathrm{U})}} \mathscr{O}_{\mathrm{Yan}}\left(\mathrm{U}^{\mathrm{an}}\right) .
$$

Or, comme $\mathscr{O}_{\text {Yan }}$ est plat sur $\mathscr{O}_{Y}$, on a :

Proposition 6.8. - Le foncteur faisceau analytique associé est exact.

Démonstration de la proposition 6.6. - Par linéarité, on peut supposer que $D$ est le diviseur associé à un sous-schéma fermé intègre $Z$. De plus la classe fondamentale et la classe d'un diviseur étant compatibles à l'extension des scalaires (propositions 2.6 et 3.15) , il nous suffit de démontrer cette égalité après une extension finie du corps de base.

On peut donc utiliser les lemmes 6.2 et 6.3 . Soit $V$ un ouvert lisse de $Z$. On note $T=Z-V$ et $U=X-T$.

Via l'isomorphisme $H_{|D|, \text { rig }}^{2}(X) \stackrel{\sim}{\rightarrow} H_{\left|D_{U}\right| \text {,rig }}^{2}(U)$, on a

$$
c_{1}(D)=c_{1}\left(D_{U}\right) \quad \text { et } \quad \eta_{[D]}=\eta_{\left[D_{U}\right]} .
$$

Quitte à ôter d'autres fermés, on peut, comme dans [7, 3.7], supposer de plus que $Z$ et $X$ sont affines et lisses et qu'il existe deux $C$-schémas $\mathscr{Z}$ et $\mathscr{X}$ affines et lisses ainsi qu'une immersion fermée de $\mathscr{Z}$ dans $\mathscr{X}$ telle que l'on obtienne l'inclusion de $Z$ dans $X$ en passant aux fibres spéciales. Quitte à localiser encore, on peut de plus supposer qu'il existe $t \in \Gamma\left(\mathscr{X}, \mathscr{O}_{\mathscr{X}}\right)$ tel que $\mathscr{Z}=V(t)$. On note respectivement $Z_{K}$ et $X_{K}$ les fibres génériques de $\mathscr{Z}$ et $\mathscr{X}$.

On sait alors grâce au lemme 2.8 que la classe fondamentale est l'image de 1 par le morphisme de Gysin. On va exprimer explicitement le morphisme de Gysin algébrique de l'immersion $\mathscr{Z} \hookrightarrow \mathscr{X}$ en reprenant la construction de [7]. On sait d'après [1, VI.3] qu'il existe, dans la catégorie dérivée, un morphisme de Gysin en cohomologie de de Rham :

$$
G_{\mathscr{Z} / \mathscr{X}}: \Omega_{\mathscr{Z}}^{\bullet} \longrightarrow \Omega_{\mathscr{X}}^{\bullet}[2] .
$$

Nous allons expliciter ce morphisme en tant que morphisme de complexes. Commençons par noter $\mathscr{H}_{\mathscr{Z}}^{1}\left(\Omega_{\mathscr{X}}^{\bullet}\right)$ le complexe :

$$
0 \rightarrow \mathscr{H}_{\mathscr{Z}}^{1}\left(\mathscr{O}_{\mathscr{X}}\right) \rightarrow \mathscr{H}_{\mathscr{Z}}^{1}\left(\Omega_{\mathscr{X}}^{1}\right) \rightarrow \cdots \rightarrow \mathscr{H}_{\mathscr{Z}}^{1}\left(\Omega_{\mathscr{X}}^{i}\right) \rightarrow \cdots
$$

où $\mathscr{H}_{\mathscr{Z}}^{1}$ désigne le premier faisceau de cohomologie à support dans $\mathscr{Z}$. 
On définit un morphisme

$$
a: \Omega_{\mathscr{Z}}^{\bullet} \longrightarrow \mathscr{H}_{\mathscr{Z}}^{1}\left(\mathscr{O}_{\mathscr{X}}\right) \otimes \Omega_{\mathscr{X}}^{\bullet}[1]
$$

en posant, pour tout ouvert $W$, tout $\alpha$ et tout $\omega \in \Gamma\left(W, \Omega_{\mathscr{Z}}^{\alpha}\right)$

$$
a(\omega)=\frac{\widetilde{\omega}}{t} \wedge \mathrm{d} t \in \Gamma\left(W, \mathscr{H}_{\mathscr{Z}}^{1}\left(\mathscr{O}_{\mathscr{X}}\right) \otimes \Omega_{\mathscr{X}}^{\alpha+1}\right) .
$$

où $\widetilde{\omega}$ est un relèvement de $\omega$ dans $\Gamma\left(W, \Omega_{\mathscr{X}}^{\alpha}\right)$.

Maintenant, pour tout faisceau de $\mathscr{O}_{\mathscr{X}}$-modules $\mathscr{F}$ plat sur $\mathscr{O}_{\mathscr{X}}$, on a la résolution $\mathscr{M}^{\star} \mathscr{F}$ suivante $[19,2.2 .2]$ :

$$
\mathscr{F} \rightarrow \mathscr{M}^{0} \mathscr{F}=i_{*}\left(i^{*} \mathscr{F}\right) \longrightarrow \mathscr{M}^{1} \mathscr{F}=\mathscr{H}_{\mathscr{Z}}^{1} \mathscr{F}
$$

où $i: \mathscr{X}-\mathscr{Z} \hookrightarrow \mathscr{X}$ est l'immersion ouverte canonique.

Le morphisme de Gysin est alors le morphisme suivant :

$$
\Omega_{\mathscr{Z}}^{\bullet} \stackrel{a}{\longrightarrow} \mathscr{H}_{\mathscr{Z}}^{1}\left(\mathscr{O}_{\mathscr{X}}\right) \otimes \Omega_{\mathscr{X}}^{\bullet}[1] \stackrel{b}{\longrightarrow} \mathscr{M}^{\star} \Omega_{\mathscr{X}}^{\bullet}[2] \stackrel{\sim}{\longleftarrow} \Omega_{\mathscr{X}}^{\bullet}[2],
$$

où $b$ est le morphisme évident.

On considère le morphisme induit sur la fibre générique. On passe ensuite aux variétés analytiques associées. On obtient alors le morphisme dans la catégorie des complexes de faisceaux en $K$-vectoriels sur $X_{K}^{\text {an }}$

$$
G_{Z_{K} / X_{K}}^{\text {an }}: \Omega_{Z_{K}^{\text {an }}}^{\longrightarrow} \longrightarrow \mathscr{H}_{Z_{K}}^{1}\left(\mathscr{O}_{X_{K}}\right)^{\text {an }} \otimes \Omega_{X_{K}^{\text {an }}}^{\bullet}[1] \longrightarrow \mathscr{M}^{\star} \Omega_{X_{K}^{\text {an }}}^{\bullet}[2]
$$

où la résolution $\mathscr{M}$ est obtenue en appliquant le foncteur exact faisceau analytique associé à (20).

On se donne maintenant $(\overline{\mathscr{X}}, j)$ une compactification de $\mathscr{X}$ sur $C$ et on note $\overline{\mathscr{Z}}$ l'adhérence schématique de $\mathscr{Z}$ dans $\overline{\mathscr{X}}$. On note alors $\bar{X}$ (resp. $\bar{Z}$ ), $\bar{X}_{K}\left(\right.$ resp. $\left.\bar{Z}_{K}\right)$ et $\widehat{\mathscr{X}}$ (resp. $\widehat{\mathscr{Z}}$ ) la fibre spéciale, la fibre générique et le complété formel de $\overline{\mathscr{X}}$ (resp. $\overline{\mathscr{Z}}$ ). Comme $\overline{\mathscr{X}}$ et $\overline{\mathscr{Z}}$ sont propres sur $C$, on a

$$
\widehat{\overline{\mathscr{X}}}_{K}=\bar{X}_{K}^{\text {an }} \quad \text { et } \quad \widehat{\mathscr{Z}}_{K}=\bar{Z}_{K}^{\text {an }} \text {. }
$$

Le morphisme de Gysin rigide est obtenu en appliquant le foncteur $\Gamma_{] \bar{Z}[}^{\dagger} j^{\dagger}$ (voir $[7,5.4])$ à $G_{Z_{K} / X_{K}}^{\text {an }}$.

On obtient un morphisme de complexes de faisceaux de $K$-vectoriels sur $\bar{X}_{K}^{\text {an }}$

$$
\Gamma_{] \bar{Z}[}^{\dagger} j^{\dagger} \Omega_{\bar{Z}_{K}^{\text {an }}}^{\bullet} \longrightarrow \Gamma_{] \bar{Z}[}^{\dagger} j^{\dagger}\left(\mathscr{H}_{\bar{Z}_{K}}^{1}\left(\mathscr{O}_{\bar{X}_{K}}\right)^{\text {an }} \otimes \Omega_{\bar{X}_{K}^{\text {an }}}[1]\right) \longrightarrow \Gamma_{] \bar{Z}[}^{\dagger} j^{\dagger}\left(\mathscr{M}^{\star} \Omega_{\bar{X}_{K}^{\text {an }}}[2]\right) \text {. }
$$

Or, les foncteurs $j^{\dagger}$ et $\Gamma_{] \bar{Z}[}^{\dagger}$ sont exacts, donc $\Gamma_{] \bar{Z}[}^{\dagger} j^{\dagger}\left(\mathscr{M}^{\star} \Omega_{\bar{X}_{K}^{\text {an }}}\right)$ est quasiisomorphe à $\Gamma_{]}^{\dagger} j_{[}^{\dagger}\left(\Omega_{\bar{X}_{K}^{\text {an }}}^{\bullet}\right)$.

On considère un recouvrement $\mathfrak{U}$ de $\overline{\mathscr{X}}$ tel que sur chaque ouvert $\overline{\mathscr{U}}_{i}$ du recouvrement, le fermé $\overline{\mathscr{Z}}_{i}$ soit défini par l'annulation d'une section $t_{i}$. On regarde alors nos deux classes comme des éléments de

$$
H^{2}\left(\mathfrak{U},\left(j_{X}^{\dagger} \mathscr{M}^{\star} \Omega_{X}^{\star} \text { an } \rightarrow j_{U}^{\dagger} \mathscr{M}^{\star} \Omega_{X}^{\star} \text { an }\right)_{s}\right),
$$

TOME $131-2003-\mathrm{N}^{\mathrm{O}} 1$ 
où $U=X-Z$.

On considère pour finir

$$
\zeta \in C^{0}\left(\mathfrak{U}, j_{X}^{\dagger} \mathscr{M}^{0} \Omega_{X_{K}^{\mathrm{an}}}^{1}\right) \subset C^{1}\left(\mathfrak{U},\left(j_{X}^{\dagger} \mathscr{M}^{\star} \Omega_{X_{K}^{\mathrm{an}}}^{\star} \rightarrow j_{U}^{\dagger} \mathscr{M}^{\star} \Omega_{X_{K}^{\mathrm{an}}}^{\star}\right)_{s}\right)
$$

défini par

$$
\zeta_{i}=\frac{\mathrm{d} t_{i}}{t_{i}}
$$

Si on note $\Delta$ la différentielle du complexe $C^{\star}\left(\mathfrak{U},\left(j_{X}^{\dagger} \mathscr{M}^{\star} \Omega_{X_{K}^{\star a n}}^{\star} \rightarrow j_{U}^{\dagger} \mathscr{M}^{\star} \Omega_{X_{K}^{\star a n}}^{\star}\right)_{s}\right)$, on a

$$
\Delta(\zeta)=c_{1}(D)-G_{Z / X}^{\mathrm{rig}}(1)
$$

En effet, si $\delta$ est la différentielle provenant de la différentielle de $C^{\star}$, d celle qui provient de la différentielle $j_{X}^{\dagger} \mathscr{M}^{\star} \Omega_{X_{K}^{\star}}^{\star} \stackrel{\mathrm{d}}{\longrightarrow} j_{U}^{\dagger} \mathscr{M}^{\star} \Omega_{X_{K}^{\star}}^{\text {an }}$ et $\mathrm{d}^{\prime}$ celle qui provient de celle de $\mathscr{M}^{\star}$, on a

$$
\begin{aligned}
\delta(\zeta) & =\frac{\mathrm{d} t_{j} / t_{i}}{t_{j} / t_{i}} \in C^{1}\left(\mathfrak{U}, j_{X}^{\dagger} \mathscr{M}^{0} \Omega_{X_{K}^{\text {an }}}^{1}\right), \quad \mathrm{d}(\zeta)=\frac{\mathrm{d} t_{i}}{t_{i}} \in C^{0}\left(\mathfrak{U}, j_{U}^{\dagger} \mathscr{M}^{0} \Omega_{X_{K}^{\mathrm{an}}}^{1}\right) \quad \text { et } \\
\mathrm{d}^{\prime}(\zeta) & =G_{Z / X}^{\mathrm{rig}}(1) \in C^{0}\left(\mathfrak{U}, j_{X}^{\dagger} \mathscr{M}^{1} \Omega_{X_{K}^{\mathrm{an}}}^{1}\right) .
\end{aligned}
$$

Corollaire 6.9 (principal triviality). — Soit $i:|D| \hookrightarrow X$ un diviseur principal dans une variété lisse. On a

$$
i_{*} \eta_{[D]}=0
$$

Démonstration. - On a

$$
i_{*} \eta_{[D]}=i_{*} c_{1}(D)=c_{1}(\mathscr{O}(D)) .
$$

Or $D$ est principal donc $\mathscr{O}(D)$ est trivialisable sur $X$.

On peut maintenant montrer que les classes de cycles sont compatibles à l'équivalence rationnelle.

Proposition 6.10. - Soient $X$ une $k$-variété et $z \in Z(X)$ un cycle rationnellement équivalent à zéro. On a

$$
\gamma(z)=0 \text {. }
$$

L'application classe de cycle passe donc au quotient par l'équivalence rationnelle et définit de la sorte

$$
\gamma: A_{\bullet}(X) \longrightarrow H_{\bullet}^{\text {rig }}(X) .
$$

Démonstration. - Il suffit de montrer que, si on se donne sur $X$ un diviseur de Cartier principal $D$, on a $\gamma([D])=0$. Là encore, grâce à la compatibilité des classes de cycles aux extensions finies du corps de base, nous allons pouvoir utiliser des techniques d'excisions (lemme 6.3). 
Commençons par montrer que l'on peut supposer que $X$ est normale. En effet, soit $\pi: X^{\prime} \rightarrow X$ la normalisation de $X$. On sait $[13,2.3]$ que

$$
\pi_{*}\left[\pi^{*} D\right]=[D],
$$

où $\pi^{*} D$ est bien défini car $\pi\left(X^{\prime}\right)=X \nsubseteq|D|$. Donc d'après ce qui précède,

$$
\gamma([D])=\pi_{*} \gamma\left(\left[\pi^{*} D\right]\right) .
$$

De plus, $\pi^{*} D$ est aussi principal.

On suppose donc $X$ normale de dimension $n$. On sait alors qu'il existe $T$ un sous-schéma fermé de codimension supérieure ou égale à deux tel que $U=X-T$ et $Z=[D] \cap U$ soient lisses. On a le diagramme commutatif :

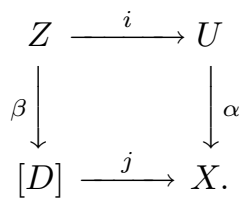

Grâce au théorème 2.10 et au corollaire 6.9 , on a

$$
\alpha^{*} \gamma([D])=\alpha^{*} j_{*} \eta_{[D]}=i_{*} \beta^{*} \eta_{[D]}=i_{*} \eta_{Z}=0 .
$$

Maintenant en utilisant la suite exacte longue

$$
\cdots H_{2 n-2}^{\mathrm{rig}}(T) \longrightarrow H_{2 n-2}^{\mathrm{rig}}(X) \stackrel{\alpha^{*}}{\longrightarrow} H_{2 n-2}^{\mathrm{rig}}(U) \rightarrow \cdots
$$

et sachant que $H_{2 n-2}^{\text {rig }}(T)=0$, on voit que $\alpha^{*}: H_{2 n-2}^{\text {rig }}(X) \rightarrow H_{2 n-2}^{\text {rig }}(U)$ est injective, donc $\gamma([D])=0$.

\section{Intersection de cycles et applications}

Nous allons voir que les classes de cycles définies précédemment sont compatibles à l'intersection. Nous reprenons ici en grande partie les arguments de $[13,19]$. Nous regarderons d'abord le cas où la variété ambiante est lisse avant d'étudier le cas des variétés singulières.

7.1. Variétés lisses. — Rappelons que si $i: Z \rightarrow X$ est une immersion d'une variété de dimension $d$ dans une variété lisse de dimension $n$, on note $c(i) \in H_{Z \text {,rig }}^{2(n-d)}(X)$ l'antécédent par le morphisme de dualité de Poincaré de la classe fondamentale $\eta_{Z} \in H_{2 d}^{\mathrm{rig}}(Z)$.

Commençons par un lemme :

TOME $131-2003-\mathrm{N}^{\mathrm{O}} 1$ 
LEMME 7.1. - Soit le carré cartésien

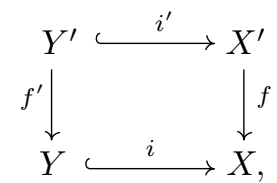

où $X$ et $X^{\prime}$ sont deux variétés lisses et $i$ et $i^{\prime}$ deux immersions fermées régulières de codimensions $r$.

1) On a $f^{*} c(i)=c\left(i^{\prime}\right)$, dans $H_{Y^{\prime}, \text { rig }}^{2 r}\left(X^{\prime}\right)$ où $c(i)$ est la classe définie au paragraphe 2.3 .

2) Si de plus, $f$ et $f^{\prime}$ sont aussi des immersions régulières de codimensions e,

$$
c(i) \cup c(f)=c(f) \cup c(i)=c\left(f i^{\prime}\right)=c\left(i f^{\prime}\right)
$$

dans $H_{Y^{\prime}}^{2 r+2 e}(X)$.

Démonstration. - 1) L'immersion $i$ étant régulière, on peut, quitte à se limiter à un ouvert à l'aide du théorème de pureté, supposer que $i$ est la composée de $d$ immersions de codimension 1 . On est donc ramené au cas de la codimension 1. Le lemme découle alors du lemme suivant dont l'analogue en cohomologie de de Rham se trouve dans [21, 7.7.2] et dont la démonstration se transpose dans notre cas.

Lemme 7.2. - Soient $f: X^{\prime} \rightarrow X$ un morphisme de variétés intègres avec $X$ lisse et $n^{\prime}$ la dimension de $X^{\prime}$. Pour tout diviseur de Cartier $D$ sur $X$ tel que $f\left(X^{\prime}\right) \nsubseteq|D|$, on a dans $H_{2 n^{\prime}-2}^{\mathrm{rig}}\left(X^{\prime}\right)$

$$
\eta_{X^{\prime}} \cap f^{*} c_{1}(D)=\eta_{\left[f^{*} D\right]} .
$$

2) La démonstration est similaire à celle de la première partie. Après une réduction au cas où $i$ est de codimension 1 , on applique le lemme 7.2 puis la formule de projection.

REMARQUe 7.3. - La deuxième partie du lemme dit que le produit des classes de deux cycles de codimensions $e$ et $r$ qui se coupent proprement, c'est-à-dire que toutes les composantes de l'intersection sont de codimension $r+e$, est la classe de l'intersection des cycles.

Pour traiter le cas général on aura besoin aussi du lemme suivant :

Lemme 7.4. - Soient $X$ une variété lisse et $i: X \hookrightarrow N$ la section nulle d'un fibré vectoriel $N$ de rang $d$. Pour tout $k$-cycle $\alpha$ de $N$, on a

$$
c(i) \cap \gamma_{N}(\alpha)=\gamma_{X}\left(i^{*} \alpha\right)
$$

où $i^{*}$ est le morphisme de Gysin. 
Démonstration. — On sait $[13,3.3]$ que si on note $\pi: N \rightarrow X$ le morphisme structural de $N$, on a

$$
\pi^{*}: A_{k-d}(X) \stackrel{\sim}{\longrightarrow} A_{k}(N) .
$$

On peut donc supposer que $\alpha=\pi^{*} u$. De plus par linéarité, on peut supposer que $u$ est un sous-schéma fermé intègre $Z$. Grâce au lemme ci-dessus, on se ramène au cas où $Z=X$. Dès lors, notre propriété est la dualité de Poincaré

$$
c(i) \cap \eta_{N}=\eta_{X}
$$

Nous aurons alors besoin de la proposition suivante :

Proposition 7.5. - Soient $X$ et $Y$ deux variétés lisses et $f: X \rightarrow Y$ une immersion fermée. Pour tout cycle $\alpha \in A_{k}(Y)$, on a

$$
\gamma_{X}\left(f^{*} \alpha\right)=c(f) \cap \gamma_{Y}(\alpha)
$$

Démonstration. - Par linéarité, on peut supposer que $\alpha$ est la classe d'un sousschéma fermé intègre de $Y$ que nous noterons $V$. Dès lors on pose $W=X \times_{Y} V$. On note alors $N$ le fibré normal de $X$ dans $Y$. On pose $M$ l'éclaté de $X \times \infty$ dans $Y \times \mathbb{P}^{1}$ d'où on a ôté le diviseur exceptionnel. On sait alors $[13,5]$ que l'on a le diagramme commutatif suivant :

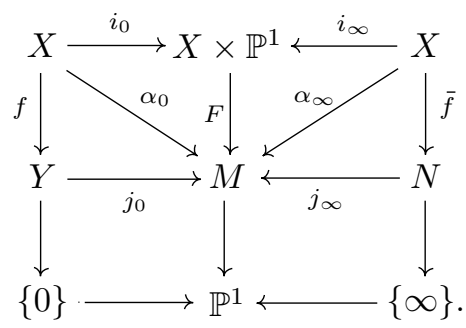

Si on note $M^{\prime}$ la déformation similaire définie pour l'immersion de $W$ dans $V$, on notera $G$ l'immersion de $M^{\prime}$ dans $M$. En appliquant le lemme 7.2 au morphisme $G: M^{\prime} \rightarrow M$ et au diviseur $Y$, on obtient

$$
\eta_{M^{\prime}} \cap G^{*} c\left(j_{0}\right)=\eta_{V} .
$$

On a donc, grâce à la formule de projection

$$
\begin{array}{rlrl}
c(f) \cap \gamma_{Y}([V]) & =c(f) \cap g_{*}\left(\eta_{M^{\prime}} \cap G^{*} c\left(j_{0}\right)\right) & & \\
& =c(f) \cap\left(g_{*}\left(\eta_{M^{\prime}}\right) \cap c\left(j_{0}\right)\right) & & \text { (formule de projection) } \\
& =j_{0}^{*} c(F) \cap\left(g_{*}\left(\eta_{M^{\prime}}\right) \cap c\left(j_{0}\right)\right) & & \text { (lemme 7.1) } \\
& =\left(c(F) \cup c\left(j_{0}\right)\right) \cap\left(g_{*}\left(\eta_{M^{\prime}}\right)\right. & \\
& =c\left(i_{0}\right) \cap\left(g_{*}\left(\eta_{M^{\prime}}\right) \cap c(F)\right) . &
\end{array}
$$

TOME $131-2003-\mathrm{N}^{\mathrm{O}} 1$ 
Maintenant, avec les notations ci-dessus, soit $a \in H_{*}\left(X \times \mathbb{P}^{1}\right)$. On a

$$
c\left(i_{0}\right) \cap a=c\left(i_{\infty}\right) \cap a .
$$

En effet, si on note $p$ la projection $X \times \mathbb{P}^{1} \rightarrow \mathbb{P}^{1}$, on a

$$
c\left(i_{0}\right) \cap a=p^{*} c(\{0\}) \cap a,
$$

où $c(0)$ est la classe de l'immersion de $\{0\}$ dans $\mathbb{P}^{1}$. Notre égalité vient du fait que cette dernière est égale à la classe de l'immersion de $\{\infty\}$ dans $\mathbb{P}^{1}$. On a donc

$$
c(f) \cap \gamma_{Y}([V])=c\left(i_{\infty}\right) \cap\left(g_{*}\left(\eta_{M^{\prime}}\right) \cap c(F)\right)
$$

et en refaisant le calcul précédent à l'envers, on a

$$
c(f) \cap \gamma_{Y}([V])=c(\bar{f}) \cap \gamma_{N}([C])
$$

où $[C]$ est le cône de $X \times_{Y} V$ dans $V$. On termine la démonstration en utilisant le lemme 7.4 .

Corollaire 7.6. - Soient $X$ une variété lisse, $x \in A^{k}(X)$ et $y \in A^{\ell}(X)$ deux cycles. On a dans $H_{\text {rig }}^{2(k+\ell)}(X)$ :

$$
\gamma_{X}(x \cdot y)=\gamma_{X}(x) \cup \gamma_{X}(y) .
$$

C'est-à-dire, le morphisme

$$
\gamma_{X}: A^{\bullet}(X) \longrightarrow H_{\text {rig }}^{\bullet}(X)
$$

est un morphisme d'anneau.

Démonstration. - On peut supposer que $x$ et $y$ sont représentés par des sousschémas intègres $Z_{x}$ et $Z_{y}$. Par réduction à la diagonale [16, II.4.2.19], on se ramène à montrer que

$$
\gamma_{X}\left(\Delta^{*}\left(Z_{x} \times_{k} Z_{y}\right)\right)=c(\Delta) \cup \gamma_{X \times{ }_{k} X}\left(Z_{x} \times_{k} Z_{y}\right),
$$

où $\Delta: X \hookrightarrow X \times_{k} X$ est la diagonale. Cette dernière égalité est la conclusion de la proposition précédente.

On sait de plus que pour tout morphisme $f: X^{\prime} \rightarrow X$ entre deux variétés lisses, il existe un morphisme de fonctorialité $f^{*}: A^{\bullet}(X) \rightarrow A^{\bullet}\left(X^{\prime}\right)$ défini de la manière suivante. En notant $p: X \times X^{\prime} \rightarrow X$ et $p^{\prime}: X \times X^{\prime} \rightarrow X^{\prime}$ les deux projections et $\Gamma_{f}$ le graphe de $f$, on pose, pour tout cycle $Z$ de $X^{\prime}$,

$$
f^{*}(Z)=p_{*}^{\prime}\left(\Gamma_{f} \cdot p^{-1}\left(Z^{\prime}\right)\right) .
$$

Proposition 7.7. - Soit $f: X^{\prime} \rightarrow X$ un morphisme entre deux variétés lisses. On a pour tout $z \in A^{\bullet}(X)$

$$
f^{*} \gamma_{X}(z)=\gamma_{X^{\prime}} f^{*} z
$$

où $\gamma_{X}$ et $\gamma_{X^{\prime}}$ désignent les applications classes de cycles. 
Démonstration. - On suppose d'abord que $f$ est plat. On peut supposer que $z$ est la classe d'un sous-schéma fermé. Par excision on peut de plus supposer que $Z$ est lisse. On se ramène alors par une récurrence au cas de codimension 1 . Ce dernier découle du lemme 7.2.

Pour $f$ quelconque, on peut aussi supposer que $z$ est la classe d'un sousschéma fermé. On a alors :

$$
\begin{aligned}
& f^{*} \gamma_{X}(Z)=\Gamma_{f}^{*} p^{*} \gamma_{X}(Z) \quad=\Gamma_{f}^{*} \gamma_{X^{\prime} \times X}\left(p^{*} Z\right) \\
& =p_{*}^{\prime}\left(\Gamma_{f}\right)_{*} \Gamma_{f}^{*} \gamma_{X^{\prime} \times X}\left(p^{*} Z\right)=p_{*}^{\prime}\left(\gamma_{X^{\prime} \times X}\left(\Gamma_{f}\right) \cdot \gamma_{X^{\prime} \times X}\left(p^{*} Z\right)\right) \\
& =\gamma_{X^{\prime}}\left(p_{*}^{\prime}\left(\Gamma_{f} \cdot p^{*} Z\right)\right)=\gamma_{X^{\prime}}\left(f^{*} Z\right) \text {. }
\end{aligned}
$$

7.2. Cas général. - Soit $X$ une $k$-variété éventuellement singulière admettant une immersion dans une variété lisse. On note $A^{\bullet}(X)$ l'anneau de cohomologie de Chow.

On peut alors construire $\gamma: A^{\bullet} X \rightarrow H_{\text {rig }}^{\bullet}(X)$. En effet, soit $(Y, f)$ un objet de $\mathscr{C}(X)$; on a

$$
A^{\bullet} Y \stackrel{\gamma}{\longrightarrow} H_{\text {rig }}^{\bullet}(Y) \stackrel{f^{*}}{\longrightarrow} H_{\text {rig }}^{\bullet}(X) .
$$

La proposition 7.7 nous montre alors que ces flèches définissent bien le morphisme voulu.

On a alors :

Proposition 7.8. - Soient $X$ une variété, $x \in A_{p} X$ et $y \in A^{q} X$. On a

$$
\gamma(x \cap y)=\gamma(x) \cap \gamma(y) .
$$

Démonstration. - On choisit un représentant de $y$, à savoir un morphisme $f: X \rightarrow Y$ où $Y$ est une variété lisse et un cycle $y$ dans $A^{q}(Y)$. On doit montrer que

$$
\gamma_{X}\left(x \bullet_{f} y\right)=\gamma_{X}(x) \cap f^{*} \gamma_{Y}(y)
$$

On peut donc se restreindre par linéarité au cas où $x$ et $y$ sont représentés par des sous-schémas fermés intègres $Z_{X}$ et $Z_{Y}$. On se donne de plus, une immersion fermée de $X$ dans une variété lisse $V$. On a donc le diagramme commutatif suivant :

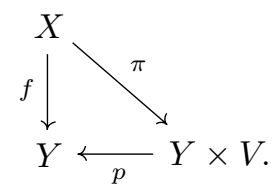

En utilisant la proposition 7.7, l'égalité (21) se ramène à

$$
\gamma_{X}\left(Z_{X} \bullet \pi p^{*}\left(Z_{Y}\right)\right)=\gamma_{X}\left(Z_{X}\right) \cap \pi^{*} \gamma_{Y \times V}\left(p^{*} Z_{Y}\right) .
$$

Cette dernière égalité est une conséquence du corollaire 7.6.

TOME $131-2003-\mathrm{N}^{\mathrm{O}} 1$ 
7.3. Applications. - Nous allons maintenant exhiber quelques conséquences du formalisme exposé précédemment (Théorème de Riemann-RochGrothendieck, formule de self-intersection, cohomologie d'un éclaté et action du Frobenius). Nous ne ferons pas de démonstrations : tous les résultats se déduisent directement de résultats analogues pour les groupes de Chow. On pourra cependant consulter $[31,5]$ ou $[30,6]$.

Pour commencer, nous allons rappeler la définition du polynôme de Chern et du polynôme de Todd [SGA6], $[22]$. On se place dans $\mathbb{Q}\left[\left[T_{i}\right]\right]_{i \in \mathbb{N}}$ des séries formelles à nombre dénombrable de variables. On note $C_{i}$ les fonctions symétriques des variables. Le polynôme de Chern est défini par

$$
\operatorname{ch}\left(C_{i}\right)=\sum_{i \in \mathbb{N}} \exp \left(T_{i}\right)
$$

où $\exp x=\sum_{i=0}^{\infty} x^{n} / n$ !. De même, le polynôme de Todd est défini par

$$
\operatorname{td}\left(C_{i}\right)=\prod_{i \in \mathbb{N}} \frac{T_{i}}{1-\exp \left(-T_{i}\right)} .
$$

Soient $X$ une $k$-variété et $\mathscr{E}$ un faisceau localement libre de $\operatorname{rang} r \operatorname{sur} X$. On définit le polynôme de Chern et la classe de Todd de $\mathscr{E}$ par

$$
\begin{aligned}
\operatorname{ch}(\mathscr{E}) & :=\operatorname{ch}\left(c_{1}(\mathscr{E}), \ldots, c_{r}(\mathscr{E}), 0, \ldots\right) \\
\operatorname{td}(\mathscr{E}) & :=\operatorname{td}\left(c_{1}(\mathscr{E}), \ldots, c_{r}(\mathscr{E}), 0, \ldots\right)
\end{aligned}
$$

Si $X$ est lisse, on appelle classe de Todd de $X$ et on note $\operatorname{Td}(X)$ la classe de Todd du fibré tangent.

On peut maintenant énoncer un théorème de Riemann-Roch en cohomologie rigide.

THÉORÈme 7.9 (théorème de Riemann-Roch-Grothendieck)

Il existe une transformation naturelle $\tau: K_{0} \rightarrow H_{\bullet}^{\text {rig }}$ de foncteurs covariants de la catégorie des $k$-variétés quasi-projectives avec des morphismes propres dans la catégorie des groupes abéliens vérifiant:

1) Pour tout $X$, le diagramme

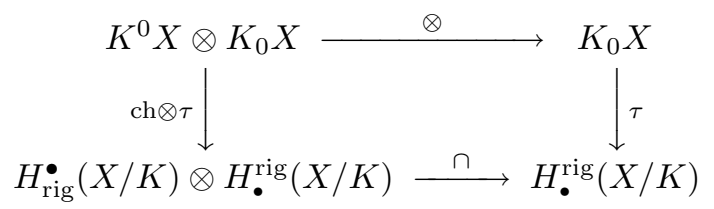

est commutatif.

2) Si $X$ est une $k$-variété lisse,

$$
\tau\left(\mathscr{O}_{X}\right)=\operatorname{Td}(X)
$$

BULlETIN DE LA SOCIÉtÉ MATHÉMATIQUE DE FRANCE 
3) Si j:U $\rightarrow X$ est une immersion ouverte de $k$-variétés, le diagramme

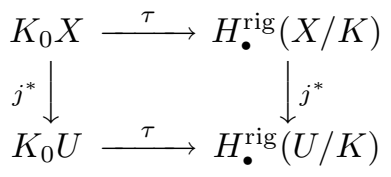

est commutatif.

Corollaire 7.10. - Soit $f: X \rightarrow Y$ un morphisme propre entre deux $k$ variétés lisses quasi-projectives. On a pour tout $\mathscr{E}$ dans $K^{0} X$ :

$$
f_{*}(\operatorname{ch}(\mathscr{E}) \cdot \operatorname{Td}(X))=\operatorname{ch}\left(f_{*}(\mathscr{E})\right) \cdot \operatorname{Td}(Y) .
$$

Soit $X$ une $k$-variété lisse et $Y$ un sous-schéma fermé lisse de codimension $d$. On note $\mathscr{J}$ l'idéal de $Y$ dans $X$ et $\mathscr{N}=\mathscr{J} / \mathscr{J}^{2}$.

ThÉorغ̀me 7.11 (formule de self-intersection). - Pour tout $y \in H_{\text {rig }}^{*}(Y)$, on $a$

$$
i^{*} i_{*}(y)=y \cdot c_{d}(\check{\mathscr{N}}) .
$$

On garde les notations précédentes. On considère $f: X^{\prime} \rightarrow X$ l'éclaté de $X$ le long de $Y$. On a alors le diagramme cartésien suivant :

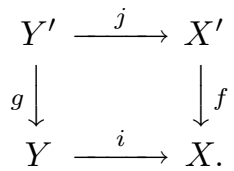

Pour tout $n \in \mathbb{N}$ on considère l'application

$$
\begin{gathered}
\psi_{n}: H_{\text {rig }}^{n}(X) \oplus H_{\text {rig }}^{n-2}\left(Y^{\prime}\right) \longrightarrow H_{\text {rig }}^{n}\left(X^{\prime}\right) \oplus H_{\text {rig }}^{n-2 d}(Y), \\
\text { resp. } \psi_{Y, n}: H_{Y, \text { rig }}^{n}(X) \oplus H_{\text {rig }}^{n-2}\left(Y^{\prime}\right) \longrightarrow H_{Y^{\prime}, \text { rig }}^{n}\left(X^{\prime}\right) \oplus H_{\text {rig }}^{n-2 d}(Y),
\end{gathered}
$$

définie par

$$
\psi_{n}=\left(\begin{array}{ll}
f^{*} & j_{*} \\
0 & g_{*}
\end{array}\right)
$$

Proposition 7.12 (cohomologie d'un éclaté). — Avec les notations ci-dessus, $\psi_{n}\left(\right.$ resp. $\left.\psi_{Y, n}\right)$ est un isomorphisme.

Pour finir, regardons $F: X \rightarrow X$ le morphisme de Frobenius absolu. Il induit

$$
\Phi: H_{i}^{\mathrm{rig}}(X) \longrightarrow H_{i}^{\mathrm{rig}}(X) .
$$

Proposition 7.13. - Soit $Z$ une sous-variété de codimension $r$ dans $X$. On a

$$
\Phi(\gamma(Z))=p^{r} \gamma(Z)
$$

TOME $131-2003-\mathrm{N}^{\mathrm{O}} 1$ 
Démonstration. - Par linéarité, il suffit de démontrer le résultat sur la classe fondamentale d'un variété intègre $X$. Ensuite, on peut supposer de plus que $X$ est lisse grâce au lemmes 6.2 et 6.3 . Dès lors, le résultat découle de la proposition 7.7 et du résultat similaire pour les classes de cycles [15, B.2.2].

\section{BIBLIOGRAPHIE}

[EGAI] Grothendieck (A.) \& Dieudonné (J.A.) - Eléments de géométrie algébrique I, Grundlehren der mathematischen Wissenschaften, vol. 166, Springer-Verlag, 1971.

[EGAIV] Grothendieck (A.) \& Dieudonné (J.A.) - Eléments de géométrie algébrique, Publications Math., vol. 20, 24, 28, 32, IHES, 19641967.

[SGA4] Artin (M.), Grothendieck (A.) \& Verdier (J.L.) - Théorie des topos et cohomologie étale des schémas, Lectures Notes in Math., vol. 269, 270, 305, Springer-Verlag, 1972-1973.

[SGA6] Berthelot (P.), Grothendieck (A.) \& Illusie (L.) - Théorie des intersections et théorème de Riemann-Roch, Lectures Notes in Math., vol. 225, Springer-Verlag, 1971.

[1] Berthelot (P.) - Cohomologie cristalline des schémas de caractéristique $p>0$, Lectures Notes in Math., vol.407, Springer-Verlag, 1974.

[2] Géométrie rigide et cohomologie rigide des variétés algébriques de caractéristique p, Mém. Soc. Math. France, t. 23 (1986), pp. $7-32$.

[3] — Lettre de Berthelot à Illusie, 1990.

[4] Cohomologie rigide et cohomologie rigide à support propre, Prépublications IRMAR, 1996.

[5] — $\mathscr{D}$-modules arithmétiques I. Opérateurs différentiels de niveau fini, Ann. Sci. École Norm. Sup., t. 29 (1996), pp. 185-272.

[6] Dualité de Poincaré et formule de Künneth en cohomologie rigide, C. R. Acad. Sci. Paris, t. 325 (1997), pp. 493-498.

[7] Finitude et pureté cohomologique en cohomologie rigide, Invent. Math., t. 128 (1997), pp. 329-377.

[8] Berthelot (P.) \& Illusie (L.) - Classes de Chern en cohomologie cristalline, C. R. Acad. Sci. Paris, t. 270 (1970), pp. 1695-1697, 17501752 .

[9] Berthelot (P.) \& Ogus (A.) - F-isocrystals and De Rham cohomology I, Invent. Math., t. 72 (1983), pp. 159-199.

[10] Bloch (S.) \& Ogus (A.) - Gersten's conjecture and the homology of schemes, Ann. Sci. École Norm. Sup., t. 7 (1974), pp. 181-202. 
[11] Elkik (R.) - L'équivalence rationnelle, in Séminaire de géométrie analytique (École Norm. Sup., Paris, 1974-75), Astérisque, vol. 3637, Soc. Math. France, Paris, 1976, pp. 35-63.

[12] Fulton (W.) - Rational equivalence on singular varieties, Inst. Hautes Études Sci. Publ. Math., t. 45 (1975), pp. 147-167.

[13] , Intersection theory, Ergebnisse der Mathematik und ihrer Grenzgebiete, Springer-Verlag, 1984.

[14] Gillet (H.) - Riemann-Roch theorems for higher algebraic $K$ theory, Advances in Math., t. 40 (1981), pp. 203-289.

[15] Gillet (H.) \& Messing (W.) - Cycle classes and Riemann-Roch for crystalline cohomology, Duke Math. J., t. 55 (1987), no.3, pp. 501-538.

[16] Gros (M.) - Classes de Chern et classes de cycles en cohomologie de Hodge-Witt logarithmique, Mém. Soc. Math. France, vol. 21, Gauthier-Villars, 1985.

[17] Grosse-KLönne (E.) - Finiteness of de Rham cohomology in rigid analysis, Preprint : to appear in Duke Math. J., 2000.

[18] Grothendieck (A.) - La théorie des classes de Chern, Bull. Soc. Math. France, t. 86 (1958), pp. 137-154.

[19] , Local cohomology, Lecture Notes in Math., vol. 41, SpringerVerlag, 1967.

[20] Classes de Chern et représentations linéaires des groupes discrets, in Dix exposés sur la cohomologie des schémas, North-Holland, 1968, pp. 215-305.

[21] Hartshorne (R.) - On the De Rham cohomology of algebraic varieties, Inst. Hautes Études Sci. Publ. Math., t. 45 (1976), pp. 5-99.

[22] Hirzebruch (F.) - Topological methods in algebraic geometry, Grundlehren der Math. Wissenschaften, vol. 131, Springer-Verlag, 1966.

[23] Illusie (L.) - Complexe cotangent et déformations II, Lecture Notes in Math., vol. 283, Springer-Verlag, 1972.

[24] Jannsen (U.) - Mixed motives and algebraic K-theory, Lecture Notes in Math., vol. 1400, Springer-Verlag, 1989.

[25] LütKeвонmeRT (W.) - Formal-algebraic and rigid-analytic geometry, Math. Ann., t. 286 (1990), pp. 341-371.

[26] Monsky (P.) \& Washnitzer (G.) - Formal cohomology I, Ann. of Math., t. 88 (1968), pp. 218-238.

[27] Nagata (M.) - Embedding of an abstract variety in a complete variety, J. Math. Kyoto Univ., t. 2 (1962), pp. 1-10.

[28] Niziol (W.) - On the image of p-adic regulators, Invent. Math., t. 127 (1997), pp. 375-400.

TOME $131-2003-\mathrm{N}^{\mathrm{O}} 1$ 
[29] Ogus (A.) - The convergent topos in characteristic $p$, in The Grothendieck Festschrifft, North-Holland, 1968, pp. 133-162.

[30] Petrequin (D.) - Classes de Chern et classes de cycle en cohomologie rigide, Thèse, Université Rennes I, 2000.

[31] Classes de cycles en cohomologie rigide, Prépublication IRMAR 01-06, Université Rennes I, 2001.

[32] Raynaud (M.) - Géométrie analytique rigide, d'après Tate, Kiehl,..., in Table Ronde d'Analyse non archimédienne (Paris, 1972), Mém. Soc. Math. France, vol. 39/40, 1974, pp. 319-327.

[33] Raynaud (M.) \& Gruson (L.) - Critères de platitude et de projectivité, Invent. Math., t. 13 (1971), pp. 1-89.

[34] Trihan (F.) - Théorie de Dieudonné cristalline de niveau variable, Thèse Université Rennes I, 1996. 